\title{
Axial-vector form factors of the baryon octet and chiral symmetry
}

\author{
Ulrich Sauerwein $\oplus^{1,2,3}$ Matthias F. M. Lutz ${ }^{1,2}$ and Rob G. E. Timmermans $\oplus^{3}$ \\ ${ }^{1}$ GSI Helmholtzzentrum für Schwerionenforschung GmbH, Planckstraße 1, 64291 Darmstadt, Germany \\ ${ }^{2}$ Technische Universität Darmstadt, D-64289 Darmstadt, Germany \\ ${ }^{3}$ Van Swinderen Institute for Particle Physics and Gravity, University of Groningen, \\ 9747 AG Groningen, The Netherlands
}

(Received 14 May 2021; accepted 6 January 2022; published 7 March 2022)

\begin{abstract}
We consider the axial-vector form factors of the baryon octet in flavor-SU(3) chiral perturbation theory. The baryon octet and decuplet and the pseudoscalar-meson octet are included as explicit degrees of freedom. We explore the use of on-shell meson and baryon masses in the one-loop contributions to the axial-vector form factors and focus on a consistent treatment in terms of chiral power counting. The convergence properties of such an approach are scrutinized. We discuss the potential for comparison to upcoming QCD lattice data.
\end{abstract}

DOI: 10.1103/PhysRevD.105.054005

\section{INTRODUCTION}

The axial-vector form factors of the octet baryons are, next to the baryon masses, important testing grounds for our understanding of the flavor and chiral structure of lowenergy, nonperturbative QCD. Cabibbo's flavor-SU(3) symmetric model $[1,2]$ for neutron $\beta$ decay and the semileptonic hyperon decays was remarkably successful, but nowadays one requires a more fundamental effective field theory description of these processes in the framework of chiral perturbation theory $(\chi \mathrm{PT})$. Unfortunately, previous work within flavor-SU(3) heavy-baryon $\chi \mathrm{PT}$ has shown serious convergence problems of the chiral expansion $[3,4]$. The expected progress in flavor-SU(3) lattice studies motivates us to revisit the axial-vector form factors of the nucleon and hyperons in $\chi \mathrm{PT}$.

In Ref. [5] we investigated the axial-vector form factor of the nucleon in flavor-SU(2) $\chi \mathrm{PT}$ with nucleons and isobars. Calculations of masses and form factors in $\chi \mathrm{PT}$ face the problem that in the chiral expansion terms occur that violate standard power-counting rules that assume $m_{\pi} \sim \Delta \sim$ small momenta, where $\Delta$ is the isobar-nucleon mass difference. We explored the use of on-shell hadron masses in the loop diagrams to improve the convergence of the chiral expansion when the isobars are included. We showed that it was possible in our scheme to renormalize the one-loop amplitudes in terms of subtracted PassarinoVeltman integrals. We performed a successful global fit of

Published by the American Physical Society under the terms of the Creative Commons Attribution 4.0 International license. Further distribution of this work must maintain attribution to the author(s) and the published article's title, journal citation, and DOI. Funded by SCOAP ${ }^{3}$. the LECs (low-energy constants) to the available flavor-SU (2) QCD lattice data for the nucleon and isobar masses and the nucleon axial-vector form factor [6-9]. An interesting prediction of our approach that results from the use of onshell masses in the loops is a nonanalytic behavior [10,11] of the masses and the form factor as a function of the pion mass, which should become prominent for larger lattice volumes than were used so far.

In this work we extend this approach to calculate the axial-vector form factors of the baryon octet in flavor$\mathrm{SU}(3) \chi \mathrm{PT}$. The baryon decuplet is included as an explicit degree of freedom and consistently incorporated via a suitable subtraction and renormalization scheme similar to Ref. [5]. We investigate whether the use of on-shell masses in loop contributions leads to better convergence properties of the flavor-SU(3) chiral expansion. Unfortunately, a full lattice description of all axial-vector form factors in flavor$\mathrm{SU}(3)$ is not available yet. References [12-14] are restricted to the axial-vector form factor of the nucleon. In Ref. [15] first results for axial-vector form factors of the $\Sigma$ and $\Xi$ hyperons are presented and more results, also for strangeness-changing axial-vector currents, are announced.

We have organized our work as follows. In Sec. II we discuss the flavor-SU(3) chiral Lagrangian with the pseudoscalar-meson Goldstone-boson octet, the baryon octet, and the baryon decuplet as degrees of freedom. Section III discusses the axial-vector currents and form factors in QCD, the prime observables covered in our approach. In Sec. IV we calculate the axial-vector form factors at the one-loop level from the chiral Lagrangian. Next, in Sec. V we discuss our power-counting scheme and the implication for renormalization of the one-loop diagrams. In Sec. VI we investigate the convergence properties of our scheme at the physical point of the form factors and in the flavor-SU(3) 
limit. Finally, Sec. VII contains our summary and an outlook for further work. Several appendixes are devoted to definitions of amplitudes, Clebsch-Gordan coefficients and recoupling constants, and kinematical constants.

\section{THE CHIRAL LAGRANGIAN}

The starting point of all calculations in $\chi \mathrm{PT}$ is the effective chiral Lagrangian, which contains fields of all considered particles: the Goldstone-boson octet $\Phi$, the baryon octet $B$, and the baryon decuplet $\Delta_{\mu}$ [16]. The fields are given, respectively, by

$$
\begin{aligned}
& \Phi=\left(\begin{array}{ccc}
\frac{\eta}{\sqrt{3}}+\pi^{0} & \sqrt{2} \pi^{+} & \sqrt{2} K^{+} \\
\sqrt{2} \pi^{-} & \frac{\eta}{\sqrt{3}}-\pi^{0} & \sqrt{2} K^{0} \\
\sqrt{2} K^{-} & \sqrt{2} \bar{K}^{0} & -\frac{2}{\sqrt{3}} \eta
\end{array}\right), \\
& B=\left(\begin{array}{ccc}
\frac{\Lambda}{\sqrt{6}}+\frac{\Sigma^{0}}{\sqrt{2}} & \Sigma^{+} & p \\
\Sigma^{-} & \frac{\Lambda}{\sqrt{6}}-\frac{\Sigma^{0}}{\sqrt{2}} & n \\
-\Xi^{-} & \Xi^{0} & -\sqrt{\frac{2}{3}} \Lambda
\end{array}\right),
\end{aligned}
$$

$$
\begin{aligned}
\mathcal{L}_{\text {kin }} & =-f^{2} \operatorname{tr}\left[U_{\mu} U^{\mu}\right]+\operatorname{tr}[\bar{B}(i \not D-M) B] \\
& -\operatorname{tr}\left[\bar{\Delta}_{\mu} \cdot\left((i \not D-(M+\Delta)) g^{\mu \nu}-i\left(\gamma^{\mu} D^{\nu}+\gamma^{\nu} D^{\mu}\right)+\gamma^{\mu}(i \not D+(M+\Delta)) \gamma^{\nu}\right) \Delta_{\nu}\right] .
\end{aligned}
$$

We introduced the covariant derivative $D_{\mu}$, which is constructed such that it transforms in the same way as the corresponding field by incorporating the chiral connection $\Gamma$. The mass of the baryon octet in the chiral limit is given by $M$ and the mass of the baryon decuplet in the chiral limit by $M+\Delta$. The Goldstone bosons are massless in the chiral limit. The covariant derivative $D_{\mu}$ and the chiral connection $\Gamma_{\mu}$ are given by

$$
\begin{aligned}
D_{\mu} B & =\partial_{\mu} B+\Gamma_{\mu} B-B \Gamma_{\mu}, \\
\left(D_{\mu} \Delta_{\nu}\right)^{a b c} & =\partial_{\mu} \Delta_{\nu}^{a b c}+\Gamma_{\mu, n}^{a} \Delta_{\nu}^{n b c}+\Gamma_{\mu, n}^{b} \Delta_{\nu}^{a n c}+\Gamma_{\mu, n}^{c} \Delta_{\nu}^{a b n}, \\
\Gamma_{\mu} & =\frac{1}{2} u^{\dagger}\left[\partial_{\mu}-i\left(v_{\mu}+a_{\mu}\right)\right] u+\frac{1}{2} u\left[\partial_{\mu}-i\left(v_{\mu}-a_{\mu}\right)\right] u^{\dagger}, \quad u=e^{i \frac{\Phi}{2 f}}, \\
U_{\mu} & =\frac{1}{2} u^{\dagger}\left(\partial_{\mu} e^{i \frac{\Phi}{f}}\right) u^{\dagger}-\frac{i}{2} u^{\dagger}\left(v_{\mu}+a_{\mu}\right) u+\frac{i}{2} u\left(v_{\mu}-a_{\mu}\right) u^{\dagger},
\end{aligned}
$$

and $U_{\mu}$ contains the Goldstone-boson field $\Phi$ and possible external currents with vector $v_{\mu}$ or axial-vector structure $a_{\mu}$. The pion-decay constant in the chiral limit is denoted by $f \simeq 87 \mathrm{MeV}$ [19]. Furthermore, we specify

$$
\mathcal{L}^{(1)}=F \operatorname{tr}\left[\bar{B} \gamma^{\mu} \gamma_{5}\left[i U_{\mu}, B\right]\right]+D \operatorname{tr}\left[\bar{B} \gamma^{\mu} \gamma_{5}\left\{i U_{\mu}, B\right\}\right]+C \operatorname{tr}\left[\left(\bar{\Delta}_{\mu} \cdot i U^{\mu}\right) B+\text { H.c. }\right]+H \operatorname{tr}\left[\left(\bar{\Delta}^{\mu} \cdot \gamma_{5} \gamma_{\nu} \Delta_{\mu}\right) i U^{\nu}\right]
$$

In the lowest-order Lagrangian $\mathcal{L}^{(1)}$ we introduced for the decuplet fields the shorthand notation [16]

$$
\begin{aligned}
\left(\Phi \cdot \Delta_{\mu}\right)_{b}^{a} & =\epsilon_{k l b} \Phi_{n}^{l} \Delta_{\mu}^{k n a}, \quad\left(\bar{\Delta}^{\mu} \cdot \Phi\right)_{b}^{a}=\epsilon^{k l a} \bar{\Delta}_{k n b}^{\mu} \Phi_{l}^{n}, \\
\left(\bar{\Delta}^{\mu} \cdot \Delta_{\mu}\right)_{b}^{a} & =\bar{\Delta}_{b c d}^{\mu} \Delta_{\mu}^{a c d},
\end{aligned}
$$

and the dimensionless LECs $F, D, C$, and $H$. For convenience the Lagrangians $\mathcal{L}^{(2)}$ and $\mathcal{L}^{(3)}$ are split as 


$$
\begin{gathered}
\mathcal{L}^{(2)}=\mathcal{L}^{(S)}+\mathcal{L}^{(V)}+\mathcal{L}^{(T)}+\mathcal{L}^{(A, a)}+\mathcal{L}^{(A, b)}+\mathcal{L}^{(F)}, \\
\mathcal{L}^{(3)}=\mathcal{L}^{(\chi)}+\mathcal{L}^{(R)}
\end{gathered}
$$

with

$$
\begin{aligned}
\mathcal{L}^{(S)}= & -\frac{1}{2} g_{0}^{(S)} \operatorname{tr}[\bar{B} B] \operatorname{tr}\left[U_{\mu} U^{\mu}\right]-\frac{1}{2} g_{1}^{(S)} \operatorname{tr}\left[\bar{B} U^{\mu}\right] \operatorname{tr}\left[U_{\mu} B\right]-\frac{1}{4} g_{F}^{(S)} \operatorname{tr}\left[\bar{B}\left[\left\{U^{\mu}, U_{\mu}\right\}, B\right]\right]-\frac{1}{4} g_{D}^{(S)} \operatorname{tr}\left[\bar{B}\left\{\left\{U^{\mu}, U_{\mu}\right\}, B\right\}\right], \\
\mathcal{L}^{(V)}= & -\frac{1}{4} g_{0}^{(V)} \operatorname{tr}\left[\bar{B} i \gamma^{\mu} D^{\nu} B\right] \operatorname{tr}\left[U_{\nu} U_{\mu}\right] \\
& -\frac{1}{8} g_{1}^{(V)}\left(\operatorname{tr}\left[\bar{B} U_{\mu}\right] i \gamma^{\mu} \operatorname{tr}\left[U_{\nu} D^{\nu} B\right]+\operatorname{tr}\left[\bar{B} U_{\nu}\right] i \gamma^{\mu} \operatorname{tr}\left[U_{\mu} D^{\nu} B\right]\right) \\
& -\frac{1}{8} g_{F}^{(V)} \operatorname{tr}\left[\bar{B} i \gamma^{\mu}\left[\left\{U_{\mu}, U_{\nu}\right\}, D^{\nu} B\right]\right] \\
& -\frac{1}{8} g_{D}^{(V)} \operatorname{tr}\left[\bar{B} i \gamma^{\mu}\left\{\left\{U_{\mu}, U_{\nu}\right\}, D^{\nu} B\right\}\right]+\text { H.c., } \\
\mathcal{L}^{(T)}= & -\frac{1}{2} g_{1}^{(T)} \operatorname{tr}\left[\bar{B} U_{\mu}\right] i \sigma^{\mu \nu} \operatorname{tr}\left[U_{\nu} B\right]-\frac{1}{4} g_{F}^{(T)} \operatorname{tr}\left[\bar{B} i \sigma^{\mu \nu}\left[\left[U_{\mu}, U_{\nu}\right], B\right]\right]-\frac{1}{4} g_{D}^{(T)} \operatorname{tr}\left[\bar{B} i \sigma^{\mu \nu}\left\{\left[U_{\mu}, U_{\nu}\right], B\right\}\right] \\
\mathcal{L}^{(A, a)}= & -\frac{1}{4} f_{1}^{(A)} \operatorname{tr}\left[\left(\bar{\Delta}^{\mu} \cdot \gamma^{\nu} \gamma_{5} B\right)\left\{U_{\mu}, U_{\nu}\right\}\right]-\frac{1}{4} f_{3}^{(A)} \operatorname{tr}\left[\left(\bar{\Delta}^{\mu} \cdot U_{\nu}\right) \gamma^{\nu} \gamma_{5} U_{\mu} B+\left(\bar{\Delta}^{\mu} \cdot U_{\mu}\right) \gamma^{\nu} \gamma_{5} U_{\nu} B\right]+\text { H.c. }, \\
\mathcal{L}^{(A, b)}= & -\frac{1}{4} f_{2}^{(A)} \operatorname{tr}\left[\left(\bar{\Delta}^{\mu} \cdot \gamma^{\nu} \gamma_{5} B\right)\left[U_{\mu}, U_{\nu}\right]\right]-\frac{1}{4} f_{4}^{(A)} \operatorname{tr}\left[\left(\bar{\Delta}^{\mu} \cdot U_{\nu}\right) \gamma^{\nu} \gamma_{5} U_{\mu} B-\left(\bar{\Delta}^{\mu} \cdot U_{\mu}\right) \gamma^{\nu} \gamma_{5} U_{\nu} B\right]+\text { H.c., } \\
\mathcal{L}^{(F)=} & g_{F}^{(F)} \operatorname{tr}\left[\bar{B}\left[F_{\mu \nu}^{+}, \sigma^{\mu \nu} B\right]\right]+g_{D}^{(F)} \operatorname{tr}\left[\bar{B}\left\{F_{\mu \nu}^{+}, \sigma^{\mu \nu} B\right\}\right]+f_{M}^{(F)} \operatorname{tr}\left[\bar{B} \gamma^{\mu} \gamma_{5}\left(i F_{\mu \nu}^{+} \cdot \Delta^{\nu}\right)+\text { H.c. }\right]-f_{E}^{(F)} \operatorname{tr}\left[\bar{B} \gamma^{\mu}\left(i F_{\mu \nu}^{-} \cdot \Delta^{\nu}\right)+\text { H.c. }\right] .
\end{aligned}
$$

Furthermore we specify

$$
\begin{aligned}
\mathcal{L}^{(\chi)}= & -\frac{1}{2} g_{1}^{(\chi)} \operatorname{tr}\left[\bar{B} \gamma_{5} \gamma^{\mu}\left\{i U_{\mu},\left\{\chi_{0}, B\right\}\right\}+\text { H.c. }\right]-\frac{1}{2} g_{2}^{(\chi)} \operatorname{tr}\left[\bar{B} \gamma_{5} \gamma^{\mu}\left\{i U_{\mu},\left[\chi_{0}, B\right]\right\}+\text { H.c. }\right] \\
- & \frac{1}{2} g_{3}^{(\chi)} \operatorname{tr}\left[\bar{B} \gamma_{5} \gamma^{\mu}\left[i U_{\mu},\left\{\chi_{0}, B\right\}\right]+\text { H.c. }\right]-\frac{1}{2} g_{4}^{(\chi)} \operatorname{tr}\left[\bar{B} \gamma_{5} \gamma^{\mu}\left[i U_{\mu},\left[\chi_{0}, B\right]\right]+\text { H.c. }\right] \\
& -\frac{1}{2} g_{5}^{(\alpha)} \operatorname{tr}\left[\bar{B} \gamma_{5} \gamma^{\mu} B \operatorname{tr}\left[\chi_{0} i U_{\mu}\right]+\text { H.c. }\right]-\frac{1}{2} g_{6}^{(\chi)} \operatorname{tr}\left[\bar{B} \gamma_{5} \gamma^{\mu} i U_{\mu} \operatorname{tr}\left[\chi_{0} B\right]+\text { H.c. }\right]-g_{7}^{(\chi)} \operatorname{tr}\left[\bar{B} \gamma_{5} \gamma^{\mu}\left[i U_{\mu}, B\right]\right] \operatorname{tr}\left[\chi_{0}\right],
\end{aligned}
$$

and

$$
\begin{aligned}
\mathcal{L}^{(R)}= & g_{F}^{(R)} \operatorname{tr}\left[\bar{B} \gamma^{\mu} \gamma_{5}\left[\left[D^{\nu}, F_{\mu \nu}^{-}\right], B\right]\right] \\
& +g_{D}^{(R)} \operatorname{tr}\left[\bar{B} \gamma^{\mu} \gamma_{5}\left\{\left[D^{\nu}, F_{\mu \nu}^{-}\right], B\right\}\right]
\end{aligned}
$$

Here two new operators appear, $\chi_{0}$ and $F_{\mu \nu}^{ \pm}$, where

$$
\chi_{0}=2 B_{0}\left(\begin{array}{ccc}
m & 0 & 0 \\
0 & m & 0 \\
0 & 0 & m_{s}
\end{array}\right) .
$$

In order to implement explicit chiral symmetry breaking, $\chi_{0}$ introduces the finite quark masses $m$ and $m_{s}$, where we assume the strict isospin limit $m_{u}=m_{d} \equiv m$. Further- more, a derivative acting on the external axial-vector current $a_{\mu}$ is needed,

$$
F_{\mu \nu}^{ \pm}=u^{\dagger} F_{\mu \nu}^{R} u \pm u F_{\mu \nu}^{L} u^{\dagger}
$$

with

$$
\begin{gathered}
F_{\mu \nu}^{R}=\partial_{\mu} a_{\nu}-\partial_{\nu} a_{\mu}-i\left[a_{\mu}, a_{\nu}\right], \\
F_{\mu \nu}^{L}=-\left(\partial_{\mu} a_{\nu}-\partial_{\nu} a_{\mu}\right)-i\left[a_{\mu}, a_{\nu}\right] .
\end{gathered}
$$

All coefficients $g_{(. .)}^{(\cdots)}$ and $f_{(\cdots)}^{(\cdots)}$ are a priori unknown LECs. Three LECs in Ref. [16], corresponding to $\mathcal{L}^{(\chi)}$, are redundant. In Table I we link our Lagrangian $\mathcal{L}$ to other conventions found in the literature. Terms including 
TABLE I. Link of the LECs, used in this work, to previous publications [18,21,22].

\begin{tabular}{lccc}
\hline \hline This work & {$[18,21,22]$} & This work & {$[18,21,22]$} \\
\hline$D$ & $D$ & $g_{0}^{(S)}$ & $8\left(-b_{1}+b_{2}+b_{4}\right)$ \\
$F$ & $F$ & $g_{1}^{(S)}$ & $16\left(-b_{1}+b_{2}\right)$ \\
$C$ & $\frac{1}{\sqrt{2}} h_{A}$ & $g_{F}^{(S)}$ & $8 b_{3}$ \\
$H$ & $H_{A}$ & $g_{D}^{(S)}$ & $8\left(3 b_{1}-b_{2}\right)$ \\
$g_{0}^{(V)}$ & $16\left(-b_{5}+b_{7}+b_{8}\right)$ & $g_{1}^{(T)}$ & $8 d_{3}$ \\
$g_{1}^{(V)}$ & $32\left(-b_{5}+b_{7}\right)$ & $g_{F}^{(T)}$ & $16 d_{2}$ \\
$g_{F}^{(V)}$ & $16 b_{6}$ & $g_{D}^{(T)}$ & $16 d_{1}$ \\
$g_{D}^{(V)}$ & $16\left(3 b_{5}-b_{7}\right)$ & & \\
$g_{F}^{(F)}$ & $b_{M, F}$ & $f_{1}^{(A)}$ & $\frac{4}{5} c_{(27)}-4 c_{D}$ \\
$g_{D}^{(F)}$ & $b_{M, D}$ & $f_{2}^{(A)}$ & $-\frac{8}{3} c_{(10)}-4 c_{F}$ \\
$f_{M}^{(F)}$ & $c_{M}$ & $f_{3}^{(A)}$ & $4 c_{(27)}$ \\
$f_{E}^{(F)}$ & $c_{E}$ & $f_{4}^{(A)}$ & $-8 c_{(10)}$ \\
$g_{F}^{(R)}$ & $d_{64}$ & $g_{D}^{(R)}$ & $d_{65}$ \\
$g_{1}^{(\chi)}$ & $2\left(4 d_{44}+3 d_{46}\right)$ & $g_{5}^{(\chi)}$ & $4\left(-d_{46}+d_{47}\right)$ \\
$g_{2}^{(\chi)}$ & $8 d_{43}$ & $g_{6}^{(\chi)}$ & $-8 d_{46}$ \\
$g_{3}^{(\chi)}$ & $8 d_{42}$ & $g_{7}^{(\chi)}$ & $4 d_{45}$ \\
$g_{4}^{(\chi)}$ & $2\left(4 d_{41}+d_{46}\right)$ & & \\
\hline \hline
\end{tabular}

exclusively the baryon octet have been derived in Refs. [18,20,21], whereas the terms also including the decuplet are compared to Ref. [22]. Our LECs $g_{(\cdots)}^{(\cdots)}$ and $f_{(\cdots)}^{(\cdots)}$ are normalized in such a way that we expect their values to be in the range $\sim[-10,10]$. References $[18,21,22]$ use in part a different normalization. The comparison between our LECs $f_{1,2,3,4}^{(A)}$ and the LECs $c_{F, D,(10),(27)}$, performed in Ref. [22], agrees with our translation in Table I.

\section{AXIAL-VECTOR CURRENTS}

\section{A. Motivation}

The matrix element $\mathcal{M}$ of the semileptonic decay process of a nucleon or hyperon $\left(b \rightarrow \bar{b}+e^{-}+\bar{\nu}\right)$ can be parametrized [2] as

$$
\begin{aligned}
& \mathcal{M}=\frac{G_{F} V_{u d / s}}{\sqrt{2}} \bar{u}_{\bar{b}}\left(O_{\alpha}^{V}+O_{\alpha}^{A}\right) u_{b} \bar{u}_{e} \gamma^{\alpha}\left(1+\gamma_{5}\right) v_{\nu}, \\
& O_{\alpha}^{V}=f_{1}^{(\bar{b} b)}\left(q^{2}\right) \gamma_{\alpha}+\frac{q^{\beta}}{M_{B}} f_{2}^{(\bar{b} b)}\left(q^{2}\right) \sigma_{\alpha \beta}+\frac{q_{\alpha}}{M_{B}} f_{3}^{(\bar{b} b)}\left(q^{2}\right), \\
& O_{\alpha}^{A}=g_{1}^{(\bar{b} b)}\left(q^{2}\right) \gamma_{\alpha} \gamma_{5}+\frac{q^{\beta}}{M_{B}} g_{2}^{(\bar{b} b)}\left(q^{2}\right) \sigma_{\alpha \beta} \gamma_{5}+\frac{q_{\alpha}}{M_{B}} g_{3}^{(\bar{b} b)}\left(q^{2}\right) \gamma_{5},
\end{aligned}
$$

with the Fermi constant $G_{F}$ and the Cabibbo-KobayashiMaskawa (CKM) matrix elements $V_{u d} \simeq 0.97$ for
TABLE II. Cabibbo theory for the form factor $g_{1}^{(\bar{b} b)}(0)$ [2]. We use $F=0.45$ and $D=0.80$ [23] for the numerical estimate.

\begin{tabular}{lcccc}
\hline \hline $\begin{array}{l}\text { Process } \\
b \rightarrow \bar{b}\end{array}$ & CKM & $g_{1}^{(\bar{b} b)}(0)$ & Estimate & $\begin{array}{c}\text { Experiment } \\
{[24]}\end{array}$ \\
\hline$n \rightarrow p e^{-} \bar{\nu}$ & $V_{u d}$ & $D+F$ & 1.25 & $1.2724 \pm 0.0023$ \\
$\Xi^{-} \rightarrow \Xi^{0} e^{-} \bar{\nu}$ & $V_{u d}$ & $D-F$ & 0.35 & $\ldots$ \\
$\Sigma^{-} \rightarrow \Lambda e^{-} \bar{\nu}$ & $V_{u d}$ & $\sqrt{\frac{2}{3}} D$ & 0.65 & $0.601 \pm 0.015[16]$ \\
$\Sigma^{-} \rightarrow \Sigma^{0} e^{-} \bar{\nu}$ & $V_{u d}$ & $\sqrt{2} F$ & 0.64 & $\ldots$ \\
$\Xi^{0} \rightarrow \Sigma^{+} e^{-} \bar{\nu}$ & $V_{u s}$ & $D+F$ & 1.25 & $1.22 \pm 0.05$ \\
$\Sigma^{-} \rightarrow n e^{-} \bar{\nu}$ & $V_{u s}$ & $D-F$ & 0.35 & $0.340 \pm 0.017$ \\
$\Lambda \rightarrow p e^{-} \bar{\nu}$ & $V_{u s}$ & $-\frac{1}{\sqrt{6}}(D+3 F)$ & -0.88 & $-0.879 \pm 0.018$ \\
$\Xi^{-} \rightarrow \Lambda e^{-} \bar{\nu}$ & $V_{u s}$ & $-\frac{1}{\sqrt{6}}(D-3 F)$ & 0.22 & $0.31 \pm 0.06$ \\
$n \rightarrow n$ & $\ldots$ & $-\frac{1}{\sqrt{6}}(D-3 F)$ & 0.22 & $\ldots$ \\
$\Sigma^{-} \rightarrow \Sigma^{-}$ & $\ldots$ & $\sqrt{\frac{2}{3}} D$ & 0.65 & $\ldots$ \\
$\Lambda \rightarrow \Lambda$ & $\ldots$ & $-\sqrt{\frac{2}{3}} D$ & -0.65 & $\ldots$ \\
$\Xi^{-} \rightarrow \Xi^{-}$ & $\ldots$ & $-\frac{1}{\sqrt{6}}(D+3 F)$ & -0.88 & $\ldots$ \\
\hline \hline
\end{tabular}

strangeness-conserving and $V_{u s} \simeq 0.22$ for strangenesschanging decays [19]. The form factors $g_{1,2,3}^{(\bar{b} b)}\left(q^{2}\right)$ and $f_{1,2,3}^{(\bar{b} b)}\left(q^{2}\right)$ depend on the momentum transfer $q^{2}$. We focus on the axial-vector form factor $g_{1}^{\bar{b} b}\left(q^{2}\right)$. Based on grouptheoretical considerations and assuming exact flavor-SU(3) symmetry, the Cabibbo model is able to describe $g_{1}^{(\bar{b} b)}\left(q^{2}\right)$ of 12 processes with only two reduced matrix elements. These refer, translated to our framework, to the LECs $D$ and $F$ from the chiral Lagrangian $\mathcal{L}^{(1)}$ from Eq. (7). We show Cabibbo's results for $g_{1}^{\bar{b} b}\left(q^{2}\right)$ in terms of $D$ and $F$ in Table II.

Roughly ten years ago first results from nonperturbative calculations of axial-vector form factors on the lattice became available (e.g. Refs. [25,26]). The quark mass is an input parameter in these kinds of calculations. Therefore additional data points can be produced, also in unphysical regions [12-15]. Since for larger quark masses flavorSU(3)-breaking effects play an important role, the Cabibbo model is not suitable for more detailed investigations. $\chi \mathrm{PT}$ offers the opportunity to consistently add higher-order contributions, such as finite quark masses. Therefore we scrutinize axial-vector form factors in $\chi \mathrm{PT}$ to one-loop order and derive their dependence on the LECs of the chiral Lagrangian of Eq. (4).

\section{B. Definition of axial-vector form factors}

In QCD the axial-vector currents take the form [27]

$$
A_{i}^{\mu}(x)=\bar{q}(x) \gamma^{\mu} \gamma_{5} \frac{\lambda_{i}}{2} q(x)
$$

with the three-component quark field $q(x)$ and the GellMann matrices $\lambda_{i}$ for flavor-SU(3). The axial-vector 
TABLE III. Baryon-octet multiplets $B$.

\begin{tabular}{lcc}
\hline \hline Singlet & Doublets & Triplet \\
\hline$\Lambda=\left(\Lambda^{0}\right) \quad N=\left(\begin{array}{c}p \\
n\end{array}\right) \quad \Xi=\left(\begin{array}{c}\Xi^{0} \\
\Xi^{-}\end{array}\right) \quad \Sigma=\left(\begin{array}{c}\frac{\Sigma^{+}+\Sigma^{-}}{\sqrt{2}} \\
\frac{i \Sigma^{+}-i \Sigma^{-}}{\sqrt{2}} \\
\Sigma^{0}\end{array}\right)$ \\
\hline \hline
\end{tabular}

couplings can be incorporated as external fields $a_{\mu}(x)=$ $\sum_{i} a_{\mu}^{i}(x) \frac{\lambda_{i}}{2}$ in the QCD Lagrangian. They enter the chiral Lagrangian as a part of $U_{\mu}$ in Eq. (6).

We consider an axial-vector current $A_{i}^{\mu}(x=0)$ coupling to an incoming baryon $B$ (with mass $M_{p}$ and momentum $p$ ) and an outgoing baryon $\bar{B}$ (with mass $M_{\bar{p}}$ and momentum $\bar{p}$ ). In contrast to the baryons $\bar{b}$ and $b$ in the previous section, we present the baryons here in isospin multiplets $\bar{B}$ and $B$, shown in Table III. Isospin-breaking effects are neglected. We restrict the external baryon fields to the octet. The matrix element for the axial-vector current can be decomposed into three parts as

$$
\begin{aligned}
\left\langle\bar{B}(\bar{p})\left|A_{i}^{\mu}(0)\right| B(p)\right\rangle & \\
= & \bar{u}_{\bar{B}}(\bar{p})\left(\gamma^{\mu} G_{A, i}^{(\bar{B} B)}\left(q^{2}\right)+\frac{q^{\mu}}{M_{\bar{p}}+M_{p}} G_{P, i}^{(\bar{B} B)}\left(q^{2}\right)\right. \\
& \left.+\frac{(\not p+\not p)\left(\bar{p}^{\mu}+p^{\mu}\right)}{\left(M_{\bar{p}}+M_{p}\right)^{2}} G_{I, i}^{(\bar{B} B)}\left(q^{2}\right)\right) \gamma_{5} T_{I(\bar{B}) I(B)}^{(i)} u_{B}(p),
\end{aligned}
$$

with $q^{\mu}=(\bar{p}-p)^{\mu}$ and the index $i=1, \ldots, 8$ running over the different types of strangeness-conserving $(\Delta S=0)$ and strangeness-changing $(\Delta S= \pm 1)$ axial-vector currents. The coefficients $G_{A, i}^{(\bar{B} B)}\left(q^{2}\right)$ and $G_{P, i}^{(\bar{B} B)}\left(q^{2}\right)$ are known as the axial-vector and pseudoscalar form factors. The third form factor $G_{I, i}^{(\bar{B} B)}\left(q^{2}\right)$ is, by construction, only nonzero for processes with $\bar{B} \neq B$ for some specific Feynman diagrams.

Strict isospin symmetry forces the axial-vector form factor $G_{A, i}^{(B B)}\left(q^{2}\right)$ to be equal within isospin multiplets. We express the form factor in the isospin basis and use the isospin equivalent axial-vector current $a_{\mu}^{X}$ with $X \in\{\pi, K, \bar{K}, \eta\}$, i.e.

$G_{A, 1}^{(\bar{B} B)}=G_{A, 2}^{(\bar{B} B)}=G_{A, 3}^{(\bar{B} B)} \rightarrow G_{A, a_{\mu}^{n}}^{(\bar{B} B)}, \quad G_{A, 8}^{(\bar{B} B)} \rightarrow G_{A, a_{\mu}^{n}}^{(\bar{B} B)}$,

$G_{A, 4}^{(\bar{B} B)}=G_{A, 5}^{(\bar{B} B)} \rightarrow G_{A, a_{\mu}^{K}}^{(\bar{B} B)}, \quad G_{A, 6}^{(\bar{B} B)}=G_{A, 7}^{(\bar{B} B)} \rightarrow G_{A, a_{\mu}^{\bar{K}}}^{(\bar{B} B)}$.

The isospin transition matrices $T_{I(\bar{B}) I(B)}^{(i)}$ depend on the isospin of the outgoing/incoming baryon $I(\bar{B}) / I(B)$ and the isospin of the axial-vector current $I(i)$,

$$
T_{I(\bar{B}) I(B)}^{(i)}= \begin{cases}T_{I(\bar{B}) I(B), i}^{(1)} & \text { if } i=1,2,3, \\ T_{I(\bar{B}) I(B), i-3}^{(1 / 2)} & \text { if } i=4,5, \\ \tilde{T}_{I(\bar{B}) I(B), i-5}^{(1 / 2)} & \text { if } i=6,7, \\ T_{I(\bar{B}) I(B), i-7}^{(0)} & \text { if } i=8 .\end{cases}
$$

An isospin transition matrix $T_{I(\bar{B}) I(B), i}^{I(i)}$ has $2 I(i)+1$ components, each of them being a $(2 I(\bar{B})+1) \times(2 I(B)+1)$ matrix. Specifically,

$$
\begin{aligned}
& \left(T_{00,1}^{(0)}\right)=\frac{1}{\sqrt{2}}, \quad\left(T_{\frac{11}{22}, 1}^{(0)}\right)_{j k}=\frac{1}{\sqrt{2}}(\delta)_{j k}, \quad\left(T_{11,1}^{(0)}\right)_{j k}=\frac{1}{\sqrt{2}}(\delta)_{j k}, \\
& \left(T_{11, i}^{(1)}\right)_{j k}=-\frac{i}{\sqrt{2}} \epsilon_{i j k}, \quad\left(T_{01, i}^{(1)}\right)_{j}=\left(T_{10, i}^{(1)}\right)_{j}=\frac{1}{\sqrt{2}}\left(\delta^{i}\right)_{j}, \quad\left(T_{\frac{11}{22}, i}^{(1)}\right)_{j k}=(-1)^{m_{1 / 2}} \frac{1}{2}\left(\tau^{i}\right)_{j k}, \\
& \left(T_{\frac{1}{2} 1, i}^{(1 / 2)}\right)_{j k}=\frac{1}{2}\left(\tau^{k}\right)_{j i}, \quad\left(T_{\frac{1}{2} 0, i}^{(1 / 2)}\right)_{j}=\frac{1}{\sqrt{2}}\left(\delta^{i}\right)_{j}, \quad\left(T_{1 \frac{1}{2}, i}^{(1 / 2)}\right)_{j k}=\frac{i}{2}\left(\tau_{2}\right)_{i l}\left(\tau^{j}\right)_{l k}, \quad\left(T_{0 \frac{1}{2}, i}^{(1 / 2)}\right)_{j}=-\frac{i}{\sqrt{2}}\left(\tau_{2}\right)_{i j}, \\
& \left(\tilde{T}_{1 \frac{1}{2}, i}^{(1 / 2)}\right)_{j k}=\frac{1}{2}\left(\tau^{j}\right)_{k i}, \quad\left(\tilde{T}_{0 \frac{1}{2}, i}^{(1 / 2)}\right)_{j}=\frac{1}{\sqrt{2}}\left(\delta^{i}\right)_{j}, \quad\left(\tilde{T}_{\frac{1}{2}, i}^{(1 / 2)}\right)_{j k}=-\frac{i}{2}\left(\tau^{k}\right)_{j l}\left(\tau_{2}\right)_{l i}, \quad\left(\tilde{T}_{\frac{1}{2}, i}^{(1 / 2)}\right)_{j}=\frac{i}{\sqrt{2}}\left(\tau_{2}\right)_{i j},
\end{aligned}
$$

with $m_{1 / 2}=1$ for $\bar{B}=B=\Xi$ and $m_{1 / 2}=0$ for $\bar{B}=B=N$.

The transition matrices are normalized in such a way that we recover the results of the Cabibbo model at tree level,

$$
G_{A, i \neq 8}^{(\bar{B} B)}\left(q^{2}\right)=g_{1}^{(\bar{b} b)}\left(q^{2}\right) .
$$

We work in the isospin basis with the external baryons $\bar{B}$ and $B$, whereas the form factors in Cabibbo's model are given in particle basis with $\bar{b}$ and $b$. The singlet form factors $G_{A, i=8}^{(\bar{B} B)}\left(q^{2}\right)$ are not related to physical decays.

We will determine the form factors $G_{A, i}^{(\overrightarrow{B B})}\left(q^{2}\right)$ to oneloop level. Divergent loop integrals need to be treated carefully. The technique of dimensional regularization $[28,29]$ introduces the renormalization scale $\mu$, which allows us to determine the integrals in $d$ spacetime dimensions. Subtracting poles of the form $1 /(d-4)$ leads to a finite result, which only depends logarithmically on $\mu$. 
The dimensionful renormalization scale $\mu$ does not interfere with the chiral power counting. Nevertheless, the existence of four different scales, namely the Goldstone-boson mass $m_{\mathrm{GB}}$, the baryon octet(decuplet) mass in the chiral limit $M(M+\Delta)$, and the breakdown scale $\Lambda_{\chi \mathrm{PT}}$, requires consistent power counting. There are different ways to achieve this. Jenkins and Manohar [3] introduced a scheme which uses an expansion in inverse baryon masses (heavy-baryon formalism). The method of infrared regularization was applied by Becher and Leutwyler [30]. The so-called smallscale expansion [31] uses $\Delta$, the decuplet-octet mass difference in the chiral limit, as an expansion parameter. In this work we will follow the strategy of Refs. [5,32], where suitable subtractions assure a consistent power counting, but no expansion in $\Delta$ is performed. The details of our power-counting scheme are given in Chap. V.

\section{DETERMINATION OF AXIAL-VECTOR FORM FACTORS}

\section{A. Analytical results}

In this section we investigate all axial-vector form factors $G_{A, i}^{\bar{B} B}(t)$, with $t=q^{2}$, to $\mathrm{N}^{2} \mathrm{LO}$. We use the baryon multiplets (see Table III) and the axial-vector currents $i=\left\{a_{\mu}^{\pi}, a_{\mu}^{K}\right.$, $\left.a_{\mu}^{\bar{K}}, a_{\mu}^{\eta}\right\}$ in the isospin basis. A typical one-loop diagram is

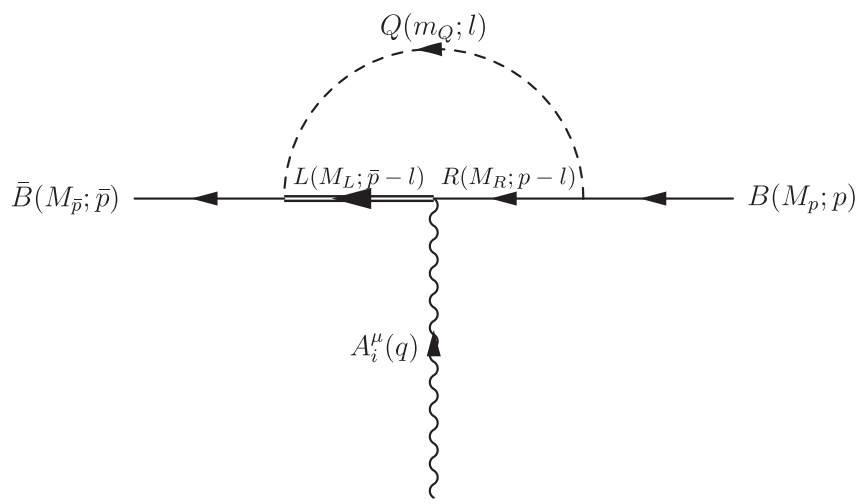

FIG. 1. Example of a one-loop diagram contributing to $G_{A, i}^{\bar{B} B}(t)$. The Goldstone boson $Q$ is represented by a dashed line, whereas the wiggly line is associated with the axial-vector current $A_{i}^{\mu}$. Baryons $(\bar{B}, L, R, B)$ are shown with straight lines, a single line is connected to a octet particle and a double line represents a decuplet particle. We use the notation "label (mass; momentum)".

shown in Fig. 1. In addition to the tree-level diagram, there are three different types of one-loop diagrams contributing to $G_{A, i}^{\bar{B} B}(t)$ : tadpole (Table IV), bubble (Table V), and triangle diagrams (Table VI). All these contributions can be summarized in one condensed formula:

$$
\begin{aligned}
G_{A, i}^{\bar{B} B}(t)= & \sqrt{Z_{\bar{B}} Z_{B}} J^{A} C_{i}^{(\bar{B} B)}+t J^{A} T_{i}^{(\bar{B} B)}+2 B_{0} J^{A} X_{i}^{(\bar{B} B)}+\sum_{Q \in[8]} J_{Q}^{A} \frac{C_{Q, i}^{(\bar{B} B)}}{(2 f)^{2}}+\sum_{n=1}^{5} \sum_{Q \in[8]} \sum_{L \in[8],[10]} J_{L Q}^{A, n} \frac{G_{Q L}^{(\bar{B})}}{2 f} \frac{C_{L Q, i}^{(\bar{B} B), n}}{2 f} \\
& +\sum_{n=1}^{5} \sum_{Q \in[8]} \sum_{R \in[8],[10]} J_{Q R}^{A, n} \frac{C_{Q R, i}^{(\bar{B} B), n}}{2 f} \frac{G_{Q R}^{(B)}}{2 f}+\sum_{n=1}^{2} \sum_{Q \in[8]} \sum_{L, R \in[8],[10]} J_{L Q R}^{A, n} \frac{G_{Q L}^{(\bar{B})}}{2 f} C_{L Q R, i}^{(\bar{B} B), n} \frac{G_{Q R}^{(B)}}{2 f},
\end{aligned}
$$

where we separate the flavor-independent part $J_{\ldots}^{A}$ from the Clebsch-Gordan coefficients (CGCs) $C, T, X$, and $G$, which carry the full flavor information. Sums over the multiplets [8] and [10] of the internal particles $L, R$, and $Q$ (see Fig. 1) indicate loop contributions. The parameter $n$ distinguishes bubble diagrams with contributions from different vertices (see Table V). We also introduced the wave-function renormalization $\sqrt{Z_{\bar{B}} Z_{B}}[3,33]$ modifying the contribution of the tree-level diagram.

The expressions $J_{\ldots}^{A}$ in Eq. (29) represent the axial-vector component of the amplitudes $J^{\mu} \ldots$ of all considered Feynman diagrams. They are given by

\begin{tabular}{|c|c|c|c|c|c|}
\hline Type & Feynman diagram & Projection & Amplitude $J^{\mu} \ldots$ & CGCs & Lagrangian $\mathcal{L}$ \\
\hline Tree & & $J^{A}$ & (A6) & $C_{i}^{(\bar{B} B)}$ & $\mathcal{L}^{(1)}$ \\
\hline Counterterms & & $J^{A}$ & $\begin{array}{l}\text { (A6), } \\
\text { (A7) }\end{array}$ & $\begin{array}{c}X_{i}^{(\bar{B} B)}, \\
T_{i}^{(\bar{B} B)}\end{array}$ & $\begin{array}{l}\mathcal{L}^{(\chi)}, \\
\mathcal{L}^{(R)}\end{array}$ \\
\hline Tadpole & & $J_{Q}^{A}$ & (A8) & $C_{Q, i}^{(\bar{B} B)}$ & $\mathcal{L}^{(1)}$ \\
\hline
\end{tabular}

TABLE IV. Contribution of all tree-level and tadpole diagrams entering Eq. (29). The meaning of different lines is explained in Fig. 1. 
TABLE V. Contribution of all bubble diagrams entering Eq. (29). The meaning of different lines is explained in Fig. 1. The parameter $n$ specifies the origin of the 4-point vertices in bubble diagrams.

\begin{tabular}{|c|c|c|c|c|}
\hline Type & Feynman Diagram & Projection & Amplitude $J_{\ldots}^{\mu}$ & Lagrangian $\mathcal{L}$ \\
\hline Bubble $L \in[8]$ & & $J_{L Q}^{A, n}$ & $\begin{array}{l}\text { (A9), } \\
\text { (A10), } \\
\text { (A11), } \\
\text { (A12), } \\
\text { (A13) }\end{array}$ & $\begin{array}{l}n=1 \leftrightarrow \mathcal{L}_{\mathrm{kin}}, \\
n=2 \leftrightarrow \mathcal{L}^{(S)} \\
n=3 \leftrightarrow \mathcal{L}^{(V)} \\
n=4 \leftrightarrow \mathcal{L}^{(T)} \\
n=5 \leftrightarrow \mathcal{L}^{(F)}\end{array}$ \\
\hline Bubble $R \in[8]$ & & $J_{Q R}^{A, n}$ & $\begin{array}{l}\text { (A14), } \\
\text { (A15), } \\
\text { (A16), } \\
\text { (A17), } \\
\text { (A18) }\end{array}$ & $\begin{array}{l}n=1 \leftrightarrow \mathcal{L}_{\text {kin }}, \\
n=2 \leftrightarrow \mathcal{L}^{(S)} \\
n=3 \leftrightarrow \mathcal{L}^{(V)} \\
n=4 \leftrightarrow \mathcal{L}^{(T)} \\
n=5 \leftrightarrow \mathcal{L}^{(F)}\end{array}$ \\
\hline Bubble $L \in[10]$ & & $J_{L Q}^{A, n}$ & $\begin{array}{l}\text { (A19), } \\
(\mathrm{A} 20), \\
(\mathrm{A} 21)\end{array}$ & $\begin{array}{l}n=1 \leftrightarrow \mathcal{L}^{(A, a)} \\
n=2 \leftrightarrow \mathcal{L}^{(A, b)} \\
n=3 \leftrightarrow \mathcal{L}^{(F)}\end{array}$ \\
\hline Bubble $R \in[10]$ & & $J_{Q R}^{A, n}$ & $\begin{array}{l}(\mathrm{A} 23), \\
(\mathrm{A} 24), \\
(\mathrm{A} 25)\end{array}$ & $\begin{array}{l}n=1 \leftrightarrow \mathcal{L}^{(A, a)} \\
n=2 \leftrightarrow \mathcal{L}^{(A, b)} \\
n=3 \leftrightarrow \mathcal{L}^{(F)}\end{array}$ \\
\hline
\end{tabular}

$$
\begin{aligned}
J_{\ldots}^{A}= & -\frac{\sqrt{2} M_{\bar{p}} M_{p}}{(d-2)\left(M_{\bar{p}} M_{p}+\bar{p} \cdot p\right)} \operatorname{tr}\left[\gamma_{\mu} \gamma_{5} \frac{\bar{p}+M_{\bar{p}}}{2 M_{\bar{p}}} J \ldots \frac{\not p+M_{p}}{2 M_{p}}\right] \\
& -\frac{M_{\bar{p}} M_{p}\left(M_{\bar{p}}+M_{p}\right)^{2}}{\sqrt{2}(d-2)\left(M_{\bar{p}} M_{p}-\bar{p} \cdot p\right)\left(M_{\bar{p}} M_{p}+\bar{p} \cdot p\right)} \operatorname{tr}\left[\frac{\bar{p}_{\mu}-p_{\mu}}{M_{\bar{p}}+M_{p}} \gamma_{5} \frac{\bar{p}+M_{\bar{p}}}{2 M_{\bar{p}}} J_{\ldots}^{\mu} \frac{\not p+M_{p}}{2 M_{p}}\right] \\
& -\frac{M_{\bar{p}} M_{p}\left(M_{\bar{p}}+M_{p}\right)^{2}}{\sqrt{2}(d-2)\left(M_{\bar{p}} M_{p}-\bar{p} \cdot p\right)\left(M_{\bar{p}} M_{p}+\bar{p} \cdot p\right)} \operatorname{tr}\left[\frac{(\not p+\not p)\left(\bar{p}_{\mu}+p_{\mu}\right)}{\left(M_{\bar{p}}+M_{p}\right)^{2}} \gamma_{5} \frac{\not p}{2 M_{\bar{p}}} J_{\overline{\bar{p}}}^{\mu} \frac{\not p+M_{p}}{2 M_{p}}\right],
\end{aligned}
$$

\begin{tabular}{|c|c|c|c|}
\hline Type & Diagram & Projection & $\begin{array}{c}\text { Amplitude } \\
J_{\ldots}^{\mu}\end{array}$ \\
\hline $\begin{array}{l}\text { Triangle } \\
L \in[8]\end{array}$ & & $J_{L Q R}^{A, 1}$ & (A27) \\
\hline$R \in[8]$ & & & \\
\hline $\begin{array}{l}\text { Triangle } \\
L \in[10]\end{array}$ & & $J_{L Q R}^{A, 1,2}$ & (A28), (A31) \\
\hline$R \in[8]$ & & & \\
\hline $\begin{array}{l}\text { Triangle } \\
L \in[8]\end{array}$ & & $J_{L Q R}^{A, 1,2}$ & (A29), (A32) \\
\hline$R \in[10]$ & & & \\
\hline $\begin{array}{l}\text { Triangle } \\
L \in[10] \\
R \in[10]\end{array}$ & & $J_{L Q R}^{A, 1}$ & (A30) \\
\hline
\end{tabular}

TABLE VI. Contribution of all nonvanishing triangle diagrams entering Eq. (29). The meaning of different lines is explained in Fig. 1. 
where the projector $\frac{\bar{\not}+M_{\bar{p}}}{2 M_{\bar{p}}}\left(\frac{\nabla^{+}+M_{p}}{2 M_{p}}\right)$ sets the outgoing (incoming) baryon $\bar{B}(B)$ on shell. The trace is taken in Dirac space. For completeness we also give the expressions $J_{\ldots}^{P}$ and $J_{\ldots}^{I}$, which project onto the form factors $G_{P}$ and $G_{I}$ in Eqs. (A1) and (A2) in Appendix A. Using the method of dimensional regularization [29], we work in $d$ spacetime dimensions.

In Tables IV-VI we show all considered Feynman diagrams with the link to the amplitude $J_{\ldots}^{\mu}$, defined in Appendix A. The resulting projection $J_{\ldots}^{A}$ enters the formula for the axial-vector form factor (29).

We now turn to the flavor structure and take a closer look at the CGCs $C, T, X$, and $G$. The 3-point CGCs $G_{Q R}^{(B)}$ enter the calculation of the baryon masses and are given in Ref. [23], but for completeness we give them in Tables XII-XV. The counterterm CGCs, which originate from the Lagrangian $\mathcal{L}_{3}(17), T_{i}^{(\bar{B} B)}$ and $X_{i}^{(\bar{B} B)}$, are used to absorb the divergences of the one-loop contributions. Explicit symmetry breaking gives rise to the coefficients $X_{i}^{(\bar{B} B)}$, which are proportional to the quark masses $m$ and $m_{s}$. They are shown in Table XVI. The coefficients $T_{i}^{(\bar{B} B)} \propto t=q^{2}$ are important to describe the momentum transfer dependence of the axial-vector current. Their explicit contributions are shown in Table XVII.

The CGCs $C_{\ldots, i}^{(B B)}$ can further be decomposed:

$$
\begin{aligned}
C_{i}^{(\bar{B} B)} & =A_{\bar{B} B}^{(i)}, \\
C_{Q, i}^{(\bar{B} B)} & =A_{Q, i}^{(\bar{B} B)}, \\
C_{L Q, i}^{(\bar{B} B), n} & =A_{Q L,(\bar{B})}^{(i B), n} \tilde{C}_{i}^{\bar{B} B}, \\
C_{Q R, i}^{(\bar{B} B), n} & =A_{Q R,(B)}^{(i \bar{B}), n} \tilde{C}_{\bar{i}}^{B \bar{B}}, \\
C_{L Q R, i}^{(\bar{B} B), 1} & =A_{L R}^{(i)} \tilde{C}_{\bar{B} B, L R}^{i Q},
\end{aligned}
$$

where we defined the 3-, 4-, and 5-point CGCs $A_{\cdots}^{(\cdots)}$ and the recoupling constants $\tilde{C}_{\ldots . . .}$. The 3 -point coefficients $A$ differ from the $G$ coefficients, because they couple an axial-vector current instead of a Goldstone boson to the two baryons. Nevertheless, they only differ in their particle-specific normalization. All needed coefficients $A_{\bar{B} B}^{(i)}$ are given in Tables XVIII-XXI. We note that there are some correlations among such coefficients. For instance, we find

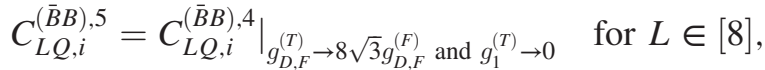

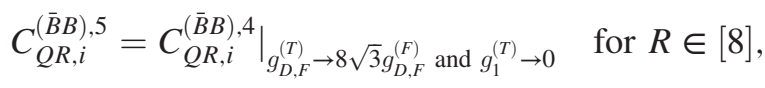

$C_{L Q, i}^{(\bar{B} B), 3}=\left.C_{L Q, i}^{(\bar{B} B), 2}\right|_{f_{i}^{(A)} \rightarrow 0 \text { but } f_{2}^{(A)} \rightarrow 16 f_{F}^{(F)}} \quad$ for $L \in[10]$,

$C_{Q R, i}^{(\bar{B} B), 3}=\left.C_{Q R, i}^{(\bar{B} B), 2}\right|_{f_{i}^{(A)} \rightarrow 0 \text { but } f_{2}^{(A)} \rightarrow 16 f_{F}^{(F)}} \quad$ for $R \in[10]$,

$C_{L Q R, i}^{(\bar{B} B), 2}=\left.C_{L Q R, i}^{(\bar{B} B), 1}\right|_{C \rightarrow-2 f_{E}^{(F)}}$ and $F, D, H \rightarrow 0 \mid$.

The 5-point CGCs $A_{Q, i}^{(\bar{B} B)}$ can directly be linked to the 3-point coefficients $A_{\bar{B} B}^{(i)}$ (see Table XXII). The 4-point vertices contributing to the bubble diagrams require some more attention. First of all, several parts of the Lagrangians $\mathcal{L}_{\text {kin }}$ (5) and $\mathcal{L}^{(2)}$ (9) contribute. This is labeled by the parameter $n$, and the exact mapping can be found in Table V. The 4-point CGCs $A_{Q L,(\bar{B})}^{(i B), n}$ depend, in addition to the connected particles $L, Q, B$, and the current $i$, on the isospin of the outgoing baryon $\bar{B}$. All CGCs $A_{Q L,(\bar{B})}^{(i B), n}$ can be found in the Appendix in Tables XXIII-XXVII and Eqs. (D1) and (D2). The CGCs $A_{Q R,(B)}^{(i \bar{B}), n}$ are linked to $A_{Q L,(\bar{B})}^{(i B), n}$ via the relations given in Eq. (D3).

It turns out that the CGCs $G$ and $A$ are not enough to describe the full flavor structure of all bubble and triangle diagrams. We need additional "recoupling constants" $\tilde{C}$, which compensate for the use of isospin multiplets in the strict isospin limit. Of course, the goal is to be consistent with results in particle basis. We have explicitly checked that this is achieved by the inclusion of the recoupling constants $\tilde{C}$. The recoupling constants of the bubble diagrams $\tilde{C}_{i}^{\bar{B} B}$ depend on external particles only and are given in Table XXVIII. The situation turns slightly more sophisticated for triangle diagrams. The recoupling constants $\tilde{C}_{\bar{B} B, L R}^{i Q}$ depend on all involved particles and are shown in Eqs. (E1), (E2) and Tables XXIX and XXX.

\section{B. Unrenormalized results}

We reduce the expressions for the amplitudes $J_{\ldots}^{A}$ from Eq. (29) by using a Passarino-Veltman reduction scheme [5]. Rewriting our amplitudes in terms of PassarinoVeltman basis integrals and reduced integrals (34) after canceling the occurring kinematic singularities [5], we arrive at the intermediate result in Eq. (33) for amplitudes $J_{\ldots}^{A}$. We apply the notation of Ref. [5] and find 


$$
\begin{aligned}
J^{A}= & 1 \\
J_{Q}^{A}= & K_{Q}^{(Q)} I_{Q}, \\
J_{L Q}^{A, n}= & K_{L Q}^{(Q), n} I_{Q}+K_{L Q}^{(L), n} I_{L}+K_{L Q}^{(L Q), n} I_{L Q}\left(M_{\bar{p}}\right), \\
J_{Q R}^{A, n}= & K_{Q R}^{(Q), n} I_{Q}+K_{Q R}^{(R), n} I_{R}+K_{Q R}^{(Q R), n} I_{Q R}\left(M_{p}\right), \\
J_{L Q R}^{A, n}= & K_{L Q R}^{(Q), n} I_{Q}+K_{L Q R}^{(L), n} I_{L}+K_{L Q R}^{(R), n} I_{R}+K_{L Q R}^{(L Q), n} I_{L Q}\left(M_{\bar{p}}\right)+K_{L Q R}^{(Q R), n} I_{Q R}\left(M_{p}\right)+K_{L Q R}^{(L R), n} I_{L R}(t)+K_{L Q R}^{(L R), n} \frac{\Delta I_{L R}(t)}{t} \\
& +K_{L Q R}^{\prime \prime(L R), n} \frac{\Delta \Delta I_{L R}(t)}{t^{2}}+K_{L Q R}^{(L Q R), n} I_{L Q R}(t)+K_{L Q R}^{(L Q R), n} \frac{\Delta I_{L Q R}(t)}{t-\left(M_{\bar{p}}-M_{p}\right)^{2}},
\end{aligned}
$$

where the amplitude $J_{a b c}^{A}$, which originates from a diagram with internal particles $a, b, c$, is expressed by a combination of prefactor and integral $K_{a b c}^{(A B C)} I_{A B C}$. Note that the Passarino-Veltman reduction scheme leads to the appearance of integrals with internal particles $A B C$, which differ from the internal particles of the original diagram $a b c$. The prefactors $K_{a b c}^{(A B C)}$ are too long to be shown here in full detail. The prefactors $K^{\prime}$ and $K^{\prime \prime}$ are linked to the reduced integrals $\Delta I \ldots$ and $\Delta \Delta I \ldots$,

$$
\begin{aligned}
I_{R} & =\int \frac{d^{d} l}{(2 \pi)^{d}} \frac{i \mu^{4-d}}{l^{2}-M_{R}^{2}}, \\
I_{Q} & =\int \frac{d^{d} l}{(2 \pi)^{d}} \frac{i \mu^{4-d}}{l^{2}-m_{Q}^{2}}, \\
I_{L} & =\int \frac{d^{d} l}{(2 \pi)^{d}} \frac{i \mu^{4-d}}{l^{2}-M_{L}^{2}}, \\
I_{L Q}\left(\bar{p}^{2}\right) & =\int \frac{d^{d} l}{(2 \pi)^{d}} \frac{-i \mu^{4-d}}{\left((l-\bar{p})^{2}-M_{L}^{2}\right)\left(l^{2}-m_{Q}^{2}\right)}, \\
I_{L R}(t) & =\int \frac{d^{d} l}{(2 \pi)^{d}} \frac{-i \mu^{4-d}}{\left((l-(\bar{p}-p))^{2}-M_{L}^{2}\right)\left(l^{2}-M_{R}^{2}\right)}, \\
I_{Q R}\left(p^{2}\right) & =\int \frac{d^{d} l}{(2 \pi)^{d}} \frac{-i \mu^{4-d}}{\left(l^{2}-m_{Q}^{2}\right)\left((l-p)^{2}-M_{R}^{2}\right)}, \\
I_{L Q R}\left(\bar{p}^{2}, p^{2}, t\right) & =\int \frac{d^{d} l}{(2 \pi)^{d}} \frac{i \mu^{4-d}}{\left((l-\bar{p})^{2}-M_{L}^{2}\right)\left(l^{2}-m_{Q}^{2}\right)\left((l-p)^{2}-M_{R}^{2}\right)}, \\
\Delta I_{L R}(t) & =I_{L R}(t)-I_{L R}(t=0), \\
\Delta \Delta I_{L R}(t) & =I_{L R}(t)-I_{L R}(t=0)-\left.t \frac{\partial}{\partial t} I_{L R}(t)\right|_{t=0}, \\
\Delta I_{L Q R}(t) & =I_{L Q R}(t)-I_{L Q R}\left(t=\left(M_{\bar{p}}-M_{p}\right)^{2}\right),
\end{aligned}
$$

with

$$
\begin{aligned}
I_{L R}(t=0) & =\frac{I_{R}-I_{L}}{M_{L}^{2}-M_{R}^{2}}, \\
\left.\frac{\partial}{\partial t} I_{L R}(t)\right|_{t=0} & =\frac{\left((d-4) M_{L}^{2}-d M_{R}^{2}\right)}{d\left(M_{L}^{2}-M_{R}^{2}\right)^{3}} I_{L}+\frac{\left(d M_{L}^{2}-(d-4) M_{R}^{2}\right)}{d\left(M_{L}^{2}-M_{R}^{2}\right)^{3}} I_{R}, \\
I_{L Q R}\left(t=\left(M_{\bar{p}}-M_{p}\right)^{2}\right) & =\frac{M_{\bar{p}}\left(I_{L Q}\left(M_{\bar{p}}\right)-I_{L R}\left(\left(M_{\bar{p}}-M_{p}\right)^{2}\right)\right)-M_{p}\left(I_{Q R}\left(M_{p}\right)-I_{L R}\left(\left(M_{\bar{p}}-M_{p}\right)^{2}\right)\right)}{M_{p}\left(M_{\bar{p}}^{2}+m_{Q}^{2}-M_{L}^{2}\right)-M_{\bar{p}}\left(M_{p}^{2}+m_{Q}^{2}-M_{R}^{2}\right)} .
\end{aligned}
$$




\section{RENORMALIZATION AND POWER COUNTING}

Applying the general strategy of Refs. [5,32] we will use the following basic guidelines for renormalization and power counting:

(i) the use of on-shell masses in the loop contributions,

(ii) introduction of a consistent power-counting scheme with special attention to the decuplet-octet mass difference,

(iii) no expansion of the integrals $I$, but the use of appropriate subtractions in order to preserve power counting,

(iv) a consistent expansion of the prefactors $K$ in appropriate variables.

In Eq. (33) the integrals $I_{\ldots}$ and their prefactors $K_{(\cdots)}^{(\cdots)}$ depend on both external masses, $M_{\bar{p}}$ and $M_{p}$, and internal ones $M_{L}, M_{R}$, and $m_{Q}$. There are different approaches on how to treat these internal masses. Ledwig et al. [33] use physical meson masses and present two options for the baryon masses. First, the octet and decuplet masses are set to their values in the chiral limit ( $M$ and $M+\Delta$ ), whereas their second option is the average over the physical octet and the decuplet masses. Whether any of these simplifications is appropriate to describe QCD lattice data at unphysically large meson masses to high precision appears questionable.

Here we will use on-shell masses in the loop contributions, following the path demonstrated for baryon masses [32]. It is a self-consistent approach, which is based on the eight coupled equations for the baryon octet and decuplet masses:

$$
\begin{aligned}
M_{N}= & M+\tilde{\Sigma}_{N}\left(M_{N}, M_{\Lambda}, M_{\Sigma}, M_{\Xi}, M_{\Delta_{\mu}}, M_{\Sigma_{\mu}}, M_{\Xi_{\mu}},\right. \\
& \left.M_{\Omega_{\mu}}, m_{\pi}, m_{K}, m_{\bar{K}}, m_{\eta}\right), \\
M_{\Sigma}= & M+\tilde{\Sigma}_{\Sigma}\left(M_{N}, M_{\Lambda}, M_{\Sigma}, M_{\Xi}, M_{\Delta_{\mu}}, M_{\Sigma_{\mu}}, M_{\Xi_{\mu}},\right. \\
& \left.M_{\Omega_{\mu}}, m_{\pi}, m_{K}, m_{\bar{K}}, m_{\eta}\right), \\
M_{\Lambda}= & \cdots \\
& \vdots \\
M_{\Omega_{\mu}}= & M+\Delta+\tilde{\Sigma}_{\Omega_{\mu}}\left(M_{N}, M_{\Lambda}, M_{\Sigma}, M_{\Xi}, M_{\Delta_{\mu}}, M_{\Sigma_{\mu}},\right. \\
& \left.M_{\Xi_{\mu}}, M_{\Omega_{\mu}}, m_{\pi}, m_{K}, m_{\bar{K}}, m_{\eta}\right),
\end{aligned}
$$

where $\tilde{\Sigma}_{A}$ is the self-energy to the mass of an octet or decuplet particle $A$, which depends on internal loop masses. In conventional $\chi \mathrm{PT}$, e.g. Refs. [34,35], the masses on the right-hand side of Eq. (36) are expanded in order to decouple the equations. In contrast to this, Lutz et al. [32] keep the full structure on the right-hand side of Eq. (36) and solve the set of coupled equations. This self-consistent approach leads to a very good description of octet and decuplet masses up to large meson masses of $600 \mathrm{MeV}$ [11]. We expect that the use of these accurate onshell masses allows an improved description of the loop contributions to the axial-vector form factors as well.
The use of on-shell masses requires a clear strategy regarding power counting. In principle, the triangle integral $I_{L Q R}\left(M_{\bar{p}}, M_{p}\right)$ can involve four different baryon masses, $M_{\bar{p}}, M_{p}, M_{L}$, and $M_{R}$. Although $M_{\bar{p}}$ and $M_{p}$ are restricted to octet masses, the fact that $M_{L}$ and $M_{R}$ can be octet and decuplet masses complicates the question how to deal with baryon mass differences in our framework. In contrast, previous works expanded the masses in the loop contributions and were therefore mainly confronted with the treatment of the nonzero mass difference between the decuplet and the octet baryons in the chiral limit $\Delta$. The small-scale expansion [31] uses an expansion in $\Delta$. Using the explicit example of the bubble integral $I_{Q R}\left(M_{p}\right)$, the poor convergence of this expansion has been demonstrated in Ref. [32]. Therefore, we do not expand in $\Delta$. Our main strategy is to subtract the terms that violate power counting in any of the two regions $m_{Q}<\Delta$ and $m_{Q} \simeq \Delta$. To cope with the four different baryon masses, we rewrite them in terms of mass differences $\delta_{B}, \delta_{L}, \delta_{R}$ :

$$
\begin{aligned}
\delta_{B} & =M_{\bar{p}}-M_{p}, \quad \delta_{L}=M_{L}-M_{\bar{p}}\left(1+\gamma_{L}\right), \\
M_{B} & =\frac{M_{\bar{p}}+M_{p}}{2}, \quad \delta_{R}=M_{R}-M_{p}\left(1+\gamma_{R}\right), \\
\gamma_{L} & = \begin{cases}0 & \text { if } M_{L} \in[8], \\
\Delta / M & \text { if } M_{L} \in[10],\end{cases} \\
\gamma_{R} & = \begin{cases}0 & \text { if } M_{R} \in[8], \\
\Delta / M & \text { if } M_{R} \in[10] .\end{cases}
\end{aligned}
$$

The subtractions $\gamma_{L}$ and $\gamma_{R}$ are chosen in such a way that $\delta_{L}$ and $\delta_{R}$ vanish in the chiral limit for all cases $L / R \in\{[8],[10]\}$. As we restrict external particles to the baryon octet, $\delta_{B}$ vanishes in the chiral limit without any subtractions needed.

In the following we turn to the explicit renormalization rules for the amplitudes in Eq. (33):

$$
J_{\ldots}^{A} \rightarrow \bar{J}_{\ldots}^{A} \quad \text { with } \quad I_{\ldots} \rightarrow \bar{I}_{\ldots .} \quad \text { and } \quad K_{\ldots}^{(\cdots)} \rightarrow \bar{K}_{\ldots}^{(\cdots)} .
$$

Equation (33) can be rewritten in terms of renormalized integrals $\bar{I}_{\ldots}$ and prefactors $\bar{K}_{\ldots}^{(\cdots)}$ :

$$
\begin{aligned}
\bar{J}^{A}= & 1, \\
\bar{J}_{Q}^{A}= & \bar{K}_{Q}^{(Q)} \bar{I}_{Q}, \\
\bar{J}_{L Q}^{A, n}= & \bar{K}_{L Q, n}^{(Q),} \bar{I}_{Q}+\bar{K}_{L Q}^{(L Q), n} \bar{I}_{L Q}\left(M_{\bar{p}}\right), \\
\bar{J}_{Q R}^{A, n}= & \bar{K}_{Q R}^{(Q), n} \bar{I}_{Q}+\bar{K}_{Q R}^{(Q R), n} \bar{I}_{Q R}\left(M_{p}\right), \\
\bar{J}_{L Q R}^{A, n}= & \bar{K}_{L Q R}^{(Q), n} \bar{I}_{Q}+\bar{K}_{L Q R}^{(L Q), n} \bar{I}_{L Q}\left(M_{\bar{p}}\right)+\bar{K}_{L Q R}^{(Q R), n} \bar{I}_{Q R}\left(M_{p}\right) \\
& +\bar{K}_{L Q R}^{(L Q R), n} \bar{I}_{L Q R}(t)+\bar{K}_{L Q R}^{(L Q R), n} \frac{\Delta \bar{I}_{L Q R}(t)}{t-\left(M_{\bar{p}}-M_{p}\right)^{2}} .
\end{aligned}
$$


Note that the integrals $I_{L}, I_{R}, I_{L R}, \Delta I_{L R}$, and $\Delta \Delta I_{L R}$ do not appear in Eq. (39) anymore [see Eq. (42) and the discussion above].

We apply as power-counting rules

$$
\begin{aligned}
& m_{Q}^{2} \sim Q^{2}, \quad t \sim Q^{2}, \quad M_{B} \sim Q^{0}, \\
& \delta_{B} \sim Q^{2}, \quad \delta_{L} \sim Q^{2}, \quad \delta_{R} \sim Q^{2} .
\end{aligned}
$$

The prefactors $\bar{K}_{(\cdots)}^{(\cdots)}$ are obtained from $K_{(\cdots)}^{(\cdots)}$ by subtracting power-counting violating terms $\delta K_{(\cdots)}^{(\cdots)}$ :

$$
\bar{K}^{(\cdots)}=K^{(\cdots)}-\delta K^{(\cdots)} .
$$

All factors $K_{(\cdots)}^{(\cdots)}, \bar{K}_{(\cdots)}^{(\cdots)}$, and $\delta K_{(\cdots)}^{(\cdots)}$ are rewritten in terms of the new variables, defined in Eq. (37). The unrenormalized, full factors $K_{(\cdots)}^{(\ldots)}$ are expanded following the power-counting rules in Eq. (40). The renormalized coefficients $\bar{K}_{(\cdots)}^{(\cdots)}$ are explicitly given in Appendix (F1)-(F4) and involve contributions proportional to factors $\alpha_{i j}$, which are functions of the ratio $\Delta / M$ only and normalized to $\alpha_{i j} \rightarrow 1$ for $\Delta \rightarrow 0$. An expansion in $\Delta / M$, such as performed in Ref. [31], would lead to $\alpha_{i j} \rightarrow 1$ at leading order. We insist on keeping the full factors $\alpha_{i j}$ and demonstrate in Ref. [5] that $\alpha_{i j}=1$ is a very poor approximation for some $i j$. Equation (F5) in Appendix F lists all $\alpha_{i j}$. Power-counting violating terms $\delta K^{(\cdots)}$ are subtracted and denoted by an index $0: \alpha_{0 j} \rightarrow 0$ and $\alpha_{j 0} \rightarrow 0$. All power-counting violating, and therefore subtracted terms, $\delta K^{(\cdots)}$ are given in Eqs. (44), (45), and (49).

The renormalized integrals $\bar{I}_{\text {... }}$, which we want to keep unchanged, if power counting allows, are not expressed in the new variables. We will develop their explicit structure in the following.

Integrals that do not involve any meson mass consist of large scales only, because we do not consider $\Delta$ as a small scale. Therefore, it was argued in Ref. [23] that these contributions should be fully subtracted, so they do not appear in Eq. (39) anymore:

$$
\bar{I}_{L}=\bar{I}_{R}=\bar{I}_{L R}=\Delta \bar{I}_{L R}=\Delta \Delta \bar{I}_{L R}=0 .
$$

The reduced integrals $\Delta I_{L R}$ and $\Delta \Delta I_{L R}$ were defined in Eqs. (34).

The renormalized meson tadpole $\bar{I}_{Q}$ is of order $Q^{2}$,

$$
\bar{I}_{Q}=\frac{m_{Q}^{2}}{16 \pi^{2}} \log \left[\frac{m_{Q}^{2}}{\mu^{2}}\right] .
$$

It is the only remaining source of the renormalization scale $\mu$. We expand its prefactor $K_{.}^{(Q)}$, but consider only order- $Q^{0}$ terms, since higher-order terms are expected to be renormalized by counterterms of order $Q^{4}$ and higher. As diagrams with an internal decuplet are not expected to contribute to this order, their contributions $\delta K_{(Q)}^{(Q)}$, proportional to $\alpha_{0 j}$ with $j \in\{1,2,3,4\}$, are subtracted via

$$
\begin{aligned}
\delta K_{L \in[10] Q R \in[8]}^{(Q), n=1} & =\delta K_{L \in[8] Q R \in[10]}^{(Q), n=1}=\frac{1}{3} \alpha_{01}, \\
\delta K_{L \in[10] Q R \in[10]}^{(Q), n=1} & =-\frac{4}{9} \alpha_{02}, \\
\delta K_{L \in[10] Q}^{(Q), n=1} & =\delta K_{Q R \in[10]}^{(Q), n=1}=\frac{10}{9} \frac{\Delta}{M} \alpha_{03} M_{B}, \\
\delta K_{L \in[10] Q}^{(Q), n=2} & =\delta K_{Q R \in[10]}^{(Q), n=2}=-\frac{2}{9} \frac{\Delta}{M} \alpha_{04} M_{B} .
\end{aligned}
$$

The discussion becomes more complex for the bubble integrals evaluated at external baryon masses $\bar{I}_{L Q}\left(M_{\bar{p}}\right)$ and $\bar{I}_{Q R}\left(M_{p}\right)$. If $L, R \in[8]$, no subtractions are necessary, but in case $L, R \in[10]$ power-counting violating terms arise. Therefore we subtract all $Q^{0}$-contributions $\delta K^{(L Q)}$ and $\delta K^{(Q R)}$ in Eq. (45) by the rule $\alpha_{j 0} \rightarrow 0$ for $j=1-5$. For instance,

$$
\begin{gathered}
\delta K_{L \in[8] Q R \in[10]}^{(L Q), n=1}=\delta K_{L \in[10] Q R \in[8]}^{(Q R), n=1}=-\frac{5}{12} \frac{\Delta}{M} \alpha_{10} M_{B}^{2}, \\
\delta K_{L \in[10] Q R \in[8]}^{(L Q), n=1}=\delta K_{L \in[8] Q R \in[10]}^{(Q R), n=1}=-\frac{5}{12} \frac{\Delta}{M} \alpha_{20} M_{B}^{2}, \\
\delta K_{L \in[10] Q R \in[10]}^{(L Q), n=1}=\delta K_{L \in[10] Q R \in[10]}^{(Q R), n=1}=\frac{7}{9} \frac{\Delta}{M} \alpha_{30} M_{B}^{2}, \\
\delta K_{L \in[10] Q}^{(L Q), n=1}=\delta K_{Q R \in[10]}^{(Q R), n=1}=-\frac{20}{9} \frac{\Delta^{2}}{M^{2}} \alpha_{40} M_{B}^{3}, \\
\delta K_{L \in[10] Q}^{(L Q), n=2}=\delta K_{Q R \in[10]}^{(Q R), n=2}=\frac{4}{9} \frac{\Delta^{2}}{M^{2}} \alpha_{50} M_{B}^{3} .
\end{gathered}
$$

These subtractions are not enough to eliminate all power-counting violating contributions. Additional subtractions are needed for the integrals $\bar{I}_{L Q}$ and $\bar{I}_{Q R}$. We therefore introduce the subtractions $\gamma_{\bar{B}}^{L}$ and $\gamma_{B}^{R}$, which assure the correct power counting $\bar{I}_{L \in[8] Q} / \bar{I}_{Q R \in[8]} \sim Q^{1}$ and $\bar{I}_{L \in[10] Q} / \bar{I}_{Q R \in[10]} \sim Q^{2}$ in the chiral domain [32]:

$$
\begin{aligned}
& \bar{I}_{L Q}\left(M_{\bar{p}}\right)=I_{L Q}\left(M_{\bar{p}}\right)+\frac{I_{L}}{M_{L}^{2}}-\frac{1-\gamma_{\bar{B}}^{L}}{16 \pi^{2}}, \\
& \bar{I}_{Q R}\left(M_{p}\right)=I_{Q R}\left(M_{p}\right)+\frac{I_{R}}{M_{R}^{2}}-\frac{1-\gamma_{B}^{R}}{16 \pi^{2}},
\end{aligned}
$$

where $\gamma_{\bar{B}}^{L}$ and $\gamma_{B}^{R}$ depend on baryon masses in the chiral limit only:

$$
\gamma_{B}^{R}= \begin{cases}0 & \text { if } R \in[8], \\ -\frac{2 M \Delta+\Delta^{2}}{M^{2}} \log \left[\frac{2 M \Delta+\Delta^{2}}{(M+\Delta)^{2}}\right] & \text { if } R \in[10] .\end{cases}
$$

The explicit form of $\bar{I}_{Q R}\left(p^{2}=M_{p}^{2}\right)$ has been determined in Ref. [32]: 


$$
\begin{aligned}
\bar{I}_{Q R}\left(M_{p}\right)= & \frac{1}{16 \pi^{2}}\left[\gamma_{B}^{R}-\left(\frac{1}{2}+\frac{m_{Q}^{2}-M_{R}^{2}}{2 M_{p}^{2}}\right) \log \left[\frac{m_{Q}^{2}}{M_{R}^{2}}\right]\right. \\
& +\frac{p_{Q R}}{M_{p}}\left(\log \left[1-\frac{M_{p}^{2}-2 p_{Q R} M_{p}}{m_{Q}^{2}+M_{R}^{2}}\right]\right. \\
& \left.\left.-\log \left[1-\frac{M_{p}^{2}+2 p_{Q R} M_{p}}{m_{Q}^{2}+M_{R}^{2}}\right]\right)\right], \\
p_{Q R}^{2}= & \frac{M_{p}^{2}}{4}-\frac{M_{R}^{2}+m_{Q}^{2}}{2}+\frac{\left(M_{R}^{2}-m_{Q}^{2}\right)^{2}}{4 M_{p}^{2}} .
\end{aligned}
$$

It is left to discuss the contributions of the triangle integrals $I_{L Q R}(t)$ and $\Delta I_{L Q R}(t)$. Analogous to the bubbles, power-counting violating terms only appear in the presence of internal decuplet particles. This is true for the cases $L \in[10], R \in[8]$ as well as for $L \in[8], R \in[10]$, and $L, R \in[10]$. Therefore, the order- $Q^{0}$ contributions $\delta K^{(L Q R)}$ in Eq. (49), associated with $\alpha_{j 0} \rightarrow 0$ with $j \in\{6,7,8\}$, are subtracted. For instance,

$$
\begin{gathered}
\delta K_{L \in[10] Q R \in[8]}^{(L Q R), 1}=\delta K_{L \in[8] Q R \in[10]}^{(L Q R), 1}=\frac{5}{6} \frac{\Delta^{2}}{M^{2}} \alpha_{60} M_{B}^{4}, \\
\delta K_{L \in[10] Q R \in[8]}^{\prime(L Q R), 1}=\delta K_{L \in[8] Q R \in[10]}^{\prime(L Q R), 1}=\frac{2}{3} \frac{\Delta^{2}}{M^{2}} \alpha_{70} M_{B}^{6}, \\
\delta K_{L \in[10] Q R \in[10]}^{(L Q R), 1}=-\frac{4}{3} \frac{\Delta^{2}}{M^{2}} \alpha_{80} M_{B}^{4} .
\end{gathered}
$$

As the triangle integrals $I_{L Q R}(t)$ and $\Delta I_{L Q R}(t)$, defined in Eq. (34), are finite for $d=4$, renormalization is not needed. Only power-counting violating contributions of the integrals need to be eliminated by introducing the subtractions $\gamma_{L Q R}$ and $\gamma_{L Q R}^{\prime}[5]$ :

$$
\begin{gathered}
\bar{I}_{L Q R}(t)=I_{L Q R}(t)-\gamma_{L Q R}, \\
\gamma_{L \in[10] Q R \in[8]}=\gamma_{L \in[8] Q R \in[10]} \\
=-\frac{1}{16 \pi^{2} M^{2}} \log \left[\frac{\left.2 M \Delta+\Delta^{2}\right]}{(M+\Delta)^{2}}\right]+\frac{1}{16 \pi^{2}\left(2 M \Delta+\Delta^{2}\right)} \log \left[\frac{(M+\Delta)^{2}}{M^{2}}\right], \\
\gamma_{L \in[8] Q R \in[8]}=0, \quad \gamma_{L \in[10] Q R \in[10]}=-\frac{1}{16 \pi^{2} M^{2}} \log \left[\frac{2 M \Delta+\Delta^{2}}{(M+\Delta)^{2}}\right], \\
\bar{I}_{L Q R}(t)=\bar{I}_{L Q R}(t)-\bar{I}_{L Q R}\left(\left(M_{\bar{p}}-M_{p}\right)^{2}\right)-\left(t-\left(M_{\bar{p}}-M_{p}\right)^{2}\right) \gamma_{L Q R}^{\prime}, \\
\gamma_{L \in[10] Q R \in[8]}^{\prime}=\gamma_{L \in[8] Q R \in[10]}^{\prime}=-\frac{1}{96 \pi^{2} M^{4} \Delta^{3}(2 M+\Delta)^{3}}\left(\Delta^{3}(2 M+\Delta)^{3} \log \left[\frac{\Delta}{M}\right]\right. \\
-2(M+\Delta)^{2} \log \left[\frac{M+\Delta}{M}\right]\left(4 M^{4}-2 M^{3} \Delta+3 M^{2} \Delta^{2}+4 M \Delta^{3}+\Delta^{4}\right) \\
\left.+\Delta(2 M+\Delta)\left(M^{2}\left(4 M^{2}+2 M \Delta+\Delta^{2}\right)+\Delta^{2}(2 M+\Delta)^{2} \log \left[\frac{2 M+\Delta}{M}\right]\right)\right), \\
\gamma_{L \in[8] Q R \in[8]}^{\prime}= \\
\gamma_{L \in[10] Q R \in[10]}^{\prime}=0 .
\end{gathered}
$$

The subtractions $\gamma_{L Q R}$ and $\gamma_{L Q R}^{\prime}$ are analogous to the subtraction term $\gamma_{R}^{B}$ introduced in the bubble function. With Eqs. (50) and (51) we obtain $\bar{I}_{L \in[8] Q R \in[8]}(t) \sim Q^{0}, \quad \bar{I}_{L \in[8] Q R \in[10]}(t) \sim Q^{1}$, and $\bar{I}_{L \in[10] Q R \in[10]}(t) \sim Q^{2}$ and $\quad \Delta \bar{I}_{L \in[8] Q R \in[8]}(t) /\left(t-\left(M_{\bar{p}}-M_{p}\right)^{2}\right) \sim Q^{0}, \quad \Delta \bar{I}_{L \in[8] Q R \in[10]}(t) /\left(t-\left(M_{\bar{p}}-M_{p}\right)^{2}\right) \sim Q^{1}, \quad$ and $\Delta \bar{I}_{L \in[10] Q R \in[10]}(t) /(t-$ $\left.\left(M_{\bar{p}}-M_{p}\right)^{2}\right) \sim Q^{2}$ in the chiral domain. The subtractions are crucial, since otherwise the explicit evaluation of a class of two-loop diagrams would be needed [5,36]. An explicit expression for the triangle integral at $t=\left(M_{\bar{p}}-M_{p}\right)^{2}$ was given in Eq. (35). For general values of the momentum transfer $t$ we use the Feynman parametrization

$$
\begin{aligned}
\bar{I}_{L Q R}(t) & =-\int_{0}^{1} \int_{0}^{1-u} d v d u \frac{1}{(4 \pi)^{2} \Omega^{2}}-\gamma_{L Q R}, \\
\text { with } \Omega^{2} & =-m_{Q}^{2}+v\left((1-v) M_{p}^{2}-M_{R}^{2}+m_{Q}^{2}\right)+u\left((1-u) M_{\bar{p}}^{2}-M_{L}^{2}+m_{Q}^{2}\right)+u v\left(t-M_{\bar{p}}^{2}-M_{p}^{2}\right),
\end{aligned}
$$

from which also the reduced triangle integral $\Delta \bar{I}_{L Q R}(t)$ follows with Eq. (51). 
Our renormalized and power-counting respecting result for the axial-vector form factors $G_{A, i}^{\bar{B} B}(t)$ is summarized in Eq. (53). It uses the renormalized amplitudes from Eq. (39) plugged into Eq. (29) and rearranged by renormalized integrals instead of diagrams:

$$
\begin{aligned}
(2 f)^{2} G_{A, i}^{\bar{B} B}(t)= & (2 f)^{2}\left(\sqrt{Z_{\bar{B}} Z_{B}} C_{i}^{(\bar{B} B)}+t T_{i}^{(\bar{B} B)}+2 B_{0} X_{i}^{(\bar{B} B)}\right)+\sum_{Q \in[8]} \bar{I}_{Q}\left(C_{Q, i}^{(\bar{B} B)} \bar{K}_{Q}^{(Q)}+\sum_{L \in[8],[10]} G_{Q L}^{(\bar{B})} C_{L Q, i}^{(\bar{B} B), n} \bar{K}_{L Q}^{(Q), n}\right. \\
& \left.+\sum_{R \in[8],[10]} C_{Q R, i}^{(\bar{B} B), n} G_{Q R}^{(B)} \bar{K}_{Q R}^{(Q), n}+\sum_{L, R \in[8],[10]} G_{Q L}^{(\bar{B})} C_{L Q R, i}^{(\bar{B} B), n} G_{Q R}^{(B)} \bar{K}_{L Q R}^{(Q), n}\right) \\
& +\sum_{Q \in[8]} \sum_{L \in[8],[10]} \bar{I}_{L Q}\left(M_{\bar{p}}\right)\left(G_{Q L}^{(\bar{B})} C_{L Q, i}^{(\bar{B} B), n} \bar{K}_{L Q}^{(L Q), n}+\sum_{R \in[8],[10]} G_{Q L}^{(\bar{B})} C_{L Q R, i}^{(\bar{B} B), n} G_{Q R}^{(B)} \bar{K}_{L Q R}^{(L Q), n}\right) \\
& +\sum_{Q \in[8]} \sum_{R \in[8],[10]} \bar{I}_{Q R}\left(M_{p}\right)\left(C_{Q R, i}^{(\bar{B} B), n} G_{Q R}^{(B)} \bar{K}_{Q R}^{(Q R), n}+\sum_{L \in[8],[10]} G_{Q L}^{(\bar{B})} C_{L Q R, i}^{(\bar{B} B), n} G_{Q R}^{(B)} \bar{K}_{L Q R}^{(Q R), n}\right) \\
& +\sum_{Q \in[8]} \sum_{L, R \in[8],[10]} \bar{I}_{L Q R}(t)\left(G_{Q L}^{(\bar{B})} C_{L Q R, i}^{(\bar{B} B), n} G_{Q R}^{(B)} \bar{K}_{L Q R}^{(L Q R), n}\right) \\
& +\sum_{Q \in[8]} \sum_{L, R \in[8],[10]} \frac{\Delta \bar{I}_{L Q R}(t)}{t-\left(M_{\bar{p}}-M_{p}\right)^{2}}\left(G_{Q L}^{(\bar{B})} C_{L Q R, i}^{(\bar{B} B), n} G_{Q R}^{(B)} \bar{K}_{L Q R}^{(L Q R), n}\right),
\end{aligned}
$$

where a summation of repeated indices $n$ is understood.

\section{NUMERICAL ANALYSIS OF CONVERGENCE PROPERTIES}

\section{A. Convergence properties at the physical point}

The 12 independent axial-vector form factors of the baryon octet in Eq. (53) are ready to be compared to experimental and QCD lattice data. In Table II we have shown the six existing experimental data points. Unfortunately, the available QCD lattice data for the hyperons $\Sigma, \quad \Xi$, and $\Lambda$ are very limited. In Refs. [15,25,26], results for the form factors $G_{A, a_{\mu}^{\pi}}^{\Sigma \Sigma}(t=0)$ and $G_{A, a_{\mu}^{\pi}}^{\Xi \Xi}(t=0)$ have been presented. However, these data points are not enough to determine all LECs of our chiral Lagrangian. On the other hand, further investigations of axial-vector form factors were announced in Ref. [15]. Until these data become available, we use the LECs determined from fits of the baryon octet and decuplet masses [11] and test some properties of our results for the axial-vector form factors from Eq. (53) at a more elementary level.

First we scrutinize the convergence properties of the kinematic factors $\bar{K}$ of the one-loop contributions in Eq. (53), which are expanded according to the powercounting scheme presented in Eq. (40). We use isospinaveraged physical on-shell masses in the loop contributions, as listed in the left column in Table VII.

In Fig. 2 we show the one-loop contributions to the axialvector form factors $G_{A, i}^{\bar{B} B}\left(t=\left(M_{\bar{p}}-M_{p}\right)^{2}\right)$, but restrict the used LECs to the lowest order from $\mathcal{L}^{(1)}$ in Eq. (7). We do not take into account the LECs from $\mathcal{L}^{(2)}$ and $\mathcal{L}^{(3)}$ in Eqs. (9) and (10), because not all of them are known. Furthermore, we add the order- $Q^{2}$ impact of the wavefunction renormalization to the tree-level contributions $\left(\sqrt{Z_{\bar{B}} Z_{B}}-1\right) C_{i}^{(\bar{B} B)}$. The used parameters are shown in the right column of Table VII.

We divide the 12 independent processes $(\bar{B} \leftarrow B)$ in Fig. 2 with respect to their axial-vector current. In the two top panels the strangeness-conserving axial-vector currents $a_{\mu}^{\pi}$ and $a_{\mu}^{\eta}$ are shown and the strangeness-changing current $a_{\mu}^{K}$ is presented in the bottom of Fig. 2. Dashed lines

TABLE VII. Physical masses [24] and input parameters for the numerical estimates in flavor-SU(3). The LECs refer to "set 1 " of Ref. [11], whereas the factors $Z_{B}$, with $B \in[8]$, are taken from Ref. [32].

\begin{tabular}{lccc}
\hline \hline Masses (Mev) & PDG [24] & \multicolumn{2}{c}{ Parameters from [11,32] } \\
\hline$m_{\pi}$ & 137 & $F$ & 0.48 \\
$m_{K}$ & 494 & $D$ & 0.75 \\
$m_{\eta}$ & 548 & $C$ & 1.50 \\
$M_{N}$ & & $H$ & 2.06 \\
$M_{\Lambda}$ & 939 & $f(\mathrm{MeV})$ & 92.4 \\
$M_{\Sigma}$ & 1116 & $M(\mathrm{MeV})$ & 866.2 \\
$M_{\Xi}$ & 1193 & $\Delta(\mathrm{MeV})$ & 382.7 \\
$M_{\Delta_{\mu}}$ & 1318 & $\mu(\mathrm{MeV})$ & 770 \\
$M_{\Sigma_{\mu}}$ & 1232 & $Z_{N}$ & 1.118 \\
$M_{\Xi_{\mu}}$ & 1385 & $Z_{\Lambda}$ & 2.064 \\
$M_{\Omega_{\mu}}$ & 1533 & $Z_{\Sigma}$ & 2.507 \\
\hline \hline
\end{tabular}



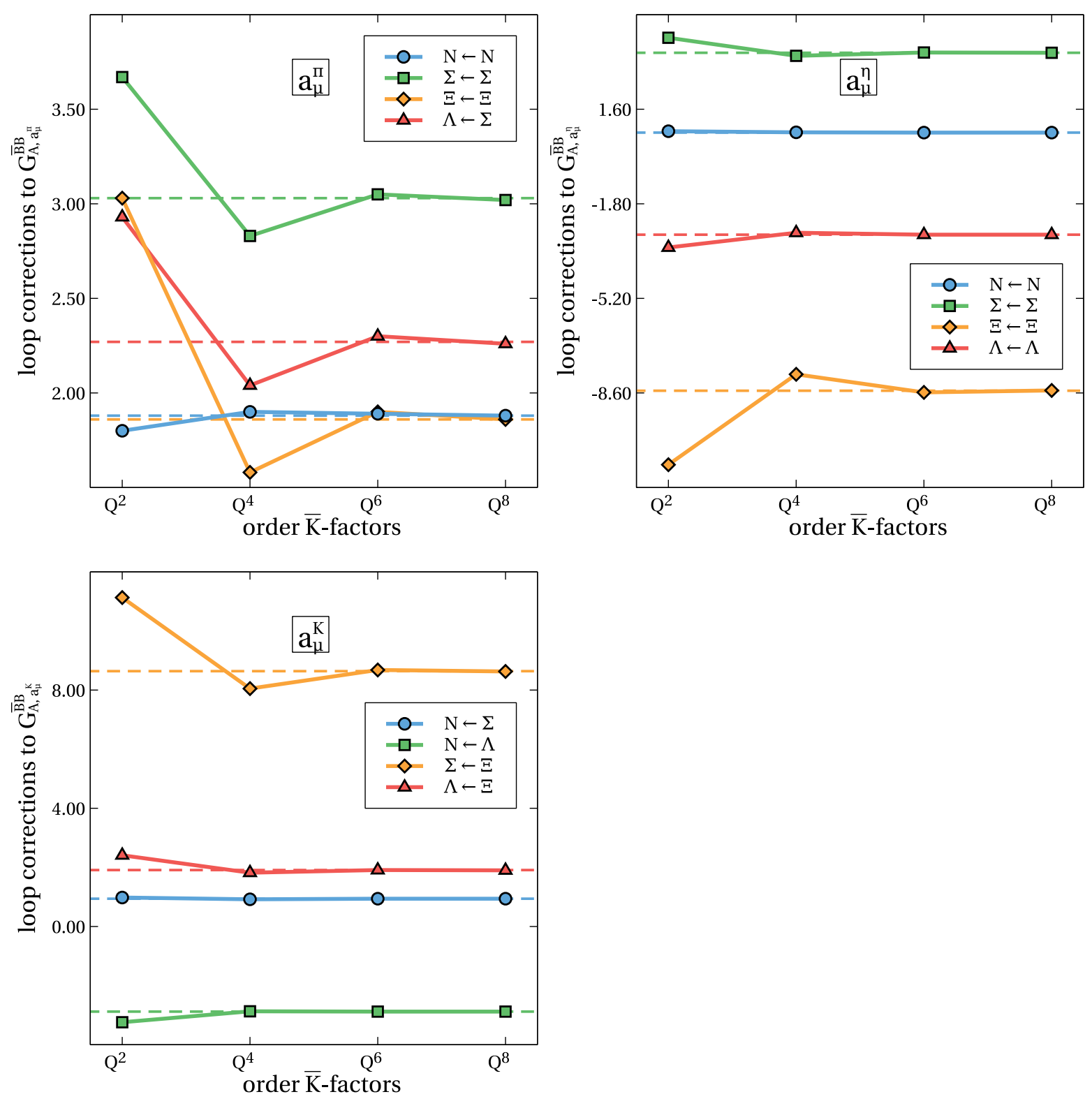

FIG. 2. Convergence properties of the kinematic factors $\bar{K}$. We show their contributions to $G_{A, i}^{\bar{B} B}\left(t=\left(M_{\bar{p}}-M_{p}\right)^{2}\right)$ for the chiral orders $Q^{2}, Q^{4}, Q^{6}$, and $Q^{8}$ as points and their unexpanded value as a dashed line.

represent the full, nonexpanded expression for the kinematic factors $\bar{K}$, whereas the points give the values for the expanded factors up to chiral order $Q^{2}, Q^{4}, Q^{6}$, and $Q^{8}$. These orders refer to the kinematic factors $\bar{K}$ only, the order of the referring integrals $\bar{I}$ is not taken into account here. This restricts the discussion to the convergence properties of the kinematic factors $\bar{K}$. A discussion of the convergence properties of the loop contributions in general is performed below. The higher-order terms $Q^{6}$ and $Q^{8}$ reproduce the full contributions $\bar{K}$ for all processes to high accuracy, and the order- $Q^{4}$ terms show reasonable results. However, only taking into account the order- $Q^{2}$ terms does not appear desirable, except for the processes with at least one external nucleon. In general, we find large loop contributions compared to the tree-level values of Table II.

In order to scrutinize the full convergence properties of our chiral expansion, we also take into account the chiral power of the integrals $\bar{I}_{Q} \sim Q^{2}, \bar{I}_{L Q} / \bar{I}_{Q R} \sim Q^{1}$, and $\bar{I}_{L Q R} \sim Q^{0}$. We use the momentum transfer $t=$ $\left(M_{\bar{p}}-M_{p}\right)^{2}$, implying that $\Delta \bar{I}_{L Q R}\left(\left(M_{\bar{p}}-M_{p}\right)^{2}\right)=0$. The tadpole integral $\bar{I}_{Q}$ still involves the renormalization scale $\mu$ (43). Therefore all contributions $\bar{K}^{(Q)} \bar{I}_{Q} \sim Q^{4}$ or higher are neglected, since they renormalize higher-order counterterms. The combined effect of $\bar{K}$ and $\bar{I}$ on the loop contributions to the axial-vector form factors is shown in Fig. 3. 

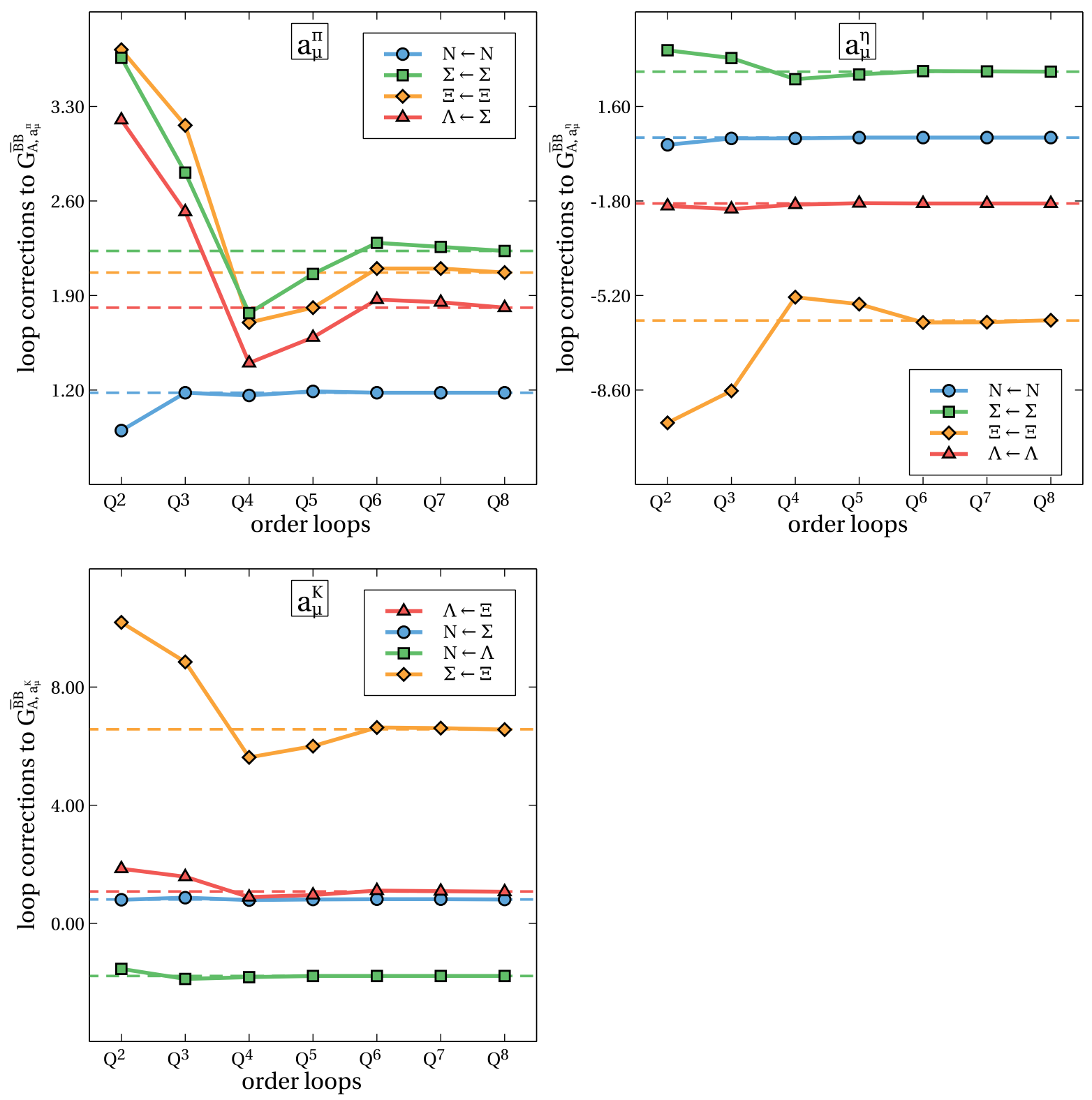

FIG. 3. Loop corrections to $G_{A, i}^{\bar{B} B}\left(t=\left(M_{\bar{p}}-M_{p}\right)^{2}\right)$ for different axial-vector currents $i$. The LECs $g^{(S)}, g^{(V)}, g^{(T)}, g^{(F)}, f^{(F)}$, and $f^{(A)}$ are not included. The points refer to the expansion to the corresponding chiral order, whereas the dashed lines show the full contributions.

The convergence properties in Fig. 3 are worse than the ones in Fig. 2. Nevertheless, our power-counting scheme is well converging. The chiral order $Q^{5}$ describes the full contributions to acceptable accuracy, whereas for some processes even lower orders are sufficient. We continue the discussion for the orders $Q^{2}-Q^{5}$ and include additional terms from the Lagrangian $\mathcal{L}^{(2)}$ in Eq. (9).

In the following we also consider the LECs of the higher order Lagrangians from Eqs. (15) and (17). Note that the LECs from the Lagrangian $\mathcal{L}^{(2)}$ directly contribute to the loop contributions, whereas the LECs from $\mathcal{L}^{(3)}$ do not interfere with the loops. At first we include the LECs $g^{(S)}$,
$g^{(V)}, g^{(T)}$, and $f^{(A)}$ from the Lagrangian $\mathcal{L}^{(2)}$, which directly contribute to the loop contributions of the order $Q^{3}$. The LECs $g^{(F)}$ and $f^{(F)}$ from Eq. (15) are not considered in this work, whereas the LECs $g^{(\chi)}$ and $g^{(R)}$ from $\mathcal{L}^{(3)}$ will be added later.

The constants with scalar and vector structure $g^{(S)}$ and $g^{(V)}$ have been determined in Ref. [11], whereas the LECs $g^{(T)}$ and $f^{(A)}$ are unknown. Since the success of Cabibbo's model [2] is compatible with small loop contributions, we determine the LECs $g^{(T)}$ and $f^{(A)}$ using a fit, which minimizes the loop contributions of all processes. This is performed for all orders $Q^{3}, Q^{4}$, and $Q^{5}$ separately, 
TABLE VIII. Left column: input LECs for the numerical estimates in flavor-SU(3) from Ref. [11]. Right column: LECs as determined by our fit from the minimization of the loop contributions of order $Q^{3}, Q^{4}$, and $Q^{5}$. The magnitude of the remaining one-loop contributions is described by the chi-square per number of processes $\chi^{2} / N_{p}$ and the maximal value max.

\begin{tabular}{lccccc}
\hline \hline LEC $\left(\mathrm{GeV}^{-1}\right)$ & From [11] & LEC & $Q^{3}$ & $Q^{4}$ & $Q^{5}$ \\
\hline$g_{0}^{(S)}$ & -8.8678 & $g_{1}^{(T)}\left(\mathrm{GeV}^{-1}\right)$ & 0.87 & 1.67 & 4.32 \\
$g_{1}^{(S)}$ & 0.8058 & $g_{D}^{(T)}\left(\mathrm{GeV}^{-1}\right)$ & -6.35 & -4.13 & -6.10 \\
$g_{D}^{(S)}$ & -1.4485 & $g_{F}^{(T)}\left(\mathrm{GeV}^{-1}\right)$ & -8.22 & -9.47 & -7.76 \\
$g_{F}^{(S)}$ & -5.1101 & $f_{1}^{(A)}\left(\mathrm{GeV}^{-1}\right)$ & 1.82 & 1.67 & 6.35 \\
$g_{0}^{(V)}$ & -0.3710 & $f_{2}^{(A)}\left(\mathrm{GeV}^{-1}\right)$ & 0.71 & 0.53 & 0.39 \\
$g_{1}^{(V)}$ & -7.2709 & $f_{3}^{(A)}\left(\mathrm{GeV}^{-1}\right)$ & -9.88 & -4.65 & -8.92 \\
$g_{D}^{(V)}$ & 10.002 & $f_{4}^{(A)}\left(\mathrm{GeV}^{-1}\right)$ & 1.14 & 0.36 & -0.34 \\
$g_{F}^{(V)}$ & -2.8688 & $\chi^{2} / N_{p}$ & 0.78 & 0.24 & 0.30 \\
& & $\max$ & 1.94 & 1.05 & 1.20 \\
\hline \hline
\end{tabular}

resulting in three sets of determined LECs, which are shown in Table VIII. The size of the minimized one-loop contributions is characterized by the chi-square per number of processes $\chi^{2} / N_{p}$, with $N_{p}=12$, and the largest remaining one-loop contribution ("max"). The LECs $g^{(T)}$ and $f^{(A)}$, determined by our fit, are reasonably small. We do not give errors, but expect some range of \pm 3 for the LECs and observe quite large deviations of the LECs for different orders. This is not surprising, since our estimate is not intended to be seen as a rigorous fit, for which, apart from accurate QCD lattice data, also the LECs $g^{(F)}, f^{(F)}, g^{(R)}$, and $g^{(\chi)}$ need to be taken into account.

In Fig. 4 we reproduce the loop contributions of orders $Q^{2}-Q^{5}$ of Fig. 3, but this time with the contributions of $g^{(S)}$, $g^{(V)}, g^{(T)}$, and $f^{(A)}$ from Table VIII. Since these LECs become relevant only at order $Q^{3}$, the order- $Q^{2}$ contributions remain unchanged compared to Fig. 3. For the contributions of order $Q^{3}, Q^{4}$, and $Q^{5}$ we find that the loop contributions are drastically reduced in all processes. We observe that contributions are most efficiently minimized at order $Q^{4}$, but also the contributions at order $Q^{5}$ and, with a few exceptions, at order $Q^{3}$ are small. Therefore, we conclude that it is possible to reduce the loop contributions to the axial-vector form factors for all processes by appropriate choices for the LECs $g^{(T)}$ and $f^{(A)}$ at all orders.

As a last step, we turn on the LECs of the counterterms of the Lagrangian $\mathcal{L}^{(3)}$ in Eq. (10). Unlike the LECs $g^{(S)}$, $g^{(V)}, g^{(T)}$, and $f^{(A)}$, the LECs $g^{(\chi)}$ and $g^{(R)}$ do not enter as loop contributions but as counterterms. We find that it is possible to further reduce the one-loop contributions with these counterterms and show the resulting LECs $g_{1-7}^{(\alpha)}$ and
$g_{D / F}^{(R)}$ in Table IX. The LECs are reasonably small, fulfilling $\left|g_{1-7}^{(\chi)}\right|<3$ and $\left|g_{D / F}^{(R)}\right|<7$. The parameters $\chi^{2} / N_{p}$ and the maximal remaining contribution $\max$ are significantly smaller than the corresponding ones without the LECs $g_{1-7}^{(\chi)}$ and $g_{D / F}^{(R)}$ in Table VIII.

\section{B. Convergence properties in the flavor-SU(3) limit}

In the previous section, we showed that the inclusion of the LECs $g^{(S)}, g^{(V)}, g^{(T)}$, and $f^{(A)}$, and additionally the LECs $g^{(R)}$ and $g^{(\chi)}$, leads to strong cancellations among the various loop contributions, when the physical masses of Table VII are used. Here we want to scrutinize whether similar effects can be observed for other on-shell baryon masses. We choose a lattice QCD ensemble in the unphysical flavor-SU(3) limit, which is characterized by $m_{u}=m_{d}=m_{s}$ [37]. This leads to equal masses of the Goldstone bosons, within the baryon octet and within the baryon decuplet. The exact masses and the wave-function renormalization factor $Z$ are given in Table X.

At first, we neglect the effect of the LECs $g^{(R)}$ and $g^{(\chi)}$. In Fig. 5 the one-loop contributions are shown. At order $Q^{2}$, the LECs $g^{(S)}, g^{(V)}, g^{(T)}$, and $f^{(A)}$ are not effective, so the loop contributions are rather large. For the higher-order terms $Q^{3}, Q^{4}$, and $Q^{5}$ the, to the relevant order corresponding, LECs $g^{(T)}$ and $f^{(A)}$ from Table VIII are included. This procedure evidently reduces the loop contributions for all processes. The resulting terms of order $Q^{3}$ and $Q^{5}$ are very small, the order- $Q^{4}$ contributions only slightly larger. We want to stress here that these contributions were not directly minimized by a fit, but only by the use of the LECs of Table VIII.

In the next step, we also include the LECs $g^{(\chi)}$ and $g^{(R)}$ from the Lagrangian $\mathcal{L}^{(3)}$. We use their values from Table IX and perform a comparison of important parameters of the one-loop contributions between the cases with and without the counterterms $g^{(R)}$ and $g^{(\chi)}$. We give the chi-square per number of processes $\chi^{2} / N_{p}$ and the largest remaining one-loop contribution in Table XI. For the orders $Q^{3}$ and $Q^{5}$ the inclusion of $g^{(\chi)}$ and $g^{(R)}$ further reduces the one-loop contribution, whereas this is not the case for the order $Q^{4}$. A reason for that could be the appearance of additional counterterms turning relevant at order $Q^{4}$.

The numerical estimates, given in this section, show good convergence effects, for both the kinematic factors $\bar{K}$ and the one-loop contributions to the axial-vector form factors in general. With estimates for the LECs $g^{(T)}, f^{(A)}$, $g^{(\chi)}$, and $g^{(R)}$ we find large cancellations of the loop effects for the chiral order $Q^{3}$ and higher. We conclude that the comparison with QCD lattice data should be performed at the order- $Q^{3}$ level. 

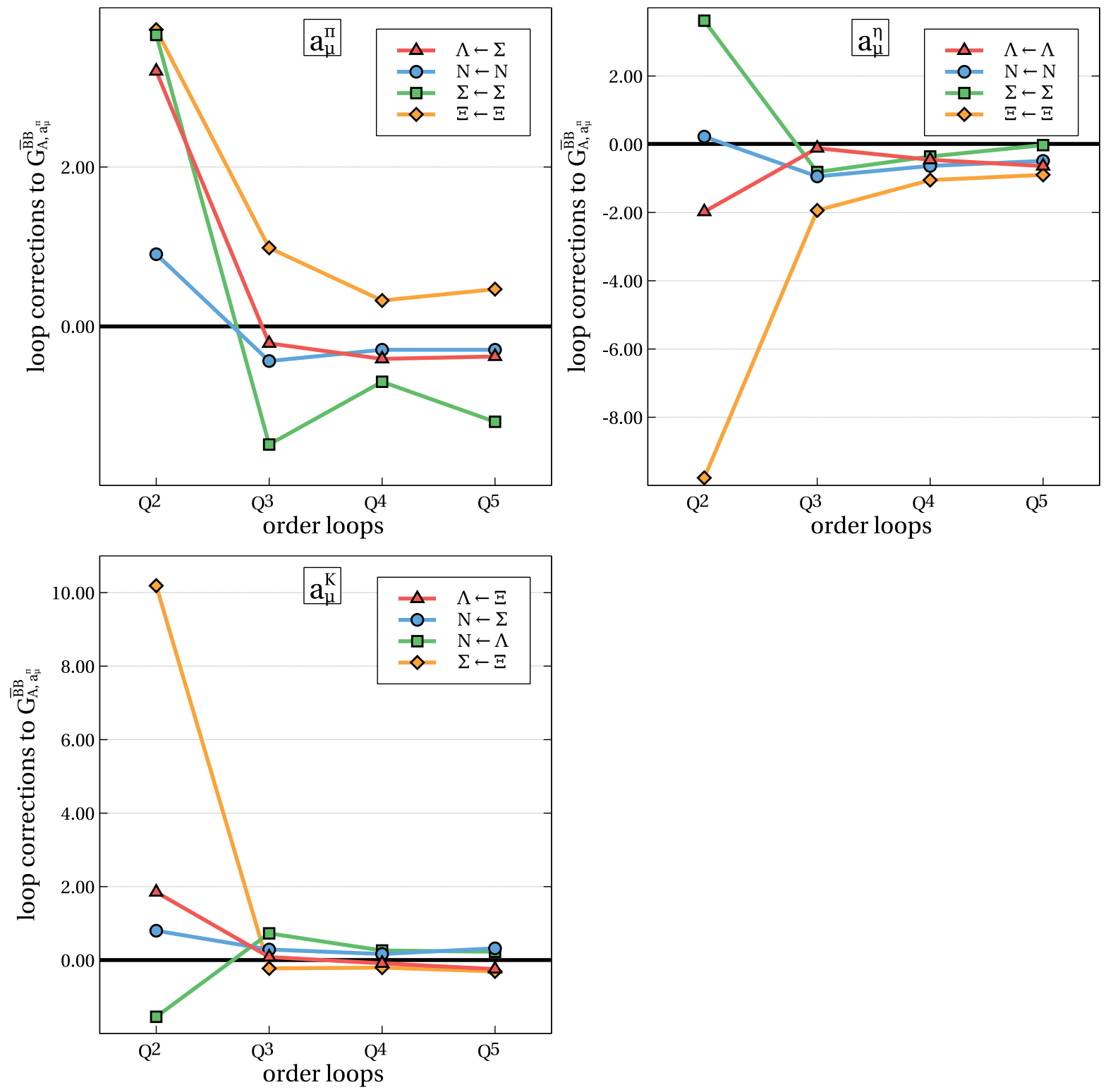

FIG. 4. Loop corrections to $G_{A, i}^{\bar{B} B}\left(t=\left(M_{\bar{p}}-M_{p}\right)^{2}\right)$ up to order $Q^{5}$ as in Fig. 3, but with the effect of the LECs $g^{(S)}, g^{(V)}, g^{(T)}$, and $f^{(A)}$ from Table VIII, which contribute to order $Q^{3}$ and higher.

\section{SUMMARY AND OUTLOOK}

TABLE IX. LECs as determined by our fit. We also give the chi-square per number of processes $\chi^{2} / N_{p}$ and the largest remaining one-loop contribution max.

\begin{tabular}{lccccccc}
\hline \hline Order & $Q^{3}$ & $Q^{4}$ & $Q^{5}$ & Order & $Q^{3}$ & $Q^{4}$ & $Q^{5}$ \\
\hline$g_{1}^{(\chi)}$ & -0.46 & -0.18 & -0.58 & $g_{5}^{(\chi)}$ & -2.44 & -1.14 & -0.25 \\
$g_{2}^{(\chi)}$ & -1.87 & -0.76 & -0.88 & $g_{6}^{(\chi)}$ & 2.43 & -0.13 & -0.29 \\
$g_{3}^{(\chi)}$ & -2.64 & -1.27 & -2.01 & $g_{7}^{(\chi)}$ & 2.27 & 1.11 & 1.86 \\
$g_{4}^{(\chi)}$ & -0.43 & -0.47 & -0.69 & $g_{D}^{(R)}$ & 6.71 & 4.38 & 6.75 \\
$\chi^{2} / N_{p}$ & 0.06 & 0.02 & 0.01 & $g_{F}^{(R)}$ & 6.84 & 3.83 & 4.94 \\
$\max$ & 0.61 & 0.35 & 0.28 & & & & \\
\hline \hline
\end{tabular}

In this paper, we investigated the axial-vector currents of the baryon octet in flavor-SU(3) chiral perturbation theory. We established analytical results for the axial-vector form factors at $\mathrm{N}^{2} \mathrm{LO}$ that include one-loop contributions. The

TABLE X. QCD lattice masses in the flavor-SU(3) limit [37].

\begin{tabular}{lc}
\hline \hline Mass & Lattice ensemble [37] \\
\hline$m_{\pi}, m_{K}, m_{\eta}(\mathrm{MeV})$ & 391 \\
$M_{N}, M_{\Lambda}, M_{\Sigma}, M_{\Xi}(\mathrm{MeV})$ & 1124 \\
$M_{\Delta_{\mu}}, M_{\Sigma_{\mu}}, M_{\Xi_{\mu}}, M_{\Omega_{\mu}}(\mathrm{MeV})$ & 1438 \\
$Z_{N}, Z_{\Lambda}, Z_{\Sigma}, Z_{\Xi}$ & 2.28 \\
\hline \hline
\end{tabular}



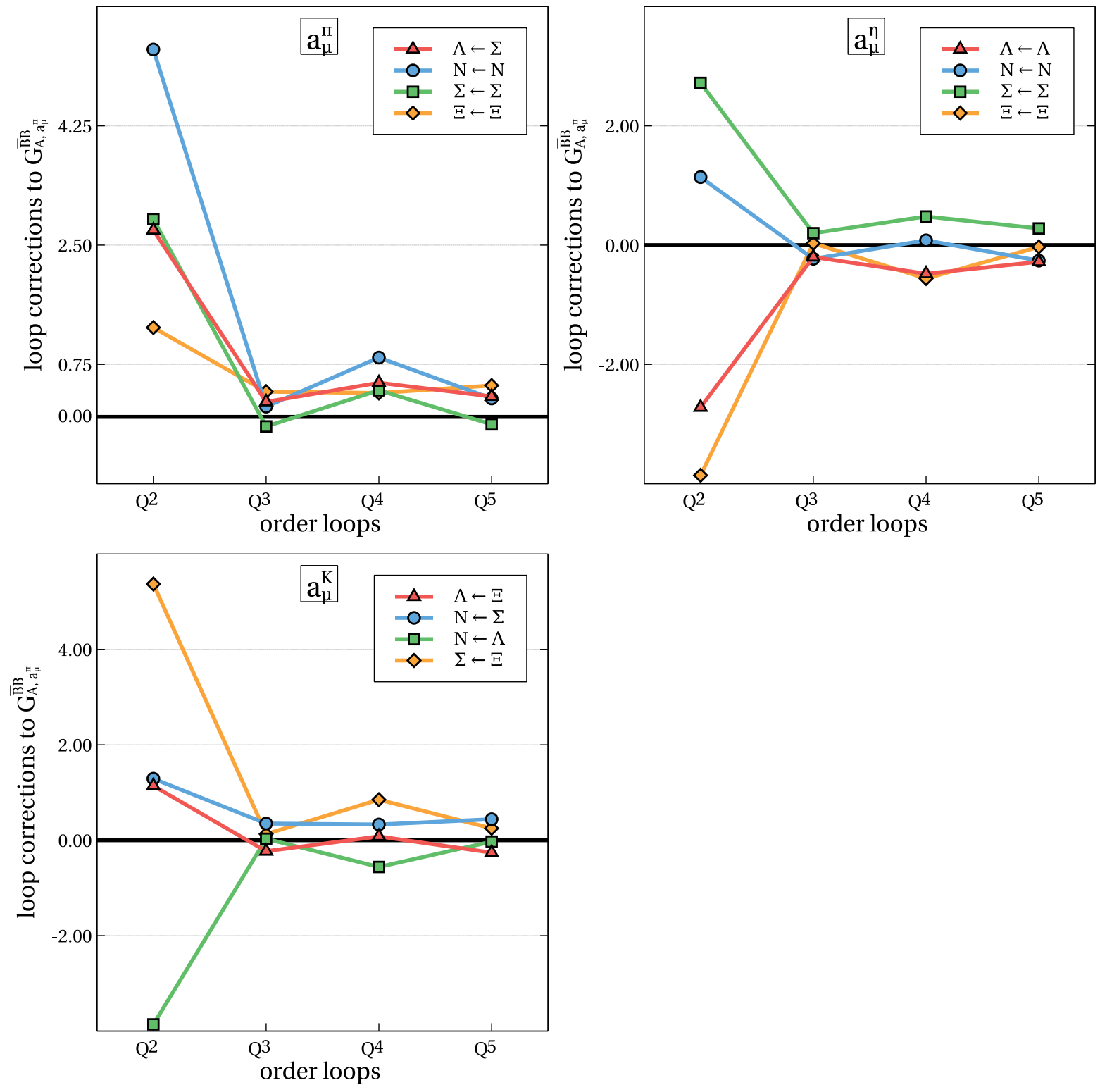

FIG. 5. Loop corrections to $G_{A, i}^{\bar{B} B}\left(t=\left(M_{\bar{p}}-M_{p}\right)^{2}\right)$ in the flavor-SU(3) limit, with the masses from Table X. The LECs $g^{(S)}, g^{(V)}, g^{(T)}$, and $f^{(A)}$ are taken from Table VIII. They are not contributing to the order $Q^{2}$. The LECs $g^{(\chi)}$ and $g^{(R)}$ are not included here.

decuplet baryons were considered as an explicit degree of freedom. Since we explored the use of on-shell masses in the one-loop contributions, particular attention was paid to a power-counting scheme that leads to good convergence

TABLE XI. Parameters which quantify the success of the reduction of one-loop contributions in the flavor-SU(3) limit, $\chi^{2} / N_{p}$, and the maximal remaining contribution max.

\begin{tabular}{ccccc}
\hline \hline & & $Q^{3}$ & $Q^{4}$ & $Q^{5}$ \\
\hline With $g_{1-7}^{(\chi)}=g_{D / F}^{(R)}=0$ & $\chi^{2} / N_{p}$ & 0.04 & 0.26 & 0.08 \\
With $g_{1-7}^{(\chi)}$ and $g_{D / F}^{(R)}$ & $\chi^{2} / N_{p}$ & 0.02 & 0.31 & 0.04 \\
from Table IX & $\max$ & 0.23 & 0.92 & 0.31 \\
\hline \hline
\end{tabular}

properties. It was demonstrated that the use of on-shell masses fulfills this condition. Furthermore, the use of onshell masses with their finite-volume dependence implies an implicit finite-volume effect of the axial-vector form factors. While our analytical results are prepared to be confronted with QCD lattice data, a rich basis of QCD lattice data is so far only available in flavor-SU(2). However, we expect that additional flavor-SU(3) QCD lattice data will be published within the next few years.

Unfortunately, for most of the flavor-SU(3) QCD lattice ensembles, which were used to fit the baryon octet and decuplet masses to high accuracy [11], axial-vector form factors are not available yet. Once the data basis is significantly enlarged further investigations are planned. Our goal is a global fit of octet and decuplet masses and the 
axial-vector form factors, as presented in Ref. [5] in flavorSU(2). The large amount of low-energy constants can be reduced by appropriate large- $N_{c}$ relations, to be established in future work.

\section{ACKNOWLEDGMENTS}

We thank Stefan Leupold for helpful discussions.

\section{APPENDIX A: AMPLITUDES}

The following formulas for $J_{\ldots}^{P}$ and $J_{\ldots}^{I}$ project onto the form factors $G_{P, i}^{\bar{B} B}(t)$ and $G_{I, i}^{\bar{B} B}(t)$ just as $J_{\ldots}^{A}$ does onto $G_{A, i}^{\bar{B} B}(t)$ (30). The form factors $G_{P, i}^{\bar{B} B}(t)$ and $G_{I, i}^{\bar{B} B}(t)$ are defined in Eq. (24):

$$
\begin{aligned}
& J_{\ldots}^{P}=\frac{M_{\bar{p}} M_{p}\left(M_{\bar{p}}+M_{p}\right)^{2}}{\sqrt{2}(d-2)\left(M_{\bar{p}} M_{p}-\bar{p} \cdot p\right)\left(M_{\bar{p}} M_{p}+\bar{p} \cdot p\right)} \operatorname{tr}\left[\gamma_{\mu} \gamma_{5} \frac{\not \not p}{2 M_{\bar{p}}} M_{\bar{p}} \ldots \frac{\not p+M_{p}}{2 M_{p}}\right] \\
& +\frac{M_{\bar{p}} M_{p}\left(M_{\bar{p}}+M_{p}\right)^{2}\left((d-1)\left(M_{\bar{p}}^{2}+M_{p}^{2}\right)+2 M_{\bar{p}} M_{p}+2(d-2) \bar{p} \cdot p\right)}{2 \sqrt{2}(d-2)\left(M_{\bar{p}} M_{p}-\bar{p} \cdot p\right)^{2}\left(M_{\bar{p}} M_{p}+\bar{p} \cdot p\right)} \\
& \times \operatorname{tr}\left[\frac{\bar{p}_{\mu}-p_{\mu}}{M_{\bar{p}}+M_{p}} \gamma_{5} \frac{\bar{p}+M_{\bar{p}}}{2 M_{\bar{p}}} J_{\ldots}^{\mu} \frac{\not p+M_{p}}{2 M_{p}}\right]+\frac{(d-1) M_{\bar{p}} M_{p}\left(M_{\bar{p}}+M_{p}\right)^{4}}{2 \sqrt{2}(d-2)\left(M_{\bar{p}} M_{p}-\bar{p} \cdot p\right)^{2}\left(M_{\bar{p}} M_{p}+\bar{p} \cdot p\right)} \\
& \times \operatorname{tr}\left[\frac{(\not p+\not p)\left(\bar{p}_{\mu}+p_{\mu}\right)}{\left(M_{\bar{p}}+M_{p}\right)^{2}} \gamma_{5} \frac{\bar{p}+M_{\bar{p}}}{2 M_{\bar{p}}} J_{\ldots} \frac{\not p+M_{p}}{2 M_{p}}\right] \text {, } \\
& J_{\ldots}^{I}=-\frac{M_{\bar{p}} M_{p}\left(M_{\bar{p}}+M_{p}\right)^{2}}{\sqrt{2}(d-2)\left(M_{\bar{p}} M_{p}-\bar{p} \cdot p\right)\left(M_{\bar{p}} M_{p}+\bar{p} \cdot p\right)} \\
& \times \operatorname{tr}\left[\gamma_{\mu} \gamma_{5} \frac{\bar{p}+M_{\bar{p}}}{2 M_{\bar{p}}} J_{\ldots}^{\mu} \frac{\not p+M_{p}}{2 M_{p}}\right]-\frac{(d-1) M_{\bar{p}} M_{p}\left(M_{\bar{p}}+M_{p}\right)^{4}}{2 \sqrt{2}(d-2)\left(M_{\bar{p}} M_{p}-\bar{p} \cdot p\right)^{2}\left(M_{\bar{p}} M_{p}+\bar{p} \cdot p\right)} \\
& \times \operatorname{tr}\left[\frac{\bar{p}_{\mu}-p_{\mu}}{M_{\bar{p}}+M_{p}} \gamma_{5} \frac{\bar{p}+M_{\bar{p}}}{2 M_{\bar{p}}} J_{\ldots}^{\mu} \frac{\not p+M_{p}}{2 M_{p}}\right]-\frac{M_{\bar{p}} M_{p}\left(M_{\bar{p}}+M_{p}\right)^{4}\left((d-1)\left(M_{\bar{p}}^{2}+M_{p}^{2}\right)-2 M_{\bar{p}} M_{p}-2(d-2) \bar{p} \cdot p\right)}{2 \sqrt{2}(d-2)\left(M_{\bar{p}}-M_{p}\right)^{2}\left(M_{\bar{p}} M_{p}-\bar{p} \cdot p\right)^{2}\left(M_{\bar{p}} M_{p}+\bar{p} \cdot p\right)} \\
& \times \operatorname{tr}\left[\frac{(\not p+\not \supset)\left(\bar{p}_{\mu}+p_{\mu}\right)}{\left(M_{\bar{p}}+M_{p}\right)^{2}} \gamma_{5} \frac{\bar{p}+M_{\bar{p}}}{2 M_{\bar{p}}} J_{\ldots} \ldots \frac{\not p+M_{p}}{2 M_{p}}\right] \text {. }
\end{aligned}
$$

In the following, we give the amplitudes of all Feynman diagrams $J_{\ldots}^{\mu}$, which enter Eq. (29) in the form of $J_{\ldots}^{A}$ after the projection with formula (30). We use the following propagators in $d$ dimensions $[16,23]$, which are derived from $\mathcal{L}_{\text {kin }}$ (5) [38]:

(i) Spin-0 Goldstone boson:

$$
D_{Q}(k)=1 /\left(k^{2}-m_{Q}^{2}+i \epsilon\right),
$$

(ii) Spin-1/2 (octet) baryon:

$$
S_{R}(k)=1 /\left(\not k-M_{R}+i \epsilon\right),
$$

(iii) Spin-3/2 (decuplet) baryon:

$$
\begin{aligned}
S_{R}^{\mu \nu}(k)= & \frac{-1}{\not k-M_{R}+i \epsilon}\left(g^{\mu \nu}-\frac{\gamma^{\mu} \gamma^{\nu}}{d-1}\right. \\
& \left.+\frac{\left(k^{\mu} \gamma^{\nu}-k^{\nu} \gamma^{\mu}\right)}{(d-1) M_{R}}-\frac{(d-2) k^{\mu} k^{\nu}}{(d-1) M_{R}^{2}}\right) .
\end{aligned}
$$

The Feynman diagrams associated with the following amplitudes are given in Tables IV-VI:

$$
J^{\mu}=\frac{1}{\sqrt{2}} \gamma^{\mu} \gamma_{5}
$$

$J^{\mu}=\frac{1}{\sqrt{2} t}\left(\gamma^{\nu} \gamma_{5}\left(-\bar{p}_{\nu} \bar{p}^{\mu}+\bar{p}^{\mu} p_{\nu}+\bar{p}_{\nu} p^{\mu}-p_{\nu} p^{\mu}\right)+q^{2} \gamma^{\mu} \gamma_{5}\right)$,

$$
J_{Q}^{\mu}=\frac{i}{\sqrt{2}} \int \frac{d^{d} l}{(2 \pi)^{d}} \gamma^{\mu} \gamma_{5} D_{Q}(l),
$$

$$
\begin{aligned}
J_{L Q}^{\mu, 1} & =-\frac{i}{\sqrt{2}} \int \frac{d^{d} l}{(2 \pi)^{d}} l \gamma_{5} S_{L}(\bar{p}-l) D_{Q}(l) \gamma^{\mu}, \\
J_{L Q}^{\mu, 2} & =-\frac{i}{\sqrt{2}} \int \frac{d^{d} l}{(2 \pi)^{d}} l \gamma_{5} S_{L}(\bar{p}-l) D_{Q}(l) l^{\mu},
\end{aligned}
$$




$$
\begin{aligned}
J_{L Q}^{\mu, 3}= & -\frac{i}{\sqrt{2}} \int \frac{d^{d} l}{(2 \pi)^{d}} l \gamma_{5} S_{L}(\bar{p}-l) D_{Q}(l) \\
& \times\left(\left(\gamma^{\mu} p \cdot l+l p^{\mu}\right)+\left(\gamma^{\mu}(\bar{p}-l) \cdot l+l(\bar{p}-l)^{\mu}\right)\right) \\
J_{L Q}^{\mu, 4}= & -\frac{i}{\sqrt{2}} \int \frac{d^{d} l}{(2 \pi)^{d}} l \gamma_{5} S_{L}(\bar{p}-l) D_{Q}(l) i \sigma^{\mu \nu} l_{\nu} \\
J_{L Q}^{\mu, 5}= & \frac{i}{\sqrt{2}} \int \frac{d^{d} l}{(2 \pi)^{d}} l \gamma_{5} S_{L}(\bar{p}-l) D_{Q}(l) i \sigma^{\mu \nu}(\bar{p}-p)_{\nu} \\
J_{Q R}^{\mu, 1}= & \frac{i}{\sqrt{2}} \int \frac{d^{d} l}{(2 \pi)^{d}} \gamma^{\mu} D_{Q}(l) S_{R}(p-l) l \gamma_{5} \\
J_{Q R}^{\mu, 2}= & -\frac{i}{\sqrt{2}} \int \frac{d^{d} l}{(2 \pi)^{d}} l^{\mu} D_{Q}(l) S_{R}(p-l) l \gamma_{5} \\
J_{Q R}^{\mu, 5}= & -\frac{i}{\sqrt{2}} \int \frac{d^{d} l}{(2 \pi)^{d}} i \sigma^{\mu \nu}(\bar{p}-p)_{\nu} D_{Q}(l) S_{R}(p-l) l \gamma_{5} \\
J_{Q R}^{\mu, 4}= & -\frac{i}{\sqrt{2}} \int \frac{d^{d} l}{(2 \pi)^{d}} i \sigma^{\mu \nu} l_{\nu} D_{Q}(l) S_{R}(p-l) l \gamma_{5} \\
J_{Q R}^{\mu, 3}= & -\frac{J_{L Q}^{\mu, 1}}{\sqrt{2}} \int \frac{d^{d} l}{(2 \pi)^{d}}\left(\left(l \cdot(p-l) \gamma^{\mu}+l(p-l)^{\mu}\right)\right. \\
& \left.+\left(\gamma^{\mu} \bar{p} \cdot l+l \bar{p}^{\mu}\right)\right) D_{Q}(l) S_{R}(p-l) l \gamma_{5} \\
& \\
&
\end{aligned}
$$$$
J_{L Q}^{\mu, 2}=\frac{i}{\sqrt{2}} \int \frac{d^{d} l}{(2 \pi)^{d}} l_{\nu}\left(S_{L}^{\nu \mu}(\bar{p}-l) l-S_{L}^{\nu \alpha}(\bar{p}-l) l_{\alpha} \gamma^{\mu}\right) \gamma_{5} D_{Q}(l),
$$

$$
\begin{aligned}
& J_{L Q}^{\mu, 3}= \frac{i}{\sqrt{2}} \int \frac{d^{d} l}{(2 \pi)^{d}} l_{\nu}\left(S_{L}^{\nu \alpha}(\bar{p}-l) \gamma^{\mu}\right. \\
&\left.-S_{L}^{\nu \mu}(\bar{p}-l) \gamma^{\alpha}\right)(\bar{p}-p)_{\alpha} \gamma_{5} D_{Q}(l), \\
& J_{L Q}^{\mu, 4}=J_{L Q}^{\mu, 5}=0, \\
& J_{Q R}^{\mu, 1}=\frac{i}{\sqrt{2}} \int \frac{d^{d} l}{(2 \pi)^{d}}\left(l \gamma_{5} S_{R}^{\mu \nu}(p-l)\right. \\
&\left.+\gamma^{\mu} \gamma_{5} l_{\alpha} S_{R}^{\alpha \nu}(p-l)\right) l_{\nu} D_{Q}(l), \\
& J_{Q R}^{\mu, 2}= \frac{i}{\sqrt{2}} \int \frac{d^{d} l}{(2 \pi)^{d}}\left(l \gamma_{5} S_{R}^{\mu \nu}(p-l)\right. \\
&\left.-\gamma^{\mu} \gamma_{5} l_{\alpha} S_{R}^{\alpha \nu}(p-l)\right) l_{\nu} D_{Q}(l),
\end{aligned}
$$

$$
\begin{aligned}
J_{Q R}^{\mu, 3}= & \frac{i}{\sqrt{2}} \int \frac{d^{d} l}{(2 \pi)^{d}} \gamma_{5}(\bar{p}-p)_{\alpha}\left(\gamma^{\alpha} S_{R}^{\mu \nu}(p-l)\right. \\
& \left.-\gamma^{\mu} S_{R}^{\alpha \nu}(p-l)\right) l_{\nu} D_{Q}(l), \\
J_{Q R}^{\mu, 4} & =J_{Q R}^{\mu, 5}=0,
\end{aligned}
$$$$
J_{L Q R}^{\mu, 1}=\frac{i}{\sqrt{2}} \int \frac{d^{d} l}{(2 \pi)^{d}} l \gamma_{5} S_{L}(\bar{p}-l) \gamma^{\mu} \gamma_{5} D_{Q}(l) S_{R}(p-l) l \gamma_{5} \text {, }
$$$$
J_{L Q R}^{\mu, 1}=\frac{i}{\sqrt{2}} \int \frac{d^{d} l}{(2 \pi)^{d}} l_{\sigma} S_{L}^{\sigma \mu}(\bar{p}-l) D_{Q}(l) S_{R}(p-l) l \gamma_{5},
$$

$$
J_{L Q R}^{\mu, 1}=\frac{i}{\sqrt{2}} \int \frac{d^{d} l}{(2 \pi)^{d}} l \gamma_{5} S_{L}(\bar{p}-l) D_{Q}(l) S_{R}^{\mu \tau}(p-l) l_{\tau},
$$

$$
J_{L Q R}^{\mu, 1}=\frac{i}{\sqrt{2}} \int \frac{d^{d} l}{(2 \pi)^{d}} l_{\sigma} S_{L}^{\sigma \alpha}(\bar{p}-l) D_{Q}(l) \gamma^{\mu} \gamma_{5} S_{\alpha \beta}^{R}(p-l) l^{\beta}
$$

$$
\begin{aligned}
J_{L Q R}^{\mu, 2}= & \frac{i}{\sqrt{2}} \int \frac{d^{d} l}{(2 \pi)^{d}} l_{\sigma}\left(S_{L}^{\sigma \alpha}(\bar{p}-l) \gamma^{\mu}\right. \\
& \left.-S_{L}^{\sigma \mu}(\bar{p}-l) \gamma^{\alpha}\right)(\bar{p}-p)_{\alpha} D_{Q}(l) S_{R}(p-l) l \gamma_{5}, \\
J_{L Q R}^{\mu, 2}= & \frac{i}{\sqrt{2}} \int \frac{d^{d} l}{(2 \pi)^{d}} l \gamma_{5} S_{L}(\bar{p}-l) D_{Q}(l)\left(\gamma^{\alpha} S_{R}^{\mu \tau}(p-l)\right. \\
& \left.-\gamma^{\mu} S_{R}^{\alpha \tau}(p-l)\right)(\bar{p}-p)_{\alpha} l_{\tau} .
\end{aligned}
$$

\section{APPENDIX B: CGCS WITHOUT AXIAL-VECTOR CURRENT}

In this part we give the 3-point Clebsch-Gordan coefficients $G_{Q R}^{(B)}$ with the Goldstone boson $Q$ and the baryons $B, R \in[8],[10]$.

\section{APPENDIX C: CGCS OF COUNTERTERMS}

Here we give the CGCs of the counterterms $X_{i}^{(\bar{B} B)}$ and $T_{i}^{(\bar{B} B)}$.

\section{APPENDIX D: CGCS WITH AXIAL-VECTOR CURRENT}

In this section we show all CGCs $A$ with axial-vector current, the 3-point coefficients in Tables XVIII-XXI, the 4-point coefficients in Tables XXIII-XXVII, and the 5-point CGCs in Table XXII. 
TABLE XII. CGCs $G_{Q R}^{(B)}$ with $B, R \in[8]$.

\begin{tabular}{lll}
\hline \hline$G_{\pi N}^{(N)}=\sqrt{3}(D+F)$ & $G_{\pi \Sigma}^{(\Lambda)}=2 D$ & $G_{\pi \Lambda}^{(\Sigma)}=\frac{2}{\sqrt{3}} D$ \\
$G_{\eta N}^{(N)}=-\frac{1}{\sqrt{3}}(D-3 F)$ & $G_{\overline{K N}}^{(\Lambda)}=-\sqrt{\frac{2}{3}}(D+3 F)$ & $G_{\pi \Sigma}^{(\Sigma)}=-2 \sqrt{2} F$ \\
$G_{K \Lambda}^{(N)}=-\frac{1}{\sqrt{3}}(D+3 F)$ & $G_{\eta \Lambda}^{(\Lambda)}=-\frac{2}{\sqrt{3}} D$ & $G_{\overline{K N}}^{(\Sigma)}=\sqrt{2}(D-F)$ \\
$G_{K \Sigma}^{(N)}=\sqrt{3}(D-F)$ & $G_{K \Xi}^{(\Lambda)}=\sqrt{\frac{2}{3}}(D-3 F)$ & $G_{\eta \Sigma}^{(\Sigma)}=\frac{2}{\sqrt{3}} D$ \\
$G_{\pi \Xi}^{(\Xi)}=-\sqrt{3}(D-F)$ & $G_{\overline{K \Lambda}}^{(\Xi)}=-\frac{1}{\sqrt{3}}(D-3 F)$ & $G_{K \Xi}^{(\Sigma)}=\sqrt{2}(D+F)$ \\
$G_{\overline{K \Sigma}}^{(\Xi)}=-\sqrt{3}(D+F)$ & $G_{\eta \Xi}^{(\Xi)}=-\frac{1}{\sqrt{3}}(D+3 F)$ & \\
\hline \hline
\end{tabular}

TABLE XIII. CGCs $G_{Q R}^{(B)}$ with $B \in[10]$, denoted by $\mu$, and $R \in[8]$.

\begin{tabular}{llll}
\hline \hline$G_{\pi N}^{\left(\Delta_{\mu}\right)}=\sqrt{2} C$ & $G_{\pi \Lambda}^{\left(\Sigma_{\mu}\right)}=-C$ & $G_{\pi \Xi}^{\left(\Xi_{\mu}\right)}=-C$ & $G_{\bar{K} \Xi}^{\left(\Omega_{\mu}\right)}=-2 C$ \\
$G_{K \Sigma}^{\left(\Delta_{\mu}\right)}=-\sqrt{2} C$ & $G_{\pi \Sigma}^{\left(\Sigma_{\mu}\right)}=-\sqrt{\frac{2}{3} C}$ & $G_{\bar{K} \Lambda}^{\left(\Xi_{\mu}\right)}=C$ & \\
$G_{\bar{K} N}^{\left(\Sigma_{\mu}\right)}=\sqrt{\frac{2}{3}} C$ & $G_{\bar{K} \Sigma}^{\left(\Xi_{\mu}\right)}=C$ & $G_{\eta \Xi}^{\left(\Xi_{\mu}\right)}=-C$ & \\
$G_{\eta \Sigma}^{\left(\Sigma_{\mu}\right)}=C$ & & \\
$G_{K \Xi}^{\left(\Sigma_{\mu}\right)}$ & $=-\sqrt{\frac{2}{3} C}$ & & \\
\hline \hline
\end{tabular}

TABLE XIV. CGCs $G_{Q R}^{(B)}$ with $B \in[8]$ and $R \in[10]$, denoted by $\mu$.

\begin{tabular}{llll}
\hline \hline$G_{\pi \Delta_{\mu}}^{(N)}=2 C$ & $G_{\pi \Sigma_{\mu}}^{(\Lambda)}=-\sqrt{3} C$ & $G_{\pi \Sigma_{\mu}}^{(\Sigma)}=-\sqrt{\frac{2}{3}} C$ & $G_{\pi \Xi_{\mu}}^{(\Xi)}=-C$ \\
$G_{K \Sigma_{\mu}}^{(N)}=C$ & $G_{K \Xi_{\mu}}^{(\Lambda)}=-\sqrt{2} C$ & $G_{\bar{K} \Delta_{\mu}}^{(\Sigma)}=-\sqrt{\frac{8}{3}} C$ & $G_{\eta \Xi_{\mu}}^{(\Xi)}=-C$ \\
& $G_{\eta \Sigma_{\mu}}^{(\Sigma)}=C$ & $G_{\bar{K} \Sigma_{\mu}}^{(\Xi)}=C$ \\
& $G_{K \Xi_{\mu}}^{(\Sigma)}=-\sqrt{\frac{2}{3} C}$ & $G_{K \Omega_{\mu}}^{(\Xi)}=-\sqrt{2} C$ \\
\hline \hline
\end{tabular}

TABLE XV. CGCs $G_{Q R}^{(B)}$ with $B, R \in[10]$, denoted by $\mu$.

\begin{tabular}{llll}
\hline \hline$G_{\pi \Delta_{\mu}}^{\left(\Delta_{\mu}\right)}=-\sqrt{\frac{5}{3}} H$ & $G_{\pi \Sigma_{\mu}}^{\left(\Sigma_{\mu}\right)}=\frac{2 \sqrt{2}}{3} H$ & $G_{\pi \Xi_{\mu}}^{\left(\Xi_{\mu}\right)}=-\frac{1}{\sqrt{3}} H$ & $G_{\bar{K} \Xi_{\mu}}^{\left(\Omega_{\mu}\right)}=-\frac{2}{\sqrt{3}} H$ \\
$G_{\eta \Delta_{\mu}}^{\left(\Delta_{\mu}\right)}=-\frac{1}{\sqrt{3}} H$ & $G_{\left.\bar{K} \Delta_{\mu}\right)}^{\left(\Sigma_{\mu}\right)}=-\frac{2 \sqrt{2}}{3} H$ & $G_{\bar{K} \Sigma_{\mu}}^{\left(\Xi_{\mu}\right)}=-\frac{2}{\sqrt{3}} H$ & $G_{\eta \Omega_{\mu}}^{\left(\Omega_{\mu}\right)}=\frac{2}{\sqrt{3}} H$ \\
$G_{K \Sigma_{\mu}}^{\left(\Delta_{\mu}\right)}=-\sqrt{\frac{2}{3}} H$ & $G_{\eta \Sigma_{\mu}}^{\left(\Sigma_{\mu}\right)}=0$ & $G_{K \Omega_{\mu}}^{\left(\Xi_{\mu}\right)}=-\sqrt{\frac{2}{3}} H$ & \\
& $G_{K \Xi_{\mu}}^{\left(\Sigma_{\mu}\right)}=\frac{2 \sqrt{2}}{3} H$ & $G_{\eta \Xi_{\mu}}^{\left(\Xi_{\mu}\right)}=\frac{1}{\sqrt{3}} H$ & \\
\hline \hline
\end{tabular}

The 4-point vector couplings $A_{Q L,(\bar{B})}^{(i B), 3}$ are given by

$$
A_{Q L \in[8],(\bar{B})}^{(i B), 3}=\frac{1}{4} A_{Q L \in[8],(\bar{B})}^{(i B), 1} \quad \text { with } \quad g_{X}^{(S)} \rightarrow g_{X}^{(V)} .
$$

Furthermore, the parameter $n$ of the sum in Eq. (29) for $L \in[10]$ only leads to nonvanishing contributions up to $n=2$, so

$$
A_{Q L \in[10],(\bar{B})}^{(i B), 3}=A_{Q L \in[10],(\bar{B})}^{(i B), 4}=0 .
$$

For the bubble diagram with internal baryon $R$ in Eq. (29) the 4-point vertices $A_{Q R,(B)}^{(i \bar{B}), n}$ can be extracted from $A_{Q L,(\bar{B})}^{(i B), n}$ by

$$
\begin{array}{rr}
A_{Q R \in[8],(B)}^{(i \bar{B}), 1}=-A_{Q L \in[8],(\bar{B})}^{(\tilde{i} B), 1}, & A_{Q R \in[8],(B)}^{(i \bar{B}), 2}=A_{Q L \in[8],(\bar{B})}^{(\bar{i} B),}, \\
A_{Q R \in[8],(B)}^{(i \bar{B}), 3}=A_{Q L \in[8],(\bar{B})}^{(\tilde{i}), 3}, & A_{Q R \in[8],(B)}^{(i \bar{B}), 4}=-A_{Q L \in[8],(\bar{B})}^{(\bar{i} B),}, \\
A_{Q R \in[10],(B)}^{(i \bar{B}), 1}=A_{Q L \in[10],(\bar{B})}^{(\tilde{i} B), 1}, & A_{Q R \in[10],(B)}^{(i \bar{B}), 2}=A_{Q L \in[10],(\bar{B})}^{(\tilde{i} B), 2}, \\
A_{Q R \in[10],(B)}^{(i \bar{B}), 3}=A_{Q R \in[10],(B)}^{(i \bar{B}), 4}=0 . & \text { (D3) }
\end{array}
$$

Note that the axial-vector current has to be turned around; in other words, we have to replace $a_{\mu}^{\bar{K}} \leftrightarrow a_{\mu}^{K}$, but the contributions of $a_{\mu}^{\pi}$ and $a_{\mu}^{\eta}$ remain unchanged: 
TABLE XVI. CGCs $X_{i}^{(\bar{B} B)}$ with the axial-vector current $i$ and the baryons $\bar{B}, B \in[8]$.

$X_{a_{\mu}^{n}}^{(N N)}=\frac{1}{\sqrt{6}}\left(-g_{1}^{(\chi)}-g_{2}^{(\chi)}+3 g_{3}^{(\chi)}+3 g_{4}^{(\chi)}+2 g_{5}^{(\chi)}+6 g_{7}^{(\chi)}\right) m+\frac{1}{\sqrt{6}}\left(-g_{1}^{(\chi)}+g_{2}^{(\chi)}+3 g_{3}^{(\chi)}-3 g_{4}^{(\chi)}-2 g_{5}^{(\chi)}+3 g_{7}^{(\chi)}\right) m_{s}$

$X_{a_{\mu}^{\eta}}^{(\Sigma \Sigma)}=\sqrt{\frac{2}{3}}\left(2 g_{1}^{(\chi)}+g_{5}^{(\chi)}\right) m-\sqrt{\frac{2}{3}} g_{5}^{(\chi)} m_{s}$

$X_{a_{\mu}^{\eta}}^{(\Xi \Xi)}=-\frac{1}{\sqrt{6}}\left(g_{1}^{(\chi)}-g_{2}^{(\chi)}+3 g_{3}^{(\chi)}-3 g_{4}^{(\chi)}-2 g_{5}^{(\chi)}+6 g_{7}^{(\chi)}\right) m-\frac{1}{\sqrt{6}}\left(g_{1}^{(\chi)}+g_{2}^{(\chi)}+3 g_{3}^{(\chi)}+3 g_{4}^{(\chi)}+2 g_{5}^{(\chi)}+3 g_{7}^{(\chi)}\right) m_{s}$

$X_{a_{\mu}^{\eta}}^{(\Lambda \Lambda)}=\frac{\sqrt{2}}{3 \sqrt{3}}\left(2 g_{1}^{(\chi)}+3 g_{5}^{(\chi)}+3 g_{6}^{(\chi)}\right) m-\frac{\sqrt{2}}{3 \sqrt{3}}\left(8 g_{1}^{(\chi)}+3 g_{5}^{(\chi)}+3 g_{6}^{(\chi)}\right) m_{s}$

$X_{a_{\mu}^{\pi}}^{(N N)}=\left(g_{1}^{(\chi)}+g_{2}^{(\chi)}+g_{3}^{(\chi)}+g_{4}^{(\chi)}+2 g_{7}^{(\chi)}\right) m+\left(g_{1}^{(\chi)}-g_{2}^{(\chi)}+g_{3}^{(\chi)}-g_{4}^{(\chi)}+g_{7}^{(\chi)}\right) m_{s}$

$X_{a_{\mu}^{\pi}}^{(\Sigma \Sigma)}=2 \sqrt{2}\left(g_{3}^{(\chi)}+g_{7}^{(\chi)}\right) m+\sqrt{2} g_{7}^{(\chi)} m_{s}$

$X_{a_{\mu}^{\pi}}^{(\Xi \Xi)}=\left(g_{1}^{(\chi)}-g_{2}^{(\chi)}-g_{3}^{(\chi)}+g_{4}^{(\chi)}-2 g_{7}^{(\chi)}\right) m\left(g_{1}^{(\chi)}+g_{2}^{(\chi)}-g_{3}^{(\chi)}-g_{4}^{(\chi)}-g_{7}^{(\chi)}\right) m_{s}$

$X_{a_{\mu}^{\pi}}^{(\Sigma \Lambda)}=X_{a_{\mu}^{\pi}}^{(\Lambda \Sigma)}=\frac{1}{\sqrt{6}}\left(4 g_{1}^{(\chi)}+g_{6}^{(\chi)}\right) m-\frac{1}{\sqrt{6}} g_{6}^{(\chi)} m_{s}$

$X_{a_{\mu}^{K}}^{(N \Sigma)}=X_{a_{\mu}^{\bar{K}}}^{(\Sigma N)}=\frac{1}{2}\left(3 g_{1}^{(\chi)}+g_{2}^{(\chi)}-3 g_{3}^{(\chi)}-g_{4}^{(\chi)}-4 g_{7}^{(\chi)}\right) m+\frac{1}{2}\left(g_{1}^{(\chi)}-g_{2}^{(\chi)}-g_{3}^{(\chi)}+g_{4}^{(\chi)}-2 g_{7}^{(\chi)}\right) m_{s}$

$X_{a_{\mu}^{K}}^{(N \Lambda)}=X_{a_{\mu}^{\bar{K}}}^{(\Lambda N)}=-\frac{1}{2 \sqrt{6}}\left(-g_{1}^{(\chi)}+g_{2}^{(\chi)}+5 g_{3}^{(\chi)}+3 g_{4}^{(\chi)}-2 g_{6}^{(\chi)}+12 g_{7}^{(\chi)}\right) m-\frac{1}{2 \sqrt{6}}\left(5 g_{1}^{(\chi)}-g_{2}^{(\chi)}+7 g_{3}^{(\chi)}-3 g_{4}^{(\chi)}+2 g_{6}^{(\chi)}+6 g_{7}^{(\chi)}\right) m_{s}$

$X_{a_{\mu}^{K}}^{(\Sigma \Xi)}=X_{a_{\mu}^{\bar{K}}}^{(\Xi \Sigma)}=\frac{1}{2}\left(3 g_{1}^{(\chi)}-g_{2}^{(\chi)}+3 g_{3}^{(\chi)}-g_{4}^{(\chi)}+4 g_{7}^{(\chi)}\right) m+\frac{1}{2}\left(g_{1}^{(\chi)}+g_{2}^{(\chi)}+g_{3}^{(\chi)}+g_{4}^{(\chi)}+2 g_{7}^{(\chi)}\right) m_{s}$

$X_{a_{\mu}^{K}}^{(\Lambda \Xi)}=X_{a_{\mu}^{K}}^{(\Xi \Lambda)}=\frac{1}{2 \sqrt{6}}\left(g_{1}^{(\chi)}+g_{2}^{(\chi)}+5 g_{3}^{(\chi)}-3 g_{4}^{(\chi)}+2 g_{6}^{(\chi)}+12 g_{7}^{(\chi)}\right) m+\frac{1}{2 \sqrt{6}}\left(-5 g_{1}^{(\chi)}-g_{2}^{(\chi)}+7 g_{3}^{(\chi)}+3 g_{4}^{(\chi)}-2 g_{6}^{(\chi)}+6 g_{7}^{(\alpha)}\right) m_{s}$

TABLE XVII. CGCs $T_{i}^{(\bar{B} B)}$ with the axial-vector current $i$ and the baryons $\bar{B}, B \in[8]$.

\begin{tabular}{ll}
\hline$T_{a_{\mu}^{n}}^{(N N)}=-\sqrt{\frac{2}{3}}\left(g_{D}^{(R)}-3 g_{F}^{(R)}\right)$ & $T_{a_{\mu}^{n}}^{(\Sigma \Sigma)}=2 \sqrt{\frac{2}{3}} g_{D}^{(R)}$ \\
$T_{a_{\mu}^{(\Xi \Xi)}}^{(\Sigma \Sigma)}=-\sqrt{\frac{2}{3}}\left(g_{D}^{(R)}+3 g_{F}^{(R)}\right)$ & $T_{a_{\mu}^{n}}^{(\Lambda \Lambda)}=-2 \sqrt{\frac{2}{3}} g_{D}^{(R)}$ \\
$T_{a_{\mu}^{\pi}}^{(N N)}=2\left(g_{D}^{(R)}+g_{F}^{(R)}\right)$ & $T_{a_{\mu}^{\pi}}^{(\Sigma \Sigma)}=2 \sqrt{2} g_{F}^{(R)}$ \\
$T_{a_{\mu}^{(I \Sigma)}}^{(\Sigma \Sigma)}=2\left(g_{D}^{(R)}-g_{F}^{(R)}\right)$ & $T_{a_{\mu}^{\pi}}^{(\Sigma \Lambda)}=T_{a_{\mu}^{\pi}}^{(\Lambda \Sigma)}=2 \sqrt{\frac{2}{3}} g_{D}^{(R)}$ \\
$T_{a_{\mu}^{K}}^{(N \Sigma)}=T_{a_{\mu}^{K}}^{(\Sigma N)}=2\left(g_{D}^{(R)}-g_{F}^{(R)}\right)$ & $T_{a_{\mu}^{K}}^{(N \Lambda)}=T_{a_{\mu}^{K}}^{(\Lambda N)}=-\sqrt{\frac{2}{3}}\left(g_{D}^{(R)}+3 g_{F}^{(R)}\right)$ \\
$T_{a_{\mu}^{K}}^{(\Sigma \Xi)}=T_{a_{\mu}^{K}}^{(\Xi \Sigma)}=2\left(g_{D}^{(R)}+g_{F}^{(R)}\right)$ & $T_{a_{\mu}^{K}}^{(\Lambda \Xi)}=T_{a_{\mu}^{K}}^{(\Xi \Lambda)}=-\sqrt{\frac{2}{3}}\left(g_{D}^{(R)}-3 g_{F}^{(R)}\right)$ \\
\hline \hline
\end{tabular}

TABLE XVIII. CGCs $A_{\bar{B} B}^{(i)}$ with axial-vector current $i$ and $\bar{B}, B \in[8]$.

\begin{tabular}{lll}
\hline \hline$A_{N N}^{\left(a_{\mu}^{n}\right)}=-\frac{1}{\sqrt{6}}(D-3 F)$ & $A_{N N}^{\left(a_{\mu}^{\pi}\right)}=D+F$ & $A_{N \Sigma}^{\left(a_{\mu}^{K}\right)}=A_{\Sigma N}^{\left(a_{\mu}^{\bar{K}}\right)}=D-F$ \\
$A_{\Sigma \Sigma}^{\left(a_{\mu}^{n}\right)}=\sqrt{\frac{2}{3}} D$ & $A_{\Sigma \Sigma}^{\left(a_{\mu}^{\pi}\right)}=\sqrt{2} F$ & $A_{N \Lambda}^{\left(a_{\mu}^{K}\right)}=A_{\Lambda N}^{\left(a_{\mu}^{\bar{K}}\right)}=-\frac{1}{\sqrt{6}}(D+3 F)$ \\
$A_{\Xi \Xi}^{\left(a_{\mu}^{n}\right)}=-\frac{1}{\sqrt{6}}(D+3 F)$ & $A_{\Xi \Xi}^{\left(a_{\mu}^{\pi}\right)}=D-F$ & $A_{\Sigma \Xi}^{\left(a_{\mu}^{K}\right)}=A_{\Xi \Sigma}^{\left(a_{\mu}^{\bar{K}}\right)}=D+F$ \\
$A_{\Lambda \Lambda}^{\left(a_{\mu}^{n}\right)}=-\sqrt{\frac{2}{3}} D$ & $A_{\Sigma \Lambda}^{\left(a_{\mu}^{\pi}\right)}=\sqrt{\frac{2}{3}} D$ & $A_{\Lambda \Xi}^{\left(a_{\mu}^{K}\right)}=A_{\Xi \Lambda}^{\left(a_{\mu}^{\bar{K}}\right)}=-\frac{1}{\sqrt{6}}(D-3 F)$ \\
& $A_{\Lambda \Sigma}^{\left(a_{\mu}^{\pi}\right)}=\sqrt{\frac{2}{3}} D$ & \\
\hline \hline
\end{tabular}


TABLE XIX. CGCs $A_{\bar{B} B}^{(i)}$ with axial-vector current $i, \bar{B} \in[10]$, denoted by $\mu$, and $B \in[8]$.

\begin{tabular}{|c|c|c|c|}
\hline$A_{\Sigma_{\mu} \Sigma}^{\left(a_{\mu}^{\prime}\right)}=\frac{1}{\sqrt{2}} C$ & $A_{\Delta_{\mu} N}^{\left(a_{\mu}^{\pi}\right)}=C$ & $A_{\Delta_{\mu} \Sigma}^{\left(a_{\mu}^{K}\right)}=-\frac{1}{\sqrt{3}} C$ & $A_{\Sigma_{\mu} N}^{\left(a_{\mu}^{\bar{K}}\right)}=\frac{1}{\sqrt{3}} C$ \\
\hline \multirow[t]{3}{*}{$A_{\Xi_{\mu} \Xi}^{\left(a_{\mu}^{\eta}\right)}=-\frac{1}{\sqrt{2}} C$} & $A_{\Sigma_{\mu} \Sigma}^{\left(a_{\mu}^{\pi}\right)}=\frac{1}{\sqrt{6}} C$ & $A_{\Sigma_{\mu} \Xi}^{\left(a_{\mu}^{K}\right)}=-\frac{1}{\sqrt{3}} C$ & $A_{\Xi_{\mu} \Sigma}^{\left(a_{\mu}^{\bar{K}}\right)}=-\frac{1}{\sqrt{3}} C$ \\
\hline & $A_{\Xi_{\mu} \Xi}^{\left(a_{\mu}^{\pi}\right)}=\frac{1}{\sqrt{3}} C$ & & $A_{\Xi_{\mu} \Lambda}^{\left(a_{\mu}^{\bar{K}}\right)}=\frac{1}{\sqrt{2}} C$ \\
\hline & $A_{\Sigma_{\mu} \Lambda}^{\left(a_{\mu}^{\pi}\right)}=-\frac{1}{\sqrt{2}} C$ & & $A_{\Omega_{\mu} \Xi}^{\left(a_{\mu}^{\bar{K}}\right)}=-C$ \\
\hline
\end{tabular}

TABLE XX. CGCs $A_{\bar{B} B}^{(i)}$ with axial-vector current $i, \bar{B} \in[8]$ and $B \in[10]$, denoted by $\mu$.

\begin{tabular}{|c|c|c|c|}
\hline$A_{\Sigma \Sigma_{\mu}}^{\left(a_{\mu}^{n}\right)}=\frac{1}{\sqrt{2}} C$ & $A_{N \Delta_{\mu}}^{\left(a_{\mu}^{\pi}\right)}=C$ & $A_{N \Sigma_{\mu}}^{\left(a_{\mu}^{K}\right)}=\frac{1}{\sqrt{3}} C$ & $A_{\Sigma \Delta_{\mu}}^{\left(a_{\mu}^{\bar{K}}\right)}=-\frac{1}{\sqrt{3}} C$ \\
\hline \multirow[t]{3}{*}{$A_{\Xi \Xi}^{\left(a_{\mu}^{\eta}\right)}=-\frac{1}{\sqrt{2}} C$} & $A_{\Sigma \Sigma_{\mu}}^{\left(a_{\mu}^{\pi}\right)}=\frac{1}{\sqrt{6}} C$ & $A_{\Sigma \Xi_{\mu}}^{\left(a_{\mu}^{K}\right)}=-\frac{1}{\sqrt{3}} C$ & $A_{\Xi \Sigma_{\mu}}^{\left(a_{\mu}^{\tilde{K}}\right)}=-\frac{1}{\sqrt{3}} C$ \\
\hline & $A_{\Xi \Xi}^{\left(a_{\mu}^{T}\right)}=\frac{1}{\sqrt{3}} C$ & $A_{\Lambda \Xi_{\mu}}^{\left(a_{\mu}^{K}\right)}=\frac{1}{\sqrt{2}} C$ & \\
\hline & $A_{\Lambda \Sigma_{\mu}}^{\left(a_{\mu}^{\pi}\right)}=-\frac{1}{\sqrt{2}} C$ & $A_{\Xi \Omega_{\mu}}^{\left(a_{\mu}^{K}\right)}=-C$ & \\
\hline
\end{tabular}

TABLE XXI. CGCs $A_{\bar{B} B}^{(i)}$ with axial-vector current $i$ and $\bar{B}, B \in[10]$, denoted by $\mu$.

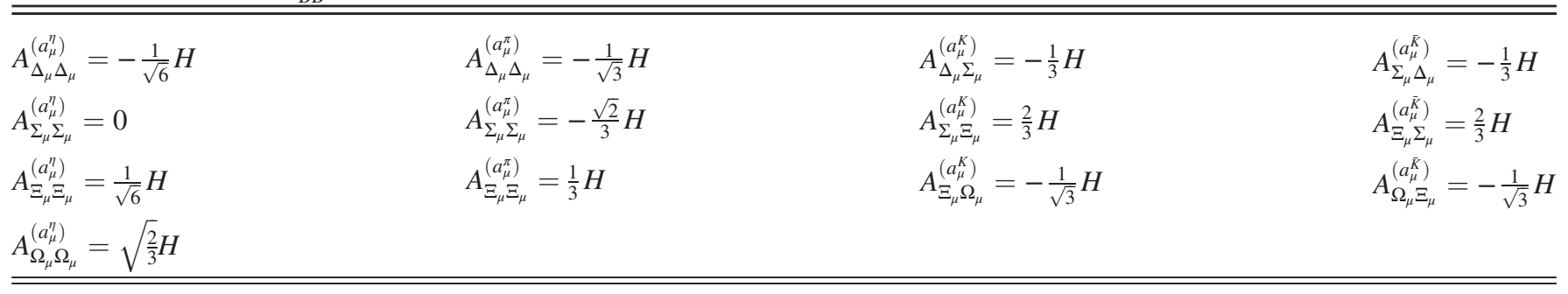

$$
\tilde{i}= \begin{cases}1 & \text { for } i=1 \\ 6 & \text { for } i=4, \\ 4 & \text { for } i=6 \\ 8 & \text { for } i=8\end{cases}
$$

\section{APPENDIX E: RECOUPLING CONSTANTS}

The recoupling constants of the bubble diagrams $\tilde{C}_{i}^{\bar{B} B}$ are shown in Table XXVIII.

The coefficients $\tilde{C}_{\bar{i}}^{B \bar{B}}$ in Eq. (31), connected to the bubble diagram with internal baryon $R$, are derived from $\tilde{C}_{i}^{\bar{B} B}$ by

TABLE XXII. The 5-point vertex CGCs $A_{Q, i}^{(\bar{B} B)}$ with the axialvector current $i$, the Goldstone boson $Q$, and the baryons $\bar{B}, B \in[8],[10]$.

\begin{tabular}{|c|c|c|}
\hline$A_{\pi, a_{\mu}^{n}}^{(\bar{B} B)}=0$ & $A_{\pi, a_{\mu}^{\pi}}^{(\bar{B} B)}=-4 A_{\bar{B} B}^{\left(a_{\mu}^{\pi}\right)}$ & $A_{\pi, a_{\mu}^{K}}^{(\bar{B} B)}=-\frac{3}{2} A_{\bar{B} B}^{\left(a_{\mu}^{K}\right)}$ \\
\hline$A_{K, a_{\mu}^{\eta}}^{(\bar{B} B)}=-3 A_{\bar{B} B}^{\left(a_{\mu}^{\eta}\right)}$ & $A_{K, a_{\mu}^{\pi}}^{(\bar{B} B)}=-A_{\bar{B} B}^{\left(a_{\mu}^{\pi}\right)}$ & $A_{K, a_{\mu}^{K}}^{(\bar{B} B)}=A_{\bar{K}, a_{\mu}^{K}}^{(\bar{B} B)}=0$ \\
\hline$A_{\bar{K}, a_{\mu}^{\eta}}^{(\bar{B} B)}=-3 A_{\bar{B} B}^{\left(a_{\mu}^{\eta}\right)}$ & $A_{\bar{K}, a_{\mu}^{\pi}}^{(\bar{B} B)}=-A_{\bar{B} B}^{\left(a_{\mu}^{\pi}\right)}$ & $A_{\bar{K}, a_{\mu}^{K}}^{(\bar{B} B)}=-3 A_{\bar{B} B}^{\left(a_{\mu}^{K}\right)}$ \\
\hline$A_{\eta, a_{\mu}^{\eta}}^{(\bar{B} B \bar{b}}=0$ & $A_{\eta, a_{\mu}^{\pi}}^{(\bar{B} B)}=0$ & $-\frac{3}{2} A_{\bar{B} B}^{\left(a_{\mu}^{K}\right)}$ \\
\hline$\left.A_{\pi, a_{\mu}^{\bar{K}}}^{(\bar{B} B \bar{B}}\right)=-\frac{3}{2} A_{\bar{B} B}^{\left(a_{\mu}^{\bar{K}}\right)}$ & $A_{K, a_{\mu}^{\bar{K}}}^{(\bar{B} B)}=-3 A_{\bar{B} B}^{\left(a_{\mu}^{\bar{K}}\right)}$ & $A_{\eta, a_{\mu}^{\bar{K}}}^{(\bar{B} B)}=-\frac{3}{2} A_{\bar{B} B}^{\left(a_{\mu}^{\bar{K}}\right)}$ \\
\hline
\end{tabular}

TABLE XXIII. CGCs $A_{Q L,(\bar{B})}^{(i B), 1}$ with the axial-vector-current index $i$, meson $Q$, and the baryons $\bar{B}, L, B \in[8]$. All CGCs satisfy $A_{Q L,(\bar{B})}^{(i B), 1}=A_{i B,(\bar{B})}^{(Q L), 1}$. Not all contributions are shown here.

\begin{tabular}{|c|c|c|}
\hline \multirow[b]{2}{*}{$A_{\pi N,(N)}^{\left(a_{\mu}^{\pi} N\right), 1}=-2 \sqrt{2}$} & & $A_{\pi \Lambda, \Sigma}^{\left(a_{\mu}^{\bar{K}} N\right), 1}=-\sqrt{3}$ \\
\hline & $\begin{array}{l}A_{\pi \Sigma,(\Lambda)}^{\left(a_{\mu}^{\pi} \Sigma\right), 1}=-4 \sqrt{2} \\
A_{\pi \Sigma,(\Lambda)}^{\left(a_{\mu}^{\bar{K}} N\right), 1}=-\sqrt{3}\end{array}$ & $\begin{array}{l}A_{\pi \Lambda,(\Sigma)}^{\left(a_{\mu}^{K} \Xi\right), 1}=-\sqrt{3} \\
A_{\pi \Sigma,(\Sigma)}^{\left(a_{\mu}^{\pi} \Sigma\right), 1}=-2 \sqrt{2}\end{array}$ \\
\hline$A_{\pi N,(N)}^{\left(a_{\mu}^{K} \Lambda\right), 1}=\frac{3}{\sqrt{2}}$ & $A_{\pi \Sigma,(\Lambda)}^{\left(a_{\mu}^{K} \Xi\right), 1}=\sqrt{3}$ & $A_{\pi \Sigma,(\Sigma)}^{\left(a_{\mu}^{\bar{K}} N\right), 1}=-\sqrt{2}$ \\
\hline$A_{\pi N,(N)}^{\left(a_{\mu}^{K} \Sigma\right), 1}=-\frac{1}{\sqrt{2}}$ & $A_{\bar{K} N,(\Lambda)}^{\left(a_{\mu}^{\vec{K}} N\right), 1}=-3 \sqrt{2}$ & $A_{\pi \Sigma,(\Sigma)}^{\left(a_{\mu}^{k} \Xi\right), 1}=\sqrt{2}$ \\
\hline$A_{\eta N,(N)}^{\left(a_{\mu}^{K} \Lambda\right), 1}=\frac{3}{\sqrt{2}}$ & $A_{\bar{K} N,(\Lambda)}^{\left(a_{\mu}^{n} \Lambda\right), 1}=-3$ & $A_{\bar{K} N,(\Sigma)}^{\left(a_{\mu}^{\bar{K}} N\right), 1}=-\sqrt{2}$ \\
\hline$A_{\eta N,(N)}^{\left(a_{\mu}^{K} \Sigma\right), 1}=\frac{3}{\sqrt{2}}$ & $A_{\eta \Lambda,(\Lambda)}^{\left(a_{\mu}^{K} \Xi\right), 1}=3$ & $A_{\bar{K} N,(\Sigma)}^{\left(a_{\mu}^{n} \Sigma\right), 1}=-\sqrt{3}$ \\
\hline$A_{K \Sigma,(N)}^{\left(a_{\mu}^{K} \Sigma\right), 1}=-2 \sqrt{2}$ & $A_{K \Xi,(\Lambda)}^{\left(a_{\mu}^{K} \Xi\right), 1}=-3 \sqrt{2}$ & $\begin{array}{l}A_{\eta \Sigma,(\Sigma)}^{\left(a_{\mu}^{K} \Xi\right), 1}=-\sqrt{3} \\
A_{K \Xi,(\Sigma)}^{\left(a_{\mu}^{K} \Xi\right), 1}=-\sqrt{2}\end{array}$ \\
\hline$A_{\pi \Xi,(\Xi)}^{\left(a_{\mu}^{\pi \Xi}\right), 1}=-2 \sqrt{2}$ & $A_{\pi \Xi,(\Xi)}^{\left(a_{\mu}^{\bar{K}} \Sigma\right), 1}=-\frac{1}{\sqrt{2}}$ & $A_{\bar{K} \Sigma,(\Xi)}^{\left(a_{\mu}^{\bar{K}} \Sigma\right), 1}=-2 \sqrt{2}$ \\
\hline$A_{\pi \Xi,(\Xi)}^{\left(a_{\mu}^{\bar{K}} \Lambda\right), 1}=-\frac{3}{\sqrt{2}}$ & $A_{\bar{K} \Lambda,(\Xi)}^{\left(a_{\mu}^{n} \Xi\right), 1}=\frac{3}{\sqrt{2}}$ & $A_{\bar{K} \Sigma,(\Xi)}^{\left(a_{\mu}^{n} \Xi\right), 1}=-\frac{3}{\sqrt{2}}$ \\
\hline
\end{tabular}


TABLE XXIV. CGCs $A_{Q L,(\bar{B})}^{(i B), 2}$ with the axial-vector-current index $i$, meson $Q$, and the baryons $\bar{B}, L, B \in[8]$. All CGCs satisfy $A_{Q L,(\bar{B})}^{(i B), 2}=A_{i B,(\bar{B})}^{(Q L), 2}$. Not all contributions are shown here.

\begin{tabular}{|c|c|}
\hline $\begin{array}{l}A_{\pi N,(N)}^{\left(a_{\mu}^{\pi} N\right), 2}=-\frac{1}{\sqrt{2}}\left(2 g_{0}^{(S)}+g_{D}^{(S)}+g_{F}^{(S)}\right) \\
A_{\pi N,(N)}^{\left(a_{\mu}^{\eta} N\right), 2}=-\frac{1}{\sqrt{2}}\left(g_{D}^{(S)}+g_{F}^{(S)}\right) \\
A_{\pi N,(N)}^{\left(a_{\mu}^{K} \Lambda\right), 2}=\frac{1}{2 \sqrt{2}}\left(g_{D}^{(S)}+3 g_{F}^{(S)}\right) \\
A_{\pi N,(N)}^{\left(a_{\mu}^{K} \Sigma\right), 2}=-\frac{1}{2 \sqrt{2}}\left(2 g_{1}^{(S)}-g_{D}^{(S)}+g_{F}^{(S)}\right) \\
A_{\eta N,(N)}^{\left(a_{\mu}^{\eta} N\right), 2}=\frac{-1}{3 \sqrt{2}}\left(6 g_{0}^{(S)}+5 g_{D}^{(S)}-3 g_{F}^{(S)}\right) \\
A_{\eta N,(N)}^{\left(a_{\mu}^{K} \Lambda\right), 2}=\frac{-1}{6 \sqrt{2}}\left(6 g_{1}^{(S)}+g_{D}^{(S)}+3 g_{F}^{(S)}\right) \\
A_{\eta N,(N)}^{\left(a_{\mu}^{K} \Sigma\right), 2}=\frac{1}{2 \sqrt{2}}\left(g_{D}^{(S)}-g_{F}^{(S)}\right) \\
A_{K \Lambda,(N)}^{\left(a_{\mu}^{K} \Lambda\right), 2}=-\sqrt{2} g_{0}^{(S)}-\frac{5}{3 \sqrt{2}} g_{D}^{(S)} \\
A_{K \Lambda,(N)}^{\left(a_{\mu}^{K} \Sigma\right), 2}=-\frac{1}{\sqrt{2}} g_{D}^{(S)} \\
A_{K \Sigma,(N)}^{\left(a_{\mu}^{K} \Sigma\right), 2}=-\frac{1}{\sqrt{2}}\left(2 g_{0}^{(S)}+g_{D}^{(S)}-2 g_{F}^{(S)}\right) \\
A_{\pi \Lambda,(\Sigma)}^{\left(a_{\mu}^{\pi} \Lambda\right), 2}=-\frac{\sqrt{2}}{3}\left(3 g_{0}^{(S)}+g_{D}^{(S)}\right) \\
A_{\pi \Lambda,(\Sigma)}^{\left(a_{\mu}^{K} N\right), 2}=\frac{1}{2 \sqrt{3}}\left(g_{D}^{(S)}+3 g_{F}^{(S)}\right) \\
A_{\pi \Lambda,(\Sigma)}^{\left(a_{\mu}^{\eta} \Sigma\right), 2}=-\frac{1}{\sqrt{2}} g_{1}^{(S)}-\frac{\sqrt{2}}{3} g_{D}^{(S)} \\
A_{\pi \Lambda,(\Sigma)}^{\left(a_{\mu}^{K} \Xi\right), 2}=\frac{1}{2 \sqrt{3}}\left(g_{D}^{(S)}-3 g_{F}^{(S)}\right) \\
A_{\pi \Sigma,(\Sigma)}^{\left(a_{\mu}^{\pi} \Sigma\right), 2}=\frac{-1}{\sqrt{2}}\left(2 g_{0}^{(S)}-g_{1}^{(S)}+2 g_{D}^{(S)}\right) \\
A_{\pi \Sigma,(\Sigma)}^{\left(a_{\mu}^{\bar{K}} N\right), 2}=\frac{1}{\sqrt{2}}\left(-g_{D}^{(S)}+g_{F}^{(S)}\right) \\
A_{\pi \Sigma,(\Sigma)}^{\left(a_{\mu}^{\eta} \Sigma\right), 2}=\frac{2}{\sqrt{3}} g_{F}^{(S)} \\
A_{\pi \Sigma,(\Sigma)}^{\left(a_{\mu}^{K} \Xi\right), 2}=\frac{1}{\sqrt{2}}\left(g_{D}^{(S)}+g_{F}^{(S)}\right) \\
A_{\bar{K} N,(\Sigma)}^{\left(a_{\mu}^{\bar{K}} N\right), 2}=\frac{-1}{\sqrt{2}}\left(2 g_{0}^{(S)}+g_{D}^{(S)}-g_{F}^{(S)}\right) \\
A_{\bar{K} N,(\Sigma)}^{\left(a_{\mu}^{\eta} \Sigma\right), 2}=\frac{1}{2 \sqrt{3}}\left(g_{D}^{(S)}-g_{F}^{(S)}\right) \\
A_{\bar{K} N,(\Sigma)}^{\left(a_{\mu}^{K} \Xi\right), 2}=-\frac{1}{\sqrt{2}} g_{1}^{(S)} \\
A_{\eta \Sigma,(\Sigma)}^{\left(a_{\mu}^{\eta} \Sigma\right), 2}=-\frac{\sqrt{2}}{3}\left(3 g_{0}^{(S)}+g_{D}^{(S)}\right) \\
A_{\eta \Sigma,(\Sigma)}^{\left(a_{\mu}^{K} \Xi\right), 2}=\frac{1}{2 \sqrt{3}}\left(g_{D}^{(S)}+g_{F}^{(S)}\right) \\
A_{K \Xi,(\Sigma)}^{\left(a_{\mu}^{K} \Xi\right), 2}=\frac{-1}{\sqrt{2}}\left(2 g_{0}^{(S)}+g_{D}^{(S)}+g_{F}^{(S)}\right) \\
\end{array}$ & $\begin{array}{l}A_{\pi \Sigma,(\Lambda)}^{\left(a_{\mu}^{\pi} \Sigma\right), 2}=-\sqrt{2}\left(g_{0}^{(S)}+2 g_{1}^{(S)}+g_{D}^{(S)}\right) \\
A_{\pi \Sigma,(\Lambda)}^{\left(a_{\mu}^{\bar{K}} N\right), 2}=\frac{\sqrt{3}}{2}\left(-2 g_{1}^{(S)}-g_{D}^{(S)}+g_{F}^{(S)}\right) \\
A_{\pi \Sigma,(\Lambda)}^{\left(a_{\mu}^{\eta} \Lambda\right), 2}=-\frac{1}{\sqrt{6}}\left(3 g_{1}^{(S)}+2 g_{D}^{(S)}\right) \\
A_{\pi \Sigma,(\Lambda)}^{\left(a_{\mu}^{K} \Xi\right), 2}=\frac{\sqrt{3}}{2}\left(2 g_{1}^{(S)}+g_{D}^{(S)}+g_{F}^{(S)}\right) \\
A_{\bar{K} N,(\Lambda)}^{\left(a_{\mu}^{\bar{K}} N\right), 2}=\frac{-1}{\sqrt{2}}\left(2 g_{0}^{(S)}+2 g_{1}^{(S)}+3 g_{D}^{(S)}+g_{F}^{(S)}\right) \\
A_{\bar{K} N,(\Lambda), 2}^{\left(a_{\mu}^{\eta} \Lambda\right),}=-\frac{1}{6}\left(6 g_{1}^{(S)}+g_{D}^{(S)}+3 g_{F}^{(S)}\right) \\
A_{\bar{K} N,(\Lambda)}^{\left(a_{\mu}^{K} \Xi\right), 2}=\frac{3}{\sqrt{2}} g_{1}^{(S)} \\
A_{\eta \Lambda,(\Lambda)}^{\left(a_{\mu}^{\eta} \Lambda\right), 2}=-\sqrt{2}\left(g_{0}^{(S)}+g_{1}^{(S)}+g_{D}^{(S)}\right) \\
A_{\eta \Lambda,(\Lambda)}^{\left(a_{\mu}^{K} \Xi\right), 2}=\frac{1}{6}\left(6 g_{1}^{(S)}+g_{D}^{(S)}-3 g_{F}^{(S)}\right) \\
A_{K \Xi,(\Lambda)}^{\left(a_{\mu}^{K} \Xi\right), 2}=\frac{-1}{\sqrt{2}}\left(2 g_{0}^{(S)}+2 g_{1}^{(S)}+3 g_{D}^{(S)}-g_{F}^{(S)}\right)\end{array}$ \\
\hline
\end{tabular}

switching the incoming and outgoing external baryon. Note that the axial-vector current also needs to be turned around $[i \leftrightarrow \bar{i}$; see Eq. (D4)]. In the isospin basis this only affects the strangeness-changing current, implying $a_{\mu}^{K} \leftrightarrow a_{\mu}^{\bar{K}}$.

For the recoupling constants of the triangle diagrams we introduce a new notation for internal particles with the same isospin and strangeness: $\Xi_{*}$ includes the octet $\Xi$ and the decuplet $\Xi_{\mu}$, and $\Sigma_{*}$ includes the octet $\Sigma$ and the decuplet $\Sigma_{\mu}$. We find a simple structure for the recoupling constants with axial-vector current $a_{\mu}^{\eta}$ :

$$
\tilde{C}_{\bar{B} B, L R}^{a_{\mu}^{\eta} Q}=1
$$

for all $Q, \bar{B}, B, L$, and $R$.
The isospin structure of all other axial-vector currents complicates the treatment. We give all recoupling constants explicitly in Tables XXIX and XXX.

The recoupling constants with axial-vector current $a_{\mu}^{\bar{K}}$ are derived from the recoupling constant $\tilde{C}_{\bar{B} B, L R}^{a_{\mu}^{K} Q}$ by

$$
\tilde{C}_{\bar{B} B, L R}^{a_{\mu}^{\bar{K}} Q}=\tilde{C}_{B \bar{B}, R L}^{a_{\mu}^{K} Q}
$$

\section{APPENDIX F: KINEMATIC FACTORS}

Prefactors $K_{a b c}^{(A B C)}$ in Eq. (33) are connected to the integral involving the internal particles $A, B, C$ and originating from a diagram with internal particles $a, b, c$. 
$\begin{array}{llllll}\text { TABLE } & X X V \\ \text { shown here. }\end{array}$ shown here.

\begin{tabular}{|c|c|}
\hline 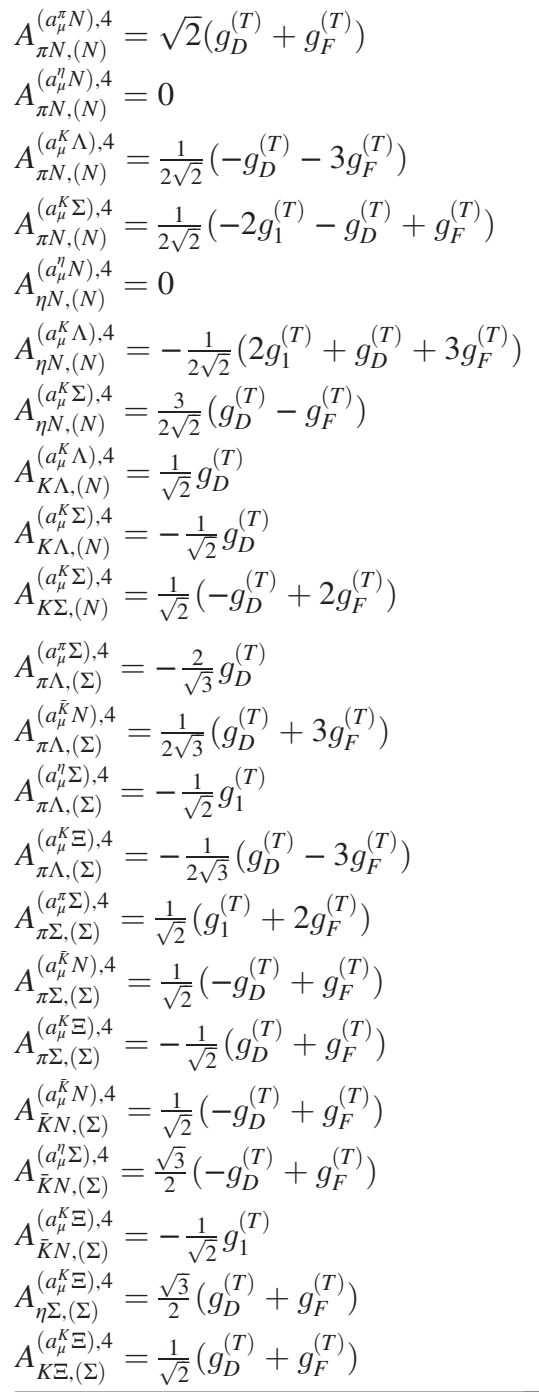 & $\begin{array}{l}A_{\pi \Sigma,(\Lambda)}^{\left(a_{\mu}^{\pi} \Sigma\right), 4}=\sqrt{2}\left(g_{1}^{(T)}+2 g_{F}^{(T)}\right) \\
A_{\pi \Sigma,(\Lambda)}^{\left(a_{\mu}^{K} N\right), 4}=\frac{\sqrt{3}}{2}\left(2 g_{1}^{(T)}-g_{D}^{(T)}+g_{F}^{(T)}\right) \\
A_{\pi \Sigma,(\Lambda)}^{\left(a_{\mu}^{\eta} \Lambda\right), 4}=\sqrt{\frac{3}{2}} g_{1}^{(T)} \\
A_{\pi \Sigma,(\Lambda)}^{\left(a_{\mu}^{K} \Xi\right), 4}=-\frac{\sqrt{3}}{2}\left(2 g_{1}^{(T)}+g_{D}^{(T)}+g_{F}^{(T)}\right) \\
A_{\bar{K} N,(\Lambda)}^{\left(a_{\bar{K}}^{\bar{K}} N\right), 4}=\frac{1}{\sqrt{2}}\left(2 g_{1}^{(T)}+g_{D}^{(T)}+3 g_{F}^{(T)}\right) \\
A_{\bar{K} N,(\Lambda)}^{\left(a_{\mu}^{\eta} \Lambda\right), 4}=\frac{1}{2}\left(2 g_{1}^{(T)}+g_{D}^{(T)}+3 g_{F}^{(T)}\right) \\
A_{\bar{K} N,(\Lambda)}^{\left(a_{\mu}^{K} \Xi\right), 4}=-\frac{1}{\sqrt{2}} g_{1}^{(T)} \\
A_{\eta \Lambda,(\Lambda)}^{\left(a_{\mu}^{\eta} \Lambda\right), 4}=0 \\
A_{\eta \Lambda,(\Lambda)}^{\left(a_{\mu}^{K} \Xi\right), 4}=\frac{1}{2}\left(-2 g_{1}^{(T)}+g_{D}^{(T)}-3 g_{F}^{(T)}\right) \\
A_{K \Xi,(\Lambda)}^{\left(a_{\mu}^{K} \Xi\right), 4}=\frac{1}{\sqrt{2}}\left(2 g_{1}^{(T)}-g_{D}^{(T)}+3 g_{F}^{(T)}\right)\end{array}$ \\
\hline
\end{tabular}

TABLE XXVI. CGCs $A_{Q L,(\bar{B})}^{(i B), 1}$ with $L \in[10]$.

\begin{tabular}{ll}
\hline \hline$A_{\pi \Delta_{\mu},(N)}^{\left(a_{\mu}^{\pi} N\right), 1}=\frac{5}{2 \sqrt{6}} f_{3}^{(A)}$ & $A_{\pi \Sigma_{\mu},(\Lambda)}^{\left(a_{\mu}^{\pi} \Sigma\right), 1}=-\frac{1}{2 \sqrt{6}}\left(f_{1}^{(A)}+3 f_{3}^{(A)}\right)$ \\
$A_{\pi \Delta_{\mu},(N)}^{\left(a_{\mu}^{\eta} N\right), 1}=\frac{1}{2 \sqrt{6}}\left(-2 f_{1}^{(A)}+f_{3}^{(A)}\right)$ & $A_{\pi \Sigma_{\mu},(\Lambda)}^{\left(a_{\mu}^{K} N\right), 1}=-\frac{1}{4}\left(f_{1}^{(A)}-3 f_{3}^{(A)}\right)$ \\
$A_{\pi \Delta_{\mu},(N)}^{\left(a_{\mu}^{K} \Lambda\right), 1}=-\frac{\sqrt{3}}{2 \sqrt{2}} f_{3}^{(A)}$ & $A_{\pi \Sigma_{\mu},(\Lambda)}^{\left(a_{\mu}^{\eta} \Lambda\right), 1}=\frac{1}{2 \sqrt{2}}\left(f_{1}^{(A)}+f_{3}^{(A)}\right)$ \\
$A_{\pi \Delta_{\mu},(N)}^{\left(a_{\mu}^{K} \Sigma\right), 1}=\frac{1}{2 \sqrt{6}}\left(2 f_{1}^{(A)}+f_{3}^{(A)}\right)$ & $A_{\pi \Sigma_{\mu},(\Lambda)}^{\left(a_{\mu}^{K} \Xi\right), 1}=\frac{1}{4}\left(-f_{1}^{(A)}+f_{3}^{(A)}\right)$ \\
$A_{K \Sigma_{\mu},(N)}^{\left(a_{\mu}^{\pi} N\right), 1}=\frac{1}{4 \sqrt{6}}\left(f_{1}^{(A)}+5 f_{3}^{(A)}\right)$ & $A_{K \Xi_{\mu},(\Lambda)}^{\left(a_{\mu}^{\pi} \Sigma\right), 1}=-\frac{1}{4}\left(f_{1}^{(A)}+2 f_{3}^{(A)}\right)$ \\
$A_{K \Sigma_{\mu},(N)}^{\left(a_{\mu}^{\eta} N\right), 1}=\frac{1}{4 \sqrt{6}}\left(f_{1}^{(A)}+f_{3}^{(A)}\right)$ & $A_{K \Xi_{\mu},(\Lambda)}^{\left(a_{\mu}^{K} N\right), 1}=\frac{\sqrt{3}}{2 \sqrt{2}} f_{3}^{(A)}$ \\
$A_{K \Sigma_{\mu},(N)}^{\left(a_{\mu}^{K} \Lambda\right), 1}=\frac{1}{4 \sqrt{6}}\left(3 f_{1}^{(A)}-3 f_{3}^{(A)}\right)$ & $A_{K \Xi_{\mu},(\Lambda)}^{\left(a_{\mu}^{\eta} \Lambda\right), 1}=-\frac{1}{4 \sqrt{3}}\left(f_{1}^{(A)}-2 f_{3}^{(A)}\right)$ \\
$A_{K \Sigma_{\mu},(N)}^{\left(a_{\mu}^{K} \Sigma\right), 1}=\frac{1}{4 \sqrt{6}}\left(3 f_{1}^{(A)}+f_{3}^{(A)}\right)$ & $A_{K \Xi_{\mu},(\Lambda)}^{\left(a_{\mu}^{K} \Xi\right), 1}=\frac{1}{2 \sqrt{6}}\left(-2 f_{1}^{(A)}+f_{3}^{(A)}\right)$ \\
$A_{\pi \Sigma_{\mu},(\Sigma)}^{\left(a_{\mu}^{\pi} \Sigma\right), 1}=\frac{1}{2 \sqrt{6}}\left(-f_{1}^{(A)}+2 f_{3}^{(A)}\right)$ & $A_{\pi \Xi_{\mu},(\Xi)}^{\left(a_{\mu}^{\pi} \Xi\right), 1}=\frac{1}{2 \sqrt{6}} f_{1}^{(A)}$ \\
\hline
\end{tabular}

(Table continued)
TABLE XXVI. (Continued)

\begin{tabular}{ll}
\hline \hline$A_{\pi \Sigma_{\mu},(\Sigma)}^{\left(a_{\bar{K}}^{\bar{K}} N\right), 1}=-\frac{1}{2 \sqrt{6}}\left(f_{1}^{(A)}+f_{3}^{(A)}\right)$ & $A_{\pi \Xi_{\mu},(\Xi)}^{\left(a_{\mu}^{\bar{K}} \Lambda\right), 1}=\frac{\sqrt{3}}{4 \sqrt{2}} f_{1}^{(A)}$ \\
$A_{\pi \Sigma_{\mu},(\Sigma)}^{\left(a_{\mu}^{\eta} \Sigma\right), 1}=\frac{1}{6}\left(f_{1}^{(A)}-2 f_{3}^{(A)}\right)$ & $A_{\pi \Xi_{\mu},(\Xi)}^{\left(a_{\mu}^{\bar{K}} \Sigma\right), 1}=-\frac{1}{4 \sqrt{6}}\left(f_{1}^{(A)}-6 f_{3}^{(A)}\right)$ \\
$A_{\pi \Sigma_{\mu},(\Sigma)}^{\left(a_{\mu}^{K} \Xi\right), 1}=-\frac{1}{2 \sqrt{6}}\left(f_{1}^{(A)}+f_{3}^{(A)}\right)$ & $A_{\pi \Xi_{\mu},(\Xi)}^{\left(a_{\mu}^{\eta} \Xi\right), 1}=\frac{1}{2 \sqrt{6}}\left(f_{1}^{(A)}+f_{3}^{(A)}\right)$ \\
$A_{\bar{K} \Delta_{\mu},(\Sigma)}^{\left(a_{\mu}^{\pi} \Lambda\right), 1}=-\frac{1}{2} f_{3}^{(A)}$ & $A_{\bar{K} \Sigma_{\mu},(\Xi)}^{\left(a_{\mu}^{\pi} \Xi\right), 1}=-\frac{1}{4 \sqrt{6}}\left(f_{1}^{(A)}+3 f_{3}^{(A)}\right)$ \\
$A_{\bar{K} \Delta_{\mu},(\Sigma)}^{\left(a_{\mu}^{\pi} \Sigma\right), 1}=-\frac{1}{2 \sqrt{6}}\left(f_{1}^{(A)}-2 f_{3}^{(A)}\right)$ & $A_{\bar{K} \Sigma_{\mu},(\Xi)}^{\left(a_{\mu}^{K} \Lambda\right), 1}=-\frac{\sqrt{3}}{4 \sqrt{2}}\left(f_{1}^{(A)}-f_{3}^{(A)}\right)$ \\
$A_{\bar{K} \Delta_{\mu},(\Sigma)}^{\left(a_{\bar{K}}^{\bar{K}}\right), 1}=-\frac{1}{\sqrt{6}} f_{1}^{(A)}$ & $A_{\bar{K} \Sigma_{\mu},(\Xi)}^{\left(a_{\bar{K}}^{\bar{K}} \Sigma\right), 1}=-\frac{1}{4 \sqrt{6}}\left(f_{1}^{(A)}+3 f_{3}^{(A)}\right)$ \\
$A_{\bar{K} \Delta_{\mu},(\Sigma)}^{\left(a_{\mu}^{\eta} \Sigma\right), 1}=-\frac{1}{6}\left(f_{1}^{(A)}+f_{3}^{(A)}\right)$ & $A_{\bar{K} \Sigma_{\mu},(\Xi)}^{\left(a_{\mu}^{\eta} \Xi\right), 1}=\frac{1}{4 \sqrt{6}}\left(f_{1}^{(A)}-5 f_{3}^{(A)}\right)$ \\
$A_{\bar{K} \Delta_{\mu},(\Sigma)}^{\left(a_{K}^{K} \Xi\right), 1}=-\frac{\sqrt{2}}{\sqrt{3}} f_{3}^{(A)}$ & $A_{\eta \Xi_{\mu},(\Xi)}^{\left(a_{\mu}^{\pi} \Xi\right), 1}=\frac{1}{2 \sqrt{6}}\left(f_{1}^{(A)}+f_{3}^{(A)}\right)$ \\
$A_{\eta \Sigma_{\mu},(\Sigma)}^{\left(a_{\mu}^{\pi} \Lambda\right), 1}=\frac{1}{2 \sqrt{6}}\left(f_{1}^{(A)}+f_{3}^{(A)}\right)$ & $A_{\eta \Xi_{\mu},(\Xi)}^{\left(a_{\mu}^{K} \Lambda\right), 1}=\frac{1}{4 \sqrt{6}}\left(f_{1}^{(A)}-2 f_{3}^{(A)}\right)$ \\
\hline
\end{tabular}

(Table continued) 
TABLE XXVI. (Continued)

\begin{tabular}{|c|c|}
\hline $\begin{array}{l}A_{\eta \Sigma_{\mu},(\Sigma)}^{\left(a_{\pi}^{\pi} \Sigma\right), 1}=\frac{1}{6}\left(f_{1}^{(A)}-2 f_{3}^{(A)}\right) \\
A_{\eta \Sigma_{\mu},(\Sigma)}^{\left(a_{\bar{K}}^{\bar{K}} N\right), 1}=\frac{1}{12}\left(f_{1}^{(A)}+f_{3}^{(A)}\right) \\
A_{\eta \Sigma_{\mu},(\Sigma)}^{\left(a_{\mu}^{\eta} \Sigma\right), 1}=\frac{1}{2 \sqrt{6}}\left(f_{1}^{(A)}+f_{3}^{(A)}\right) \\
A_{\eta \Sigma_{\mu},(\Sigma)}^{\left(a_{\mu}^{K} \Xi\right),}=\frac{1}{12}\left(-f_{1}^{(A)}+5 f_{3}^{(A)}\right) \\
A_{K \Xi_{\mu},(\Sigma)}^{\left(a_{\mu}^{\pi} \Lambda\right), 1}=-\frac{1}{4} f_{1}^{(A)} \\
A_{K \Xi_{\mu},(\Sigma)}^{\left(a_{\mu}^{\pi} \Sigma\right), 1}=\frac{1}{2 \sqrt{6}}\left(-f_{1}^{(A)}+2 f_{3}^{(A)}\right) \\
A_{K \Xi_{\mu},(\Sigma)}^{\left(a_{\mu}^{K} N\right), 1}=-\frac{1}{2 \sqrt{6}} f_{3}^{(A)}\end{array}$ & $\begin{aligned} A_{\eta \Xi_{\mu},(\Xi)}^{\left(a_{\mu}^{\bar{K}} \Sigma\right), 1} & =\frac{1}{4 \sqrt{6}}\left(f_{1}^{(A)}+4 f_{3}^{(A)}\right) \\
A_{\eta \Xi_{\mu},(\Xi)}^{\left(a_{\mu}^{\eta} \Xi\right), 1} & =-\frac{1}{2 \sqrt{6}}\left(f_{1}^{(A)}-2 f_{3}^{(A)}\right) \\
A_{K \Omega_{\mu},(\Xi)}^{\left(a_{\mu}^{\pi} \Xi\right), 1} & =\frac{\sqrt{3}}{4} f_{1}^{(A)} \\
A_{K \Omega_{\mu},(\Xi)}^{\left(a_{\mu}^{\bar{K}} \Sigma\right), 1} & =\frac{\sqrt{3}}{2} f_{3}^{(A)} \\
A_{K \Omega_{\mu},(\Xi)}^{\left(a_{\mu}^{\eta} \Xi\right), 1} & =-\frac{1}{4 \sqrt{3}}\left(f_{1}^{(A)}-2 f_{3}^{(A)}\right) \\
A_{K \Xi_{\mu},(\Sigma)}^{\left(a_{\mu}^{\eta} \Sigma\right), 1} & =-\frac{1}{12}\left(f_{1}^{(A)}+4 f_{3}^{(A)}\right) \\
A_{K \Xi_{\mu},(\Sigma)}^{\left(a_{\mu}^{K} \Xi\right), 1} & =-\frac{1}{2 \sqrt{6}} f_{3}^{(A)}\end{aligned}$ \\
\hline
\end{tabular}

TABLE XXVII. CGCs $A_{Q L,(\bar{B})}^{(i B), 2}$ with $L \in[10]$.

\begin{tabular}{|c|c|}
\hline 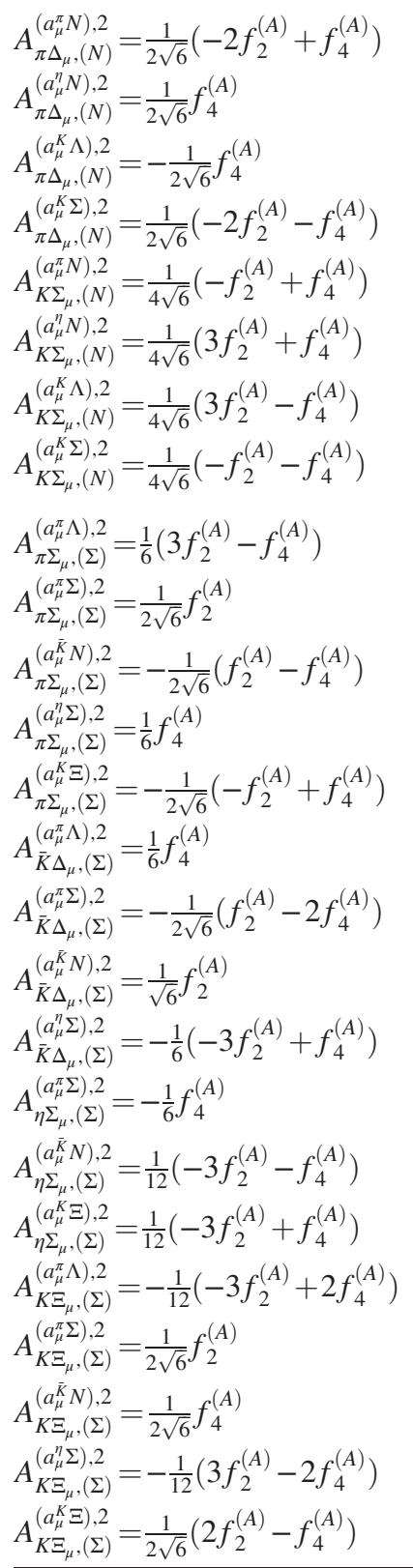 & 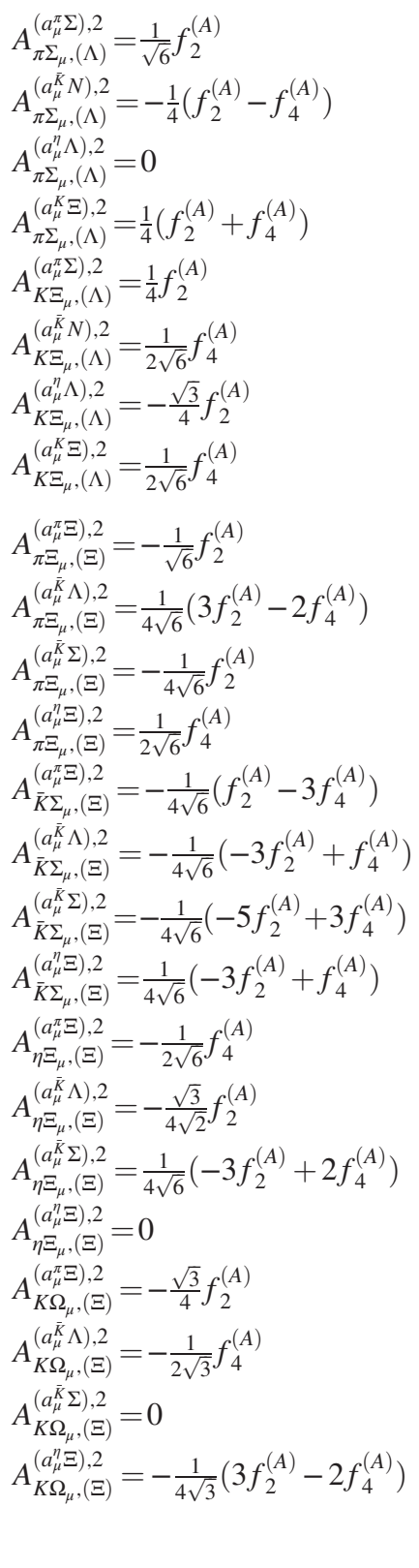 \\
\hline
\end{tabular}

TABLE XXVIII. Recoupling constants $\tilde{C}_{i}^{\bar{B} B}$ of the bubble diagram with internal baryon $L$.

\begin{tabular}{llll}
\hline \hline$\tilde{C}_{a_{\mu}^{\pi}}^{N N}=-\sqrt{\frac{2}{3}}$ & $\tilde{C}_{a_{\mu}^{K}}^{\Sigma \Xi}=-1$ & $\tilde{C}_{a_{\mu}^{\bar{K}}}^{\Xi \Sigma}=\sqrt{\frac{2}{3}}$ & $\tilde{C}_{a_{\mu}^{\eta}}^{N N}=-1$ \\
$\tilde{C}_{a_{\mu}^{\pi}}^{\Sigma \Sigma}=\frac{1}{\sqrt{2}}$ & $\tilde{C}_{a_{\mu}^{K}}^{N \Sigma}=-\sqrt{\frac{2}{3}}$ & $\tilde{C}_{a_{\mu}^{\bar{K}}}^{\Sigma N}=-1$ & $\tilde{C}_{a_{\mu}^{\eta}}^{\Sigma \Sigma}=-1$ \\
$\tilde{C}_{a_{\mu}^{\bar{T}}}^{\Xi \Xi}=\sqrt{\frac{2}{3}}$ & $\tilde{C}_{a_{\mu}^{K}}^{N \Lambda}=-1$ & $\tilde{C}_{a_{\mu}^{\bar{K}}}^{\Lambda N}=-\frac{1}{\sqrt{2}}$ & $\tilde{C}_{a_{\mu}^{\eta}}^{\Xi \Xi}=-1$ \\
$\tilde{C}_{a_{\mu}^{\pi}}^{\Lambda \Sigma}=-\frac{1}{\sqrt{3}}$ & $\tilde{C}_{a_{\mu}^{K}}^{\Lambda \Xi}=\frac{1}{\sqrt{2}}$ & $\tilde{C}_{a_{\mu}^{\bar{K}}}^{\Xi \Lambda}=-1$ & $\tilde{C}_{a_{\mu}^{\eta}}^{\Lambda \Lambda}=-1$ \\
$\tilde{C}_{a_{\mu}^{\pi}}^{\Sigma \Lambda}=-1$ & & & \\
\hline \hline
\end{tabular}

Power-counting violating terms are subtracted: $K_{a b c}^{(A B C)} \rightarrow$ $\bar{K}_{a b c}^{(A B C)}$. We expand $\bar{K}_{a b c}^{(A B C)}$ in terms of the parameters $m_{Q}^{2}$, $t, \delta_{L}, \delta_{R}$, and $\delta_{B}(40)$ in this appendix:

$$
\begin{aligned}
\bar{K}_{Q}^{(Q)} & =1, \\
\bar{K}_{L \in[8] Q}^{(Q), n \in\{1,2,3,4,5\}} & =\bar{K}_{Q R \in[8]}^{(Q), n \in\{1,2,3,4,5\}}=O\left(Q^{2}\right), \\
\bar{K}_{L \in[8] Q R \in[8]}^{(Q), n=1} & =-1+O\left(Q^{2}\right), \\
\bar{K}_{L \in[10] Q R \in[8]}^{(Q), n \in\{1,2\}} & =\bar{K}_{L \in[8] Q R \in[10]}^{(Q), n \in\{1,2\}}=O\left(Q^{2}\right), \\
\bar{K}_{L \in[10] Q R \in[10]}^{(Q), 1} & =O\left(Q^{2}\right), \\
\bar{K}_{L \in[10] Q}^{(Q), n \in\{1,2,3\}} & =\bar{K}_{Q R \in[10]}^{(Q), n \in\{1,2,3\}}=O\left(Q^{2}\right),
\end{aligned}
$$

TABLE XXIX. Recoupling constants with axial-vector current $a_{\mu}^{\pi}: \tilde{C}_{\bar{B} B, L R}^{a_{\mu}^{\pi} Q}$.

\begin{tabular}{lll}
\hline \hline$\tilde{C}_{\Sigma \Sigma, \Sigma_{*} \Sigma_{*}}^{a_{\mu}^{\pi} \pi}=\frac{1}{2}$ & $\tilde{C}_{N N, \Sigma_{*} \Sigma_{*}}^{a_{\mu}^{\pi} K}=\frac{2 \sqrt{2}}{3}$ & $\tilde{C}_{\Xi \Xi, \Sigma_{*} \Sigma_{*}^{\pi} \overline{a_{*}}}^{a_{\mu}}=-\frac{2 \sqrt{2}}{3}$ \\
$\tilde{C}_{\Sigma \Sigma, N N}^{a_{\mu}^{\pi} \bar{K}}=\frac{1}{\sqrt{2}}$ & $\tilde{C}_{N N, N N}^{a_{\mu}^{\pi} \pi}=-\frac{1}{3}$ & $\tilde{C}_{\Xi \Xi, \Xi_{*} \Xi_{*}}^{a_{\mu}^{\pi} \pi}=-\frac{1}{3}$ \\
$\tilde{C}_{\Sigma \Sigma, N \Delta}^{a_{\mu}^{\pi} \bar{K}}=-\frac{1}{\sqrt{6}}$ & $\tilde{C}_{N N, N N}^{a_{\mu}^{\pi} \eta}=1$ & $\tilde{C}_{\Xi \Xi, \Xi_{*} \Xi_{*}}^{a_{\mu}^{\pi} \eta}=1$ \\
$\tilde{C}_{\Sigma \Sigma, \Delta N}^{a_{\mu}^{\pi} \bar{K}}=-\frac{1}{\sqrt{6}}$ & $\tilde{C}_{N N, N \Delta}^{a_{\mu}^{\pi} \pi}=\frac{4}{3 \sqrt{3}}$ & $\tilde{C}_{\Xi \Xi, \Sigma_{*} \Lambda}^{a_{\mu}^{\pi} \bar{K}}=-\frac{\sqrt{2}}{\sqrt{3}}$ \\
$\tilde{C}_{\Sigma \Sigma, \Xi_{*} \Xi_{*}}^{a_{\mu}^{\pi} K}=-\frac{1}{\sqrt{2}}$ & $\tilde{C}_{N N, \Delta N}^{a_{\mu}^{\pi} \pi}=\frac{4}{3 \sqrt{3}}$ & $\tilde{C}_{\Xi \Xi, \Lambda \Sigma_{*}}^{a_{\mu}^{\pi} \bar{K}}=-\frac{\sqrt{2}}{\sqrt{3}}$ \\
$\tilde{C}_{\Sigma \Sigma, \Delta \Delta}^{a_{\mu}^{\pi} \bar{K}}=\frac{5}{2 \sqrt{6}}$ & $\tilde{C}_{N N, \Delta \Delta}^{a_{\mu}^{\pi} \pi}=\frac{5}{3 \sqrt{3}}$ & \\
$\tilde{C}_{\Sigma \Sigma, \Sigma_{*} \Sigma_{*}}^{a_{\mu}^{\pi} \eta}=1$ & $\tilde{C}_{N N, \Sigma_{*} \Lambda}^{a_{\mu}^{\pi} K}=\frac{\sqrt{2}}{\sqrt{3}}$ & \\
$\tilde{C}_{\Sigma \Sigma, \Lambda \Sigma_{*}}^{a_{\mu}^{\pi} \pi}=\frac{1}{\sqrt{2}}$ & $\tilde{C}_{N N, \Lambda \Sigma_{*}}^{a_{\mu}^{\pi} K}=\frac{\sqrt{2}}{\sqrt{3}}$ & \\
$\tilde{C}_{\Sigma \Sigma, \Sigma_{*} \Lambda}^{a_{\mu}^{\pi} \pi}=\frac{1}{\sqrt{2}}$ & \\
$\tilde{C}_{\Sigma \Lambda, \Sigma_{*} \Sigma_{*}}^{a_{\mu}^{\pi} \pi}=\sqrt{\frac{2}{3}}$ & $\tilde{C}_{\Lambda \Sigma, \Sigma_{*} \Sigma_{*}}^{a_{\mu}^{\pi} \pi}=\sqrt{\frac{2}{3}}$ & \\
$\tilde{C}_{\Sigma \Lambda, N N}^{a_{\mu}^{\pi} \bar{K}}=\frac{1}{\sqrt{2}}$ & $\tilde{C}_{\Lambda \Sigma, N N}^{a_{\mu}^{\pi} \bar{K}}=\frac{1}{\sqrt{2}}$ & \\
$\tilde{C}_{\Sigma \Lambda, \Xi_{*} \Xi_{*}}^{a_{\mu}^{\pi} K}=-\frac{1}{\sqrt{2}}$ & $\tilde{C}_{\Lambda \Sigma, \Xi_{*} \Xi_{*}}^{a_{\mu}^{\pi} K}=-\frac{1}{\sqrt{2}}$ & \\
$\tilde{C}_{\Sigma \Lambda, \Delta N}^{a_{\mu}^{\pi} \bar{K}}=\sqrt{\frac{2}{3}}$ & $\tilde{C}_{\Lambda \Sigma, N \Delta}^{a_{\mu}^{\pi} \bar{K}}=\sqrt{\frac{2}{3}}$ & \\
$\tilde{C}_{\Sigma \Lambda, \Lambda \Sigma_{*}}^{a_{\mu}^{\pi} \pi}=\frac{1}{\sqrt{3}}$ & $\tilde{C}_{\Lambda \Sigma, \Sigma_{*} \Lambda}^{a_{\mu}^{\pi} \pi}=\frac{1}{\sqrt{3}}$ & \\
$\tilde{C}_{\Sigma \Lambda, \Sigma_{*} \Lambda}^{a_{\mu}^{\pi} \eta}=1$ & $\tilde{C}_{\Lambda \Sigma, \Lambda \Sigma_{*}}^{a_{\mu}^{\pi} \eta}=1$ & \\
\hline \hline & & \\
\hline
\end{tabular}


TABLE XXX. Recoupling constants with axial-vector current $a_{\mu}^{K}: \tilde{C}_{\bar{B} B, L R}^{a_{\mu}^{K} Q}$.

\begin{tabular}{|c|c|c|c|}
\hline $\begin{array}{l}\tilde{C}_{\Sigma \Xi, \Sigma_{*} \Xi_{*}}^{a_{\mu}^{K} \pi}=-\frac{\sqrt{2}}{\sqrt{3}} \\
\tilde{C}_{\Sigma \Xi, \Sigma_{*} \Xi_{*}}^{a_{\mu}^{K} \eta}=1 \\
\tilde{C}_{\Sigma \Xi, N \Sigma_{*}}^{a_{\mu}^{K} \bar{K}}=\frac{1}{\sqrt{6}} \\
\tilde{C}_{\Sigma \Xi, N \Lambda}^{a_{\mu}^{K} \bar{K}}=1 \\
\tilde{C}_{\Sigma \Xi, \Xi_{*} \Omega}^{a_{\mu}^{K} K}=1 \\
\tilde{C}_{\Sigma \Xi, \Lambda \Xi}^{a_{\mu}^{K} \pi}=-\sqrt{\frac{2}{3}} \\
\tilde{C}_{\Sigma \Xi, \Delta \Sigma_{*}}^{a_{\mu}^{K} \bar{K}}=-2 \sqrt{\frac{2}{3}}\end{array}$ & $\begin{array}{l}\tilde{C}_{N \Sigma, \Sigma_{*} \Xi_{*}}^{a_{\mu}^{K} K}=-\frac{1}{\sqrt{6}} \\
\tilde{C}_{N \Sigma, N \Sigma_{*}}^{a_{\mu}^{K} \pi}=-\frac{\sqrt{2}}{\sqrt{3}} \\
\tilde{C}_{N \Sigma, N \Sigma_{*}}^{a_{\mu}^{K} \eta}=1 \\
\tilde{C}_{N \Sigma, N \Lambda}^{a_{\mu}^{K} \pi}=\sqrt{\frac{2}{3}} \\
\tilde{C}_{N \Sigma, \Lambda \Xi_{*}}^{a_{\mu}^{K} K}=1 \\
\tilde{C}_{N \Sigma, \Delta \Sigma_{*}}^{a_{\mu}^{K} \pi}=\sqrt{\frac{2}{3}}\end{array}$ & $\begin{array}{l}\tilde{C}_{N \Lambda, \Sigma_{*} \Xi_{*}}^{a_{\mu}^{K} K}=-\frac{\sqrt{3}}{2} \\
\tilde{C}_{N \Lambda, N \Sigma_{*}}^{a_{\mu}^{K} \pi}=\frac{1}{\sqrt{2}} \\
\tilde{C}_{N \Lambda, N \Lambda}^{a_{\mu}^{K} \eta}=1 \\
\tilde{C}_{N \Lambda, \Lambda \Xi_{*}}^{a_{\mu}^{K} K}=-\frac{1}{\sqrt{2}} \\
\tilde{C}_{N \Lambda, \Delta \Sigma_{*}}^{a_{\mu}^{K} \pi}=\sqrt{2}\end{array}$ & $\begin{array}{l}\tilde{C}_{\Lambda \Xi, \Sigma_{*} \Xi_{*}}^{a_{\mu}^{K} \pi}=-\frac{1}{\sqrt{2}} \\
\tilde{C}_{\Lambda \Xi, N \Sigma_{*}}^{a_{\mu}^{K} \bar{K}}=-\frac{\sqrt{3}}{2} \\
\tilde{C}_{\Lambda \Xi, N \Lambda}^{a_{\mu}^{K} \bar{K}}=\frac{1}{\sqrt{2}} \\
\tilde{C}_{\Lambda \Xi, \Xi_{*} \Omega}^{a_{\mu}^{K} K}=\frac{1}{\sqrt{2}} \\
\tilde{C}_{\Lambda \Xi, \Lambda \Xi_{*}}^{a_{\mu}^{K} \eta}=1\end{array}$ \\
\hline
\end{tabular}

$$
\begin{aligned}
& \bar{K}_{L \in[8] Q}^{(L Q), n=1}=-m_{Q}^{2}+O\left(Q^{4}\right), \\
& \frac{1}{M_{B}} \bar{K}_{L \in[8] Q}^{(L Q), n=2}=\frac{2}{3} m_{Q}^{2}+O\left(Q^{4}\right), \\
& \bar{K}_{L \in[8] Q}^{(L Q),}{ }^{n=3,5}=O\left(Q^{4}\right), \\
& \frac{1}{M_{B}} \bar{K}_{L \in[8] Q}^{(L Q), n=4}=\frac{4}{3} m_{Q}^{2}+O\left(Q^{4}\right), \\
& \bar{K}_{L \in[8] Q R \in[8]}^{(L Q), 1}=\frac{1}{2}\left(\left(\delta_{L}+\delta_{R}\right) M_{B}-m_{Q}^{2}\right)+O\left(Q^{4}\right), \\
& \bar{K}_{L \in[8] Q R \in[10]}^{(L Q), 1}=-\frac{5}{48} \frac{\Delta}{M} \alpha_{11} t-\frac{5}{36} \alpha_{12} m_{Q}^{2}-\frac{5}{12} \alpha_{14} \delta_{L} M_{B}-\frac{5}{12} \alpha_{13} \delta_{R} M_{B}+0 \alpha_{15} \delta_{B} M_{B}+O\left(Q^{4}\right), \\
& \frac{1}{M_{B}} \bar{K}_{L \in[8] Q R \in[10]}^{(L Q), 2}=\frac{1}{6} \frac{\Delta}{M} \alpha_{91} t-\frac{2}{3} \alpha_{92} m_{Q}^{2}+\frac{2}{3} \alpha_{94} \delta_{L} M_{B}-\frac{2}{3} \alpha_{93} \delta_{R} M_{B}-\frac{3}{4} \frac{\Delta}{M} \alpha_{95} \delta_{B} M_{B}+O\left(Q^{4}\right), \\
& \bar{K}_{L \in[10] Q R \in[8]}^{(L Q), 1}=-\frac{5}{48} \frac{\Delta}{M} \alpha_{21} t+\frac{19}{36} \alpha_{22} m_{Q}^{2}-\frac{5}{12} \alpha_{23} \delta_{L} M_{B}-\frac{5}{12} \alpha_{24} \delta_{R} M_{B}-\frac{5}{12} \frac{\Delta}{M} \alpha_{25} \delta_{B} M_{B}+O\left(Q^{4}\right), \\
& \frac{1}{M_{B}} \bar{K}_{L \in[10] Q R \in[8]}^{(L Q), 2}=\frac{1}{6} \frac{\Delta}{M} \alpha_{101} t+\frac{2}{3} \alpha_{102} m_{Q}^{2}+\frac{2}{3} \alpha_{103} \delta_{L} M_{B}-\frac{2}{3} \alpha_{104} \delta_{R} M_{B}+\frac{3}{4} \frac{\Delta}{M} \alpha_{105} \delta_{B} M_{B}+O\left(Q^{4}\right), \\
& \bar{K}_{L \in[10] Q R \in[10]}^{(L Q), 1}=-\frac{1}{36} \frac{\Delta}{M} \alpha_{31} t-\frac{1}{54} \alpha_{32} m_{Q}^{2}-\frac{1}{18} \alpha_{33} \delta_{L} M_{B}+\frac{5}{6} \alpha_{34} \delta_{R} M_{B}-\frac{1}{18} \frac{\Delta}{M} \alpha_{35} \delta_{B} M_{B}+O\left(Q^{4}\right), \\
& \frac{1}{M_{B}} \bar{K}_{L \in[10] Q}^{(L Q), n=1}=0 \alpha_{41} t+\frac{20}{9} \alpha_{42} m_{Q}^{2}-\frac{40}{9} \frac{\Delta}{M} \alpha_{43} \delta_{L} M_{B}-\frac{10}{3} \frac{\Delta^{2}}{M^{2}} \alpha_{45} \delta_{B} M_{B}+O\left(Q^{4}\right), \\
& \frac{1}{M_{B}} \bar{K}_{L \in[10] Q}^{(L Q), n=2}=0 \alpha_{51} t-\frac{4}{9} \alpha_{52} m_{Q}^{2}+\frac{8}{9} \frac{\Delta}{M} \alpha_{53} \delta_{L} M_{B}+\frac{2}{3} \frac{\Delta^{2}}{M^{2}} \alpha_{55} \delta_{B} M_{B}+O\left(Q^{4}\right) \text {, } \\
& \frac{1}{M_{B}} \bar{K}_{L \in[10] Q}^{(L Q), n=3}=-\frac{8}{9} \frac{\Delta^{2}}{M^{2}} \alpha_{111} t-\frac{8}{9} \frac{\Delta^{2}}{M^{2}} \alpha_{115} \delta_{B} M_{B}+O\left(Q^{4}\right),
\end{aligned}
$$




$$
\begin{aligned}
& \bar{K}_{Q R \in[8]}^{(Q R), n=1}=m_{Q}^{2}+O\left(Q^{4}\right), \quad \frac{1}{M_{B}} \bar{K}_{Q R \in[8]}^{(Q R), n=2}=\frac{2}{3} m_{Q}^{2}+O\left(Q^{4}\right), \\
& \bar{K}_{Q R \in[8]}^{(Q R), \quad n=3,5}=O\left(Q^{4}\right), \\
& \frac{1}{M_{B}} \bar{K}_{Q R \in[8]}^{(Q R), n=4}=-\frac{4}{3} m_{Q}^{2}+O\left(Q^{4}\right), \\
& \bar{K}_{L \in[8] Q R \in[8]}^{(Q R), 1}=\frac{1}{2}\left(\left(\delta_{L}+\delta_{R}\right) M_{B}-m_{Q}^{2}\right)+O\left(Q^{4}\right), \\
& \bar{K}_{L \in[10] Q R \in[8]}^{(Q R), 1}=-\frac{5}{48} \frac{\Delta}{M} \alpha_{11} t-\frac{5}{36} \alpha_{12} m_{Q}^{2}-\frac{5}{12} \alpha_{13} \delta_{L} M_{B}-\frac{5}{12} \alpha_{14} \delta_{R} M_{B}+0 \alpha_{15} \delta_{B} M_{B}+O\left(Q^{4}\right), \\
& \frac{1}{M_{B}} \bar{K}_{L \in[10] Q R \in[8]}^{(Q R), 2}=\frac{1}{6} \frac{\Delta}{M} \alpha_{91} t-\frac{2}{3} \alpha_{92} m_{Q}^{2}-\frac{2}{3} \alpha_{93} \delta_{L} M_{B}+\frac{2}{3} \alpha_{94} \delta_{R} M_{B}+\frac{3}{4} \frac{\Delta}{M} \alpha_{95} \delta_{B} M_{B}+O\left(Q^{4}\right), \\
& \bar{K}_{L \in[8] Q R \in[10]}^{(Q R), 1}=-\frac{5}{48} \frac{\Delta}{M} \alpha_{21} t+\frac{19}{36} \alpha_{22} m_{Q}^{2}-\frac{5}{12} \alpha_{24} \delta_{L} M_{B}-\frac{5}{12} \alpha_{23} \delta_{R} M_{B}+\frac{5}{12} \frac{\Delta}{M} \alpha_{25} \delta_{B} M_{B}+O\left(Q^{4}\right), \\
& \frac{1}{M_{B}} \bar{K}_{L \in[8] Q R \in[10]}^{(Q R), 2}=\frac{1}{6} \frac{\Delta}{M} \alpha_{101} t+\frac{2}{3} \alpha_{102} m_{Q}^{2}-\frac{2}{3} \alpha_{104} \delta_{L} M_{B}+\frac{2}{3} \alpha_{103} \delta_{R} M_{B}-\frac{3}{4} \frac{\Delta}{M} \alpha_{105} \delta_{B} M_{B}+O\left(Q^{4}\right), \\
& \bar{K}_{L \in[10] Q R \in[10]}^{(Q R), 1}=-\frac{1}{36} \frac{\Delta}{M} \alpha_{31} t-\frac{1}{54} \alpha_{32} m_{Q}^{2}+\frac{5}{6} \alpha_{34} \delta_{L} M_{B}-\frac{1}{18} \alpha_{33} \delta_{R} M_{B}+\frac{1}{18} \frac{\Delta}{M} \alpha_{35} \delta_{B} M_{B}+O\left(Q^{4}\right), \\
& \frac{1}{M_{B}} \bar{K}_{Q R \in[10]}^{(Q R), n=1}=0 \alpha_{41} t+\frac{20}{9} \alpha_{42} m_{Q}^{2}-\frac{40}{9} \frac{\Delta}{M} \alpha_{43} \delta_{R} M_{B}+\frac{10}{3} \frac{\Delta^{2}}{M^{2}} \alpha_{45} \delta_{B} M_{B}+O\left(Q^{4}\right), \\
& \frac{1}{M_{B}} \bar{K}_{Q R \in[10]}^{(Q R), n=2}=0 \alpha_{51} t-\frac{4}{9} \alpha_{52} m_{Q}^{2}+\frac{8}{9} \frac{\Delta}{M} \alpha_{53} \delta_{R} M_{B}-\frac{2}{3} \frac{\Delta^{2}}{M^{2}} \alpha_{55} \delta_{B} M_{B}+O\left(Q^{4}\right), \\
& \frac{1}{M_{B}} \bar{K}_{Q R \in[10]}^{(Q R), n=3}=\frac{8}{9} \frac{\Delta^{2}}{M^{2}} \alpha_{111} t-\frac{8}{9} \frac{\Delta^{2}}{M^{2}} \alpha_{115} \delta_{B} M_{B}+O\left(Q^{4}\right), \\
& \bar{K}_{L \in[8] Q R \in[8]}^{(L Q R), 1}=\bar{K}_{L \in[8] Q R \in[8]}^{(L Q R), 1}=O\left(Q^{4}\right), \\
& \frac{1}{M_{B}^{2}} \bar{K}_{L \in[10] Q R \in[8]}^{(L Q R), 1}=\frac{5}{24} \frac{\Delta^{2}}{M^{2}} \alpha_{61} t-2 \alpha_{62} m_{Q}^{2}+\frac{5}{3} \frac{\Delta}{M} \alpha_{63} \delta_{L} M_{B}+\frac{1}{3} \frac{\Delta}{M} \alpha_{64} \delta_{R} M_{B}+\frac{5}{6} \frac{\Delta^{2}}{M^{2}} \alpha_{65} \delta_{B} M_{B}+O\left(Q^{4}\right), \\
& \frac{1}{M_{B}^{3}} \bar{K}_{L \in[10] Q R \in[8]}^{(L Q R), 2}=-\frac{1}{3} \frac{\Delta^{2}}{M^{2}} \alpha_{121} t+\frac{4}{3} \frac{\Delta}{M} \alpha_{122} m_{Q}^{2}+\frac{8}{3} \frac{\Delta}{M} \alpha_{123} \delta_{L} M_{B}-\frac{8}{3} \frac{\Delta}{M} \alpha_{124} \delta_{R} M_{B}-\frac{1}{6} \frac{\Delta^{2}}{M^{2}} \alpha_{125} \delta_{B} M_{B}+O\left(Q^{4}\right), \\
& \frac{1}{M_{B}^{4}} \bar{K}_{L \in[10] Q R \in[8]}^{\prime(L Q R), 1}=0 \alpha_{71} t-\frac{2}{3} \frac{\Delta^{2}}{M^{2}} \alpha_{72} m_{Q}^{2}+\frac{4}{3} \frac{\Delta}{M} \alpha_{73} \delta_{L} M_{B}-\frac{4}{3} \frac{\Delta}{M} \alpha_{74} \delta_{R} M_{B}+\frac{2}{3} \frac{\Delta^{2}}{M^{2}} \alpha_{75} \delta_{B} M_{B}+O\left(Q^{4}\right), \\
& \frac{1}{M_{B}^{2}} \bar{K}_{L \in[8] Q R \in[10]}^{(L Q R), 1}=\frac{5}{24} \frac{\Delta^{2}}{M^{2}} \alpha_{61} t-2 \alpha_{62} m_{Q}^{2}+\frac{1}{3} \frac{\Delta}{M} \alpha_{64} \delta_{L} M_{B}+\frac{5}{3} \frac{\Delta}{M} \alpha_{63} \delta_{R} M_{B}-\frac{5}{6} \frac{\Delta^{2}}{M^{2}} \alpha_{65} \delta_{B} M_{B}+O\left(Q^{4}\right), \\
& \frac{1}{M_{B}^{3}} \bar{K}_{L \in[8] Q R \in[10]}^{(L Q R), 2}=-\frac{1}{3} \frac{\Delta^{2}}{M^{2}} \alpha_{121} t+\frac{4}{3} \frac{\Delta}{M} \alpha_{122} m_{Q}^{2}-\frac{8}{3} \frac{\Delta}{M} \alpha_{124} \delta_{L} M_{B}+\frac{8}{3} \frac{\Delta}{M} \alpha_{123} \delta_{R} M_{B}+\frac{1}{6} \frac{\Delta^{2}}{M^{2}} \alpha_{125} \delta_{B} M_{B}+O\left(Q^{4}\right), \\
& \frac{1}{M_{B}^{4}} \bar{K}_{L \in[8] Q R \in[10]}^{\prime(L Q R), 1}=0 \alpha_{71} t-\frac{2}{3} \frac{\Delta^{2}}{M^{2}} \alpha_{72} m_{Q}^{2}-\frac{4}{3} \frac{\Delta}{M} \alpha_{74} \delta_{L} M_{B}+\frac{4}{3} \frac{\Delta}{M} \alpha_{73} \delta_{R} M_{B}-\frac{2}{3} \frac{\Delta^{2}}{M^{2}} \alpha_{75} \delta_{B} M_{B}+O\left(Q^{4}\right), \\
& \frac{1}{M_{B}^{5}} \bar{K}_{L \in[10] Q R \in[8]}^{\prime(L Q R), 2}=-\frac{2}{3} \frac{\Delta^{2}}{M^{2}} \alpha_{135} \delta_{B} M_{B}+O\left(Q^{4}\right), \\
& \frac{1}{M_{B}^{5}} \bar{K}_{L \in[8] Q R \in[10]}^{\prime(L Q R), 2}=\frac{2}{3} \frac{\Delta^{2}}{M^{2}} \alpha_{135} \delta_{B} M_{B}+O\left(Q^{4}\right), \\
& \frac{1}{M_{B}^{2}} \bar{K}_{L \in[10] Q R \in[10]}^{(L Q R), 1}=-\frac{7}{9} \frac{\Delta^{2}}{M^{2}} \alpha_{81} t+\frac{4}{3} \alpha_{82} m_{Q}^{2}-\frac{4}{3} \frac{\Delta}{M} \alpha_{83} \delta_{L} M_{B}-\frac{4}{3} \frac{\Delta}{M} \alpha_{84} \delta_{R} M_{B}+0 \alpha_{85} \delta_{B} M_{B}+O\left(Q^{4}\right), \\
& \bar{K}_{L \in[10] Q R \in[10]}^{\prime(L Q R), 1}=O\left(Q^{4}\right) .
\end{aligned}
$$


The coefficients $\alpha_{i j}$, all functions of $\Delta / M$ only, are given in Eq. (F5). They are normalized to 1 in the limit $\Delta \rightarrow 0$. Power-counting violating contributions are subtracted, denoted by $\alpha_{0 j} \rightarrow 0$ and $\alpha_{j 0} \rightarrow 0$. All other factors $\alpha_{i j}$ with $i, j \neq 0$ fully enter our calculations, no expansion in $\Delta / M$ is applied:

$$
\begin{aligned}
r & =\Delta / M, \\
\alpha_{01} & =\left(12+26 r+18 r^{2}+6 r^{3}+r^{4}\right) /\left(12(1+r)^{2}\right), \\
\alpha_{02} & =(2+r)^{2}\left(6+13 r+18 r^{2}+12 r^{3}+2 r^{4}\right) /\left(24(1+r)^{4}\right), \\
\alpha_{03} & =(2+r)^{3}(5+r) /\left(40(1+r)^{2}\right), \\
\alpha_{04} & =-(-1+r)(2+r)^{3} /\left(8(1+r)^{2}\right), \\
\alpha_{10} & =(2+r)^{2}\left(5+5 r+r^{2}\right) /\left(20(1+r)^{2}\right), \\
\alpha_{11} & =(2+r)^{2}\left(5+5 r+r^{2}\right) /\left(20(1+r)^{2}\right), \\
\alpha_{12} & =-\left(-20+66 r+66 r^{2}+9 r^{3}\right) /\left(20(1+r)^{2}\right), \\
\alpha_{13} & =\left(20+60 r+87 r^{2}+65 r^{3}+23 r^{4}+3 r^{5}\right) /\left(20(1+r)^{3}\right), \\
\alpha_{14} & =(2+r)^{3}\left(5+5 r+r^{2}\right) /\left(40(1+r)^{2}\right), \\
\alpha_{15} & =0 \\
\alpha_{20} & =(2+r)^{2}\left(15+31 r+19 r^{2}+4 r^{3}\right) /\left(60(1+r)^{2}\right), \\
\alpha_{21} & =(2+r)^{2}\left(5+5 r+r^{2}\right) /\left(20(1+r)^{2}\right), \\
\alpha_{22} & =\left(76+170 r+170 r^{2}+73 r^{3}+12 r^{4}\right) /\left(76(1+r)^{2}\right), \\
\alpha_{23} & =\left(60+308 r+645 r^{2}+707 r^{3}+421 r^{4}+129 r^{5}+16 r^{6}\right) /\left(60(1+r)^{3}\right), \\
\alpha_{24} & =(2+r)^{3}\left(5+5 r+r^{2}\right) /\left(40(1+r)^{2}\right), \\
\alpha_{25} & =(2+r)^{2}\left(15+31 r+19 r^{2}+4 r^{3}\right) /\left(60(1+r)^{2}\right), \\
\alpha_{30} & =(2+r)^{3}\left(42+106 r+129 r^{2}+72 r^{3}+11 r^{4}\right) /\left(336(1+r)^{4}\right), \\
\alpha_{31} & =(2+r)^{3}\left(6+114 r+163 r^{2}+48 r^{3}+5 r^{4}\right) /\left(48(1+r)^{4}\right), \\
\alpha_{32} & =(2+r)\left(4+268 r+538 r^{2}+449 r^{3}+188 r^{4}+29 r^{5}\right) /\left(8(1+r)^{4}\right), \\
\alpha_{33} & =-(2+r)^{2}\left(-6+106 r+574 r^{2}+949 r^{3}+744 r^{4}+291 r^{5}+42 r^{6}\right) /\left(24(1+r)^{5}\right), \\
\alpha_{34} & =(2+r)^{2}\left(90+234 r+306 r^{2}+249 r^{3}+128 r^{4}+35 r^{5}+2 r^{6}\right) /\left(360(1+r)^{5}\right), \\
\alpha_{35} & =-(2+r)^{3}\left(-6-2 r+37 r^{2}+72 r^{3}+19 r^{4}\right) /\left(48(1+r)^{4}\right), \\
\alpha_{50} & =(2+r)^{4}(5+r) /\left(80(1+r)^{2}\right), \\
\alpha_{52} & \left.=-(2+r)^{2}\right) \\
\alpha_{41} & =0 \\
\alpha_{42} & =(2+r)^{2}\left(20+24 r+19 r^{2}+3 r^{3}\right) /\left(80(1+r)^{2}\right), \\
\alpha_{43} & =(2+r)^{3}\left(20+36 r+29 r^{2}+5 r^{3}\right) /\left(160(1+r)^{3}\right), \\
\alpha_{44} & =0 \\
\alpha_{50} & =(2+r)^{4}(5+r) /\left(80(1+r)^{2}\right), \\
& =\left(16(1+r)^{2}\right), \\
& =
\end{aligned}
$$




$$
\begin{aligned}
& \alpha_{53}=-(2+r)^{3}\left(-4+5 r^{2}+5 r^{3}\right) /\left(32(1+r)^{3}\right), \\
& \alpha_{54}=0 \text {, } \\
& \alpha_{55}=-(-1+r)(2+r)^{4} /\left(16(1+r)^{2}\right) \text {, } \\
& \alpha_{60}=(2+r)^{3}\left(5+5 r+r^{2}\right) /\left(40(1+r)^{2}\right) \text {, } \\
& \alpha_{61}=(2+r)^{3}\left(5+5 r+r^{2}\right) /\left(40(1+r)^{2}\right) \text {, } \\
& \alpha_{62}=(2+r)\left(48+88 r+76 r^{2}+28 r^{3}+5 r^{4}\right) /\left(96(1+r)^{2}\right) \text {, } \\
& \alpha_{63}=(2+r)^{2}\left(20+55 r+63 r^{2}+31 r^{3}+5 r^{4}\right) /\left(80(1+r)^{3}\right) \text {, } \\
& \alpha_{64}=(2+r)^{2}\left(8+28 r+33 r^{2}+9 r^{3}+r^{4}\right) /\left(32(1+r)^{2}\right) \text {, } \\
& \alpha_{65}=(2+r)^{3}\left(5+5 r+r^{2}\right) /\left(40(1+r)^{2}\right) \text {, } \\
& \alpha_{70}=(2+r)^{3}\left(1+r+r^{2}\right) /\left(8(1+r)^{2}\right) \text {, } \\
& \alpha_{71}=0 \text {, } \\
& \alpha_{72}=(2+r)^{3} /\left(8(1+r)^{2}\right) \text {, } \\
& \alpha_{73}=(2+r)^{2}\left(4+11 r+19 r^{2}+15 r^{3}+5 r^{4}\right) /\left(16(1+r)^{3}\right) \text {, } \\
& \alpha_{74}=-(2+r)^{2}\left(-8-4 r-3 r^{2}+5 r^{3}+r^{4}\right) /\left(32(1+r)^{2}\right) \text {, } \\
& \alpha_{75}=(2+r)^{3}\left(1+r+r^{2}\right) /\left(8(1+r)^{2}\right) \text {, } \\
& \alpha_{80}=(2+r)^{4}\left(6+6 r+r^{2}\right) /\left(96(1+r)^{2}\right) \text {, } \\
& \alpha_{81}=(2+r)^{4}\left(14+10 r+7 r^{2}+8 r^{3}+r^{4}\right) /\left(224(1+r)^{4}\right) \text {, } \\
& \alpha_{82}=(2+r)^{2}\left(12+24 r+25 r^{2}+13 r^{3}+2 r^{4}\right) /\left(48(1+r)^{2}\right) \text {, } \\
& \alpha_{83}=(2+r)^{3}\left(12+36 r+43 r^{2}+21 r^{3}+3 r^{4}\right) /\left(96(1+r)^{3}\right) \text {, } \\
& \alpha_{84}=(2+r)^{3}\left(12+36 r+43 r^{2}+21 r^{3}+3 r^{4}\right) /\left(96(1+r)^{3}\right) \text {, } \\
& \alpha_{85}=0 \text {, } \\
& \alpha_{90}=(2+r)^{2} /\left(4(1+r)^{2}\right) \text {, } \\
& \alpha_{91}=(2+r)^{2} /\left(4(1+r)^{2}\right) \text {, } \\
& \alpha_{92}=\left(4+3 r+r^{2}\right) /\left(4(1+r)^{2}\right) \text {, } \\
& \alpha_{93}=\left(4+4 r+3 r^{2}+r^{3}\right) /\left(4(1+r)^{3}\right) \text {, } \\
& \alpha_{94}=\left(8+10 r+11 r^{2}+6 r^{3}+r^{4}\right) /\left(8(1+r)^{2}\right), \\
& \alpha_{95}=(2+r)^{2}(9+2 r) /\left(36(1+r)^{2}\right) \text {, } \\
& \alpha_{100}=(2+r)^{2} /\left(4(1+r)^{2}\right) \text {, } \\
& \alpha_{101}=(2+r)^{2}\left(3+16 r+16 r^{2}+4 r^{3}\right) /\left(12(1+r)^{2}\right), \\
& \alpha_{102}=\left(4+3 r+r^{2}\right) /\left(4(1+r)^{2}\right) \text {, } \\
& \alpha_{103}=\left(4+4 r+3 r^{2}+r^{3}\right) /\left(4(1+r)^{3}\right) \text {, } \\
& \alpha_{104}=\left(8+10 r+11 r^{2}+6 r^{3}+r^{4}\right) /\left(8(1+r)^{2}\right) \text {, } \\
& \alpha_{105}=(2+r)^{2}\left(27+38 r+32 r^{2}+8 r^{3}\right) /\left(108(1+r)^{2}\right) \text {, } \\
& \alpha_{110}=0 \text {, } \\
& \alpha_{111}=(2+r)^{4} /\left(16(1+r)^{2}\right) \text {, } \\
& \alpha_{112}=0 \text {, }
\end{aligned}
$$




$$
\begin{aligned}
& \alpha_{113}=0, \\
& \alpha_{114}=0, \\
& \alpha_{115}=(2+r)^{4} /\left(16(1+r)^{2}\right), \\
& \alpha_{120}=(2+r)^{3} /\left(8(1+r)^{2}\right), \\
& \alpha_{121}=(2+r)^{3} /\left(8(1+r)^{2}\right), \\
& \alpha_{122}=(2+r)\left(4+3 r+r^{2}\right) /\left(8(1+r)^{2}\right), \\
& \alpha_{123}=(2+r)^{2}\left(4+5 r+3 r^{2}\right) /\left(16(1+r)^{3}\right), \\
& \alpha_{124}=(2+r)^{2}\left(8+3 r+4 r^{2}+r^{3}\right) /\left(32(1+r)^{2}\right), \\
& \alpha_{125}=(2+r)^{3}(1+2 r) /\left(8(1+r)^{2}\right), \\
& \alpha_{130}=0, \\
& \alpha_{131}=0, \\
& \alpha_{132}=0, \\
& \alpha_{133}=0, \\
& \alpha_{134}=0, \\
& \alpha_{135}=(2+r)^{3}(1+2 r) /\left(8(1+r)^{2}\right) .
\end{aligned}
$$

Note that all $\alpha_{i j}$ are the same as in Ref. [5]. The only exception is $\alpha_{33}$ of Ref. [5], which is given by

$$
-\frac{1}{14} \alpha_{33}+\frac{15}{14} \alpha_{34}
$$

[1] N. Cabibbo, Phys. Rev. Lett. 10, 531 (1963).

[2] N. Cabibbo, E. C. Swallow, and R. Winston, Annu. Rev. Nucl. Part. Sci. 53, 39 (2003).

[3] E. Jenkins and A. V. Manohar, Phys. Lett. B 255, 558 (1991).

[4] E. Jenkins and A. V. Manohar, Phys. Lett. B 259, 353 (1991).

[5] M. F. Lutz, U. Sauerwein, and R. G. Timmermans, Eur. Phys. J. C 80, 844 (2020).

[6] R. Gupta, Y.-C. Jang, H.-W. Lin, B. Yoon, and T. Bhattacharya, Phys. Rev. D 96, 114503 (2017).

[7] S. Capitani, M. Della Morte, D. Djukanovic, G. M. von Hippel, J. Hua, B. Jäger, P. M. Junnarkar, H. B. Meyer, T. D. Rae, and H. Wittig, Int. J. Mod. Phys. A 34, 1950009 (2019).

[8] C. Alexandrou, M. Constantinou, K. Hadjiyiannakou, K. Jansen, C. Kallidonis, G. Koutsou, and A. Vaquero AvilesCasco, Phys. Rev. D 96, 054507 (2017).

[9] G. S. Bali, S. Collins, M. Gruber, A. Schäfer, P. Wein, and T. Wurm, Phys. Lett. B 789, 666 (2019).

[10] A. Semke and M. F. Lutz, Nucl. Phys. A789, 251 (2007).
[11] X.-Y. Guo, Y. Heo, and M. F. Lutz, Eur. Phys. J. C 80, 260 (2020).

[12] K.-I. Ishikawa, Y. Kuramashi, S. Sasaki, N. Tsukamoto, A. Ukawa, and T. Yamazaki (PACS Collaboration), Phys. Rev. D 98, 074510 (2018).

[13] C. Alexandrou et al., Proc. Sci., LATTICE2018 (2018) 142 [arXiv:2112.06750].

[14] G. S. Bali, L. Barca, S. Collins, M. Gruber, M. Löffler, A. Schäfer, W. Söldner, P. Wein, S. Weishäupl, and T. Wurm (RQCD Collaboration), J. High Energy Phys. 05 (2020) 126.

[15] G. S. Bali et al., Proc. Sci., LATTICE2019 (2019) 099 [arXiv:1907.13454].

[16] M. Lutz and E. Kolomeitsev, Nucl. Phys. A700, 193 (2002).

[17] M. F. M. Lutz and A. Semke, Phys. Rev. D 83, 034008 (2011).

[18] M. Frink and U.-G. Meissner, Eur. Phys. J. A 29, 255 (2006).

[19] S. Aoki et al. (Flavour Lattice Averaging Group), Eur. Phys. J. C 80, 113 (2020). 
[20] J. A. Oller, M. Verbeni, and J. Prades, J. High Energy Phys. 09 (2006) 079.

[21] J. A. Oller, M. Verbeni, and J. Prades, arXiv:hep-ph/ 0701096.

[22] M. Holmberg and S. Leupold, Eur. Phys. J. A 54, 103 (2018).

[23] A. Semke and M. Lutz, Nucl. Phys. A778, 153 (2006).

[24] M. Tanabashi et al. (Particle Data Group), Phys. Rev. D 98 , 030001 (2018).

[25] H.-W. Lin and K. Orginos, Phys. Rev. D 79, 034507 (2009).

[26] M. Gockeler et al. (QCDSF/UKQCD Collaborations), Proc. Sci., LATTICE2010 (2010) 163 [arXiv:1102.3407].

[27] J. Gasser and H. Leutwyler, Nucl. Phys. B250, 465 (1985).

[28] G. 't Hooft and M. Veltman, Nucl. Phys. B44, 189 (1972).
[29] G. Leibbrandt, Rev. Mod. Phys. 47, 849 (1975).

[30] T. Becher and H. Leutwyler, Eur. Phys. J. C 9, 643 (1999).

[31] T. R. Hemmert, B. R. Holstein, and J. Kambor, J. Phys. G Nucl. Part. Phys. 24, 1831 (1998).

[32] M. Lutz, Y. Heo, and X.-Y. Guo, Nucl. Phys. A977, 146 (2018).

[33] T. Ledwig, J. M. Camalich, L. S. Geng, and M. J. V. Vacas, Phys. Rev. D 90, 054502 (2014).

[34] V. Bernard, D. Hoja, U.-G. Meißner, and A. Rusetsky, J. High Energy Phys. 06 (2009) 061.

[35] X.-L. Ren, L. Geng, J. Meng, and H. Toki, Phys. Rev. D 87, 074001 (2013).

[36] B. Long and U. van Kolck, Nucl. Phys. A840, 39 (2010).

[37] W. Bietenholz et al., Phys. Rev. D 84, 054509 (2011).

[38] H. Haberzettl, arXiv:nucl-th/9812043. 